\title{
Axonal degeneration and protection during early remyelination in multiple sclerosis and an animal model
}

\section{Doctoral Thesis}

In partial fulfillment of the requirements for the degree

"Doctor rerum naturalium (Dr. rer. nat.)"

in the Molecular Medicine Study Program

at the Georg-August-University Göttingen

\author{
submitted by \\ Verena Schultz \\ born in \\ Wilhelmshaven
}

Göttingen, November 2014 


\section{Members of the Thesis Committee:}

\section{Supervisor}

Prof. Dr. Wolfgang Brück

Department of Neuropathology

University Medical Centre

Georg-August-University, Göttingen

Second member of the thesis committee

Prof. Dr. Thomas Bayer

Department for Psychiatry

Division of Molecular Psychiatry, Göttingen

Third member of the thesis committee

Dr. Till Marquardt

European Neuroscience Institute Göttingen

Developmental Neurobiology Group, Göttingen

Date of Disputation: January $22^{\text {nd }}, 2014$ 
FOR MY SISTER MARIANNE. 


\section{AFFIDAVIT}

Here I declare that my doctoral thesis entitled "Axonal degeneration and protection during early remyelination in multiple sclerosis and an animal model" has been written independently with no other sources and aids than quoted.

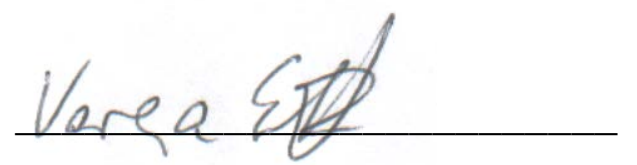

Verena Schultz

Göttingen, November 2014 


\section{RELATED PUBLICATIONS}

\section{Original articles}

Lescher J, Paap F, Schultz V, Redenbach L, Scheidt U, Rosewich H, Nessler S, Fuchs E, Gärtner J, Brück W, Junker A. (2012) MicroRNA regulation in experimental autoimmune encephalomyelitis in mice and marmosets resembles regulation in human multiple sclerosis lesions. J. Neuroimmunol. 246, 27-33.

\section{Abstracts/ Presentations}

Schultz V, Paap F, Scheidt U, Brück W, Junker A. Axonal degeneration and protection during early remyelination in multiple sclerosis and an animal model. Presented as a poster at the 58th Annual meeting of the German Society for Neuropathology and Neuroanatomy (DGNN), 11th-14th September 2012, in Göttingen, Germany

Schultz V, Paap F, Scheidt U, Junker A. Disrupted CD47 signaling in MS lesions leads to failed remyelination. Presented as a talk at the 5th NEUROWIND e.V. meeting, 25th-27th October 2013, in Berlin, Germany

Schultz V, Paap F, Scheidt U, Stadelmann C, Brück W, Junker A. Axonal degeneration and protection during early remyelination in multiple sclerosis and an animal model. Presented as a poster at the 12th International Congress of Neuroimmunology (ISNI), 09th-13th November 2014, in Mainz, Germany 


\section{TABLE OF CONTENTS}

Acknowledgements

Abstract. III

List of Figures

List of Tables VII

Abbreviations. VIII

1 Introduction ......................................................................... 1

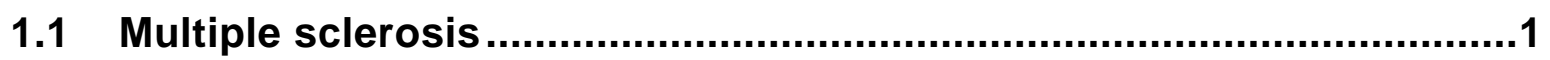

1.1.1 Clinical characteristics of multiple sclerosis .................................... 1

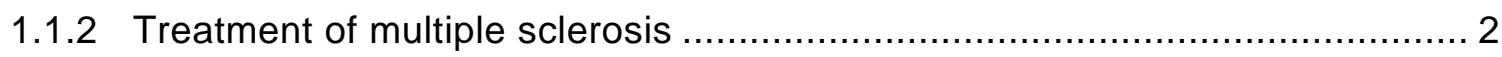

1.1.2.1 The mechanisms of action of 4-aminopyridine and amiloride ................ 3

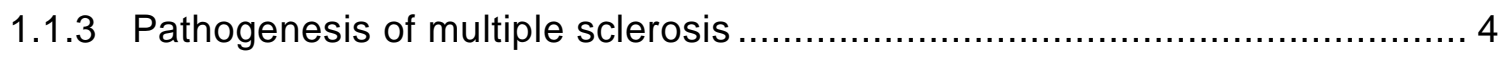

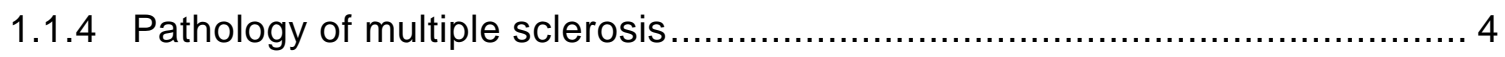

1.2 Cuprizone mouse model............................................................5

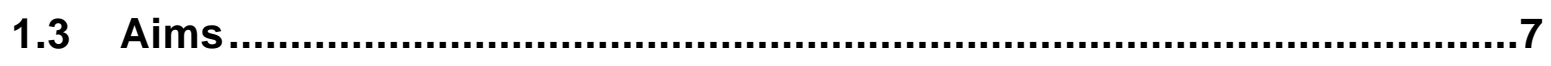

2 Materials and Methods ..................................................... 8

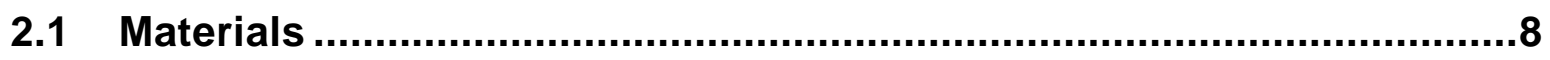

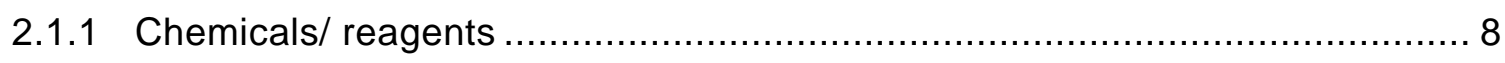

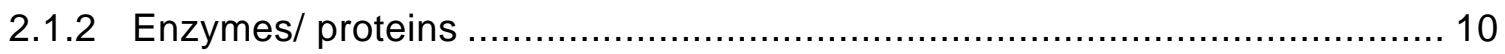

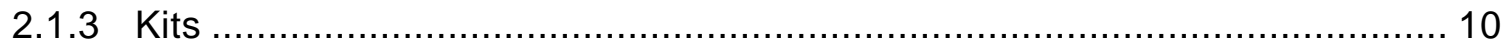

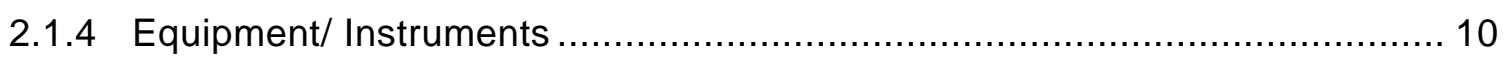

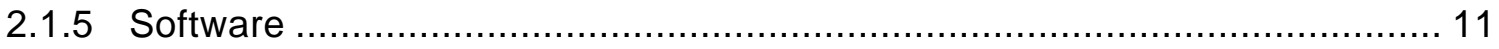


2.2 Methods ................................................................................... 12

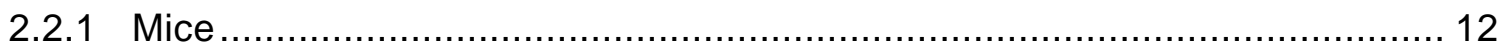

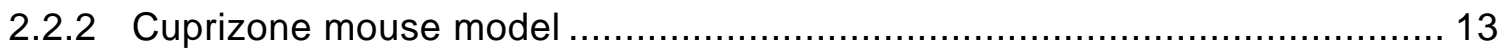

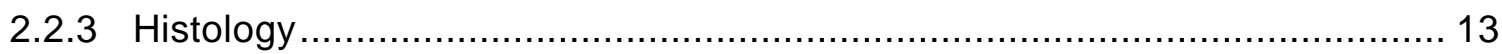

2.2.3.1 Luxol fast blue-periodic acid Schiff (LFB-PAS) staining …................. 14

2.2.3.2 Immunohistochemistry and fluorescence immunohistochemistry .......... 14

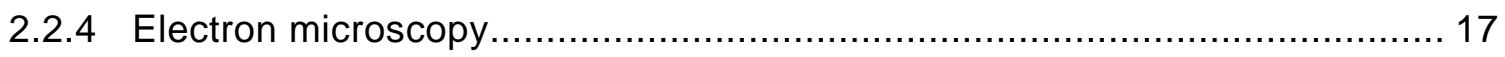

2.2.5 Morphometry and data aquisition .................................................... 17

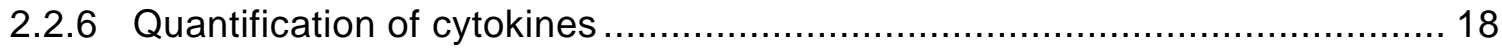

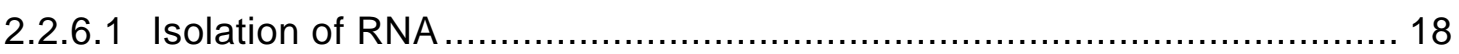

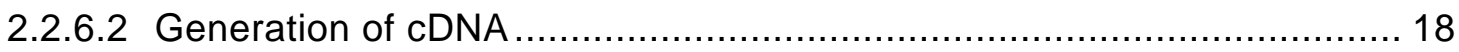

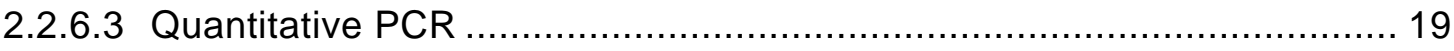

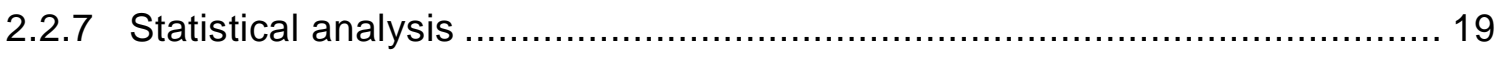

3 Results............................................................................ 21

3.1 Investigation of acute axonal damage during early remyelination in the cuprizone mouse model and in MS lesions

3.1.1 Efficient regeneration of the corpus callosum after cuprizone diet cessation

3.1.2 Acute axonal damage was detectable during early and late

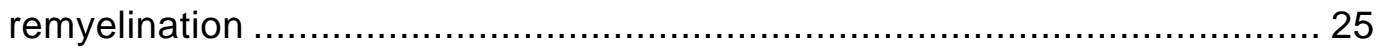

3.1.3 Microgliosis was declining during remyelination while astrogliosis sustained over time

3.1.4 Expression of TGF $\beta$ and TNF $\alpha$ was upregulated during demyelination and remyelination

3.1.5 The percentage of myelinated damaged axons increased during remyelination

3.1.6 The corpus callosum exhibited large demyelinated areas without intact myelin sheaths after cuprizone challenge 
3.1.7 (Re)myelinated damaged axons were observed in early remyelinating early active MS lesions

3.1.8 Late remyelinated MS lesions exhibited less acutely damaged axons than chronic inactive MS lesions

3.1.9 Colocalization of the paranodal protein Caspr with $\mathrm{APP}^{+}$spheroids in the cuprizone mouse model

3.1.10 Cell processes of reactive astrocytes were found in the proximity of damaged axons

\subsection{Effects of ion channel inhibition on acute axonal damage during} demyelination and remyelination

3.2.1 Acute axonal damage was not influenced by $K_{V}$ channel inhibition during remyelination.

3.2.2 $\mathrm{K}_{\mathrm{V}}$ channel inhibition did not reduce significantly the percentage of myelinated damaged axons

3.2.3 The number of reactive astrocytes was not influenced by $\mathrm{K}_{\mathrm{V}}$ channel inhibition

3.2.4 Microgliosis was increased during remyelination by $\mathrm{K}_{v}$ channel inhibition.... 39

3.2.5 The number of oligodendrocytes was accelerated in the medial corpus callosum by $\mathrm{K}_{\mathrm{v}}$ channel inhibition during remyelination.

3.2.6 ASIC inhibition did not modify acute axonal damage or astrogliosis, but modified microgliosis

3.2.7 The number of oligodendrocytes was not influenced by ASIC inhibition

3.2.8 Inhibition of $\mathrm{K}_{\mathrm{V}}$ channels did not alter the demyelinated area of the corpus callosum in the cuprizone mouse model

4 Discussion

4.1 Acute axonal damage in relation to early remyelination

4.1.1 Efficient regeneration of the corpus callosum after cuprizone diet cessation

4.1.1.1 The cuprizone mouse model to study remyelination 49

4.1.1.2 Oligodendrocytes in the cuprizone mouse model 49 
4.1.2 Acute axonal damage in the cuprizone mouse model

4.1.2.1 Acute axonal damage detectable in the corpus callosum after six weeks of cuprizone treatment

4.1.2.2 Acute axonal damage decreased during remyelination

4.1.3 Decreased activated microglia and sustained reactive astrocytes during remyelination in the cuprizone mouse model

4.1.3.1 Activated microglia during remyelination

4.1.3.2 Reactive astrocytes during remyelination

4.1.4 Expression of TGF $\beta$ and TNF $\alpha$ was upregulated during demyelination and remyelination

4.1.5 The subpopulation of myelinated damaged axons increased during remyelination

4.1.6 Contiguous large demyelinated areas without intact myelin sheaths in the corpus callosum after cuprizone challenge

4.1.7 (Re)myelinated damaged axons were also present in early remyelinating early active MS lesions

4.1.8 Remyelinated MS lesions exhibited less acute damaged axons than chronic inactive MS lesions.

4.1.9 Colocalization of the paranodal protein Caspr with $\mathrm{APP}^{+}$spheroids in the cuprizone mouse model

4.1.10 Conclusion.

\subsection{Ion channel blockage during demyelinating and remyelinating} processes in the CNS

4.2.1 Axonal pathology during demyelination and remyelination in mice treated with ion channel inhibitors-potentially beneficial?

4.2.2 Increased numbers of microglial cells during remyelination by ion channel inhibition, but unaltered reactive astrogliosis

4.2.3 Demyelination and remyelination in the cuprizone mouse modelbeneficial effects by ion channel inhibition

4.2.4 Conclusion. 


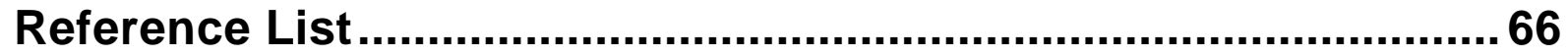

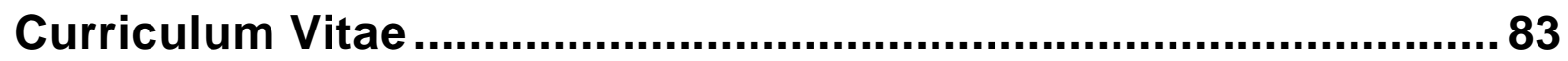




\section{ACKNOWLEDGEMENTS}

I would like to acknowledge my supervisor Prof. Dr. Wolfgang Brück for giving me the opportunity to join the department of neuropathology in Göttingen. I would like to thank him for supervising my project and for his scientific discussions. I would also like to acknowledge Dr. Andreas Junker for his scientific advices and his excellent support during my PhD years. I am grateful for his scientific and innovative discussions. I would like to express gratitude to Prof. Dr. Christine Stadelmann-Nessler for sharing her scientific expertise, for her patience and for her helpful advices.

I acknowledge Prof. Dr. Thomas Bayer for his comments and the helpful and supporting discussions. I am grateful for his supportive contribution in project discussions as the second member of my thesis committee. I want to thank Dr. Till Marquardt for his interest in my project and for his comments and ideas in project discussions as the third member of my thesis committee.

I sincerely thank Prof. Christopher Linington for giving me the opportunity to join his lab for an internship and for his scientific discussions. I want to thank Dr. Maren Lindner for sharing her scientific knowledge and for her supportive comments and ideas on cell culture techniques and mouse models.

I acknowledge Franziska Paap for her technical support and discussions on RNAisolation and GPCR. I want to thank her for her helpful discussions and proofreading of my PhD thesis. I am grateful for her honesty and her support during the daily routine in the lab.

I want to thank Xenia Schulz for her helpful discussions and her supportive comments on statistical issues.

I would like to thank the laboratory assistants Uta, Brigitte, and Doris for their theoretical discussions and practical support on histology and immunohistochemistry. I am thankful for the nice conversations and the enjoyable atmosphere in the lab.

I want to thank Sarah, Franziska, Insa, Hana, Anne, Nasrin, Christin, Ulla, Martina, Shailender... and all other colleagues for the great time in the neuropathology 
in Göttingen. I owe you many thanks for the evenings we spent together and the funny time we had inside and outside the lab.

I would like to acknowledge the Molecular Medicine PhD program for administrative support. I want to thank our secretary Cynthia for her friendly conversations and her administrative support.

Special thanks to my parents Ingo and Ulrike and my siblings Marianne, Roswitha, Mark, Raphael and Frederick for supporting me over all these years and in every decision. Finally, I sincerely thank my sister Marianne for her poetic words and her persistent confidence in me. You make things possible. 


\section{ABSTRACT}

Axonal damage, often followed by axonal degeneration, is the main cause of persisting disabilities in multiple sclerosis (MS) patients and already occurs in early stages of the disease. Disease progression is in addition characterized by degenerative processes such as oligodendroglial death, demyelination and axonal damage/ degeneration, which seem at least in part uncoupled from inflammation. It has been demonstrated that axonal damage is most pronounced in early actively demyelinating MS lesions. Furthermore, demyelinated axons show a higher vulnerability than properly myelinated axons. Thus, remyelination and the formation of new nodes of Ranvier seem to be neuroprotective. Hence, in the present study I investigated the temporal and functional relation between myelin protein expression and acute axonal damage during remyelination in remyelinating/ remyelinated MS lesions and in the cuprizone mouse model. Furthermore, I used this model to assess the therapeutic potential of the ion channel blockers 4-aminopyridine (4-AP) and amiloride with regard to protection from axonal damage.

C57BI/6 mice were treated for six weeks with the copper chelator cuprizone ( $0.25 \%$ in normal chow) which leads to demyelination of the brain, especially of the corpus callosum. After discontinuation of the cuprizone diet, remyelination occurs within a few days and is completed after several weeks during which the brains were harvested and analyzed. The pathology in the corpus callosum was analyzed by histopathology, quantitative PCR and electron microscopy. The corpus callosum of mice was rapidly remyelinated during the first week of cuprizone free diet. Furthermore, a reconstitution of the density of oligodendrocytes, which was comparable to untreated mice, was accomplished one week after cuprizone withdrawal. Microgliosis was significantly diminished already after two days of remyelination and declines even further thereafter, while astrogliosis persisted during remyelination. Furthermore, mice exhibited a continuous decrease of acutely damaged axons during remyelination. Remarkably, acute axonal damage that was directly associated with complete or partial myelination was observed by confocal microscopy. Over time the quantity of myelinated axonal spheroids increased significantly, which, given the strong regenerative processes observed, suggests that remyelination may occur randomly and independent of axonal functionality. Furthermore, myelinated acutely damaged axons were also seen in early remyelinating MS lesions. These results suggest that remyelination may occur independent of axonal transport disturbances in MS, however, this hypothesis has still to be substantiated in future studies. 
To investigate the influence of voltage-gated potassium $\left(\mathrm{K}_{\mathrm{V}}\right)$ channel and acid sensing ion channel (ASIC) inhibition on acute axonal damage during early remyelination, mice were treated either with 4-AP or with amiloride during demyelination and remyelination. Despite an increased microgliosis induced by these ion channel inhibitors acute axonal damage was comparable to PBS-treated controls. However, administration of 4-aminopyridine during remyelination resulted in increased numbers of oligodendrocytes. Thus, administration of 4-aminopyridine during remyelination may have beneficial effects on oligodendrocytes. The inefficacy of 4-aminopyridine and amiloride to decrease the acute axonal damage during remyelination might be a consequence of the strong regenerative processes observed during remyelination. To evaluate the axonprotective capacity of ion channel inhibition during demyelination, mice were treated with 4-aminopyridine or amiloride during acute cuprizone-induced demyelination. Both ion channel inhibitors did not significantly modify acute axonal damage, the numbers of oligodendrocytes and the demyelination in the corpus callosum of mice during cuprizone ingestion. These findings revealed that the inhibition of $K_{V}$ channels or ASICs did not significantly modify the extent of acute axonal damage during cuprizone treatment and during remyelination. 


\section{LIST OF FIGURES}

Figure 1: Design of the cuprizone experiment to investigate the acute axonal damage during early remyelination.

Figure 2: $\quad$ Remyelination as a regenerative process started within a few days after cuprizone withdrawal.

Figure 3: $\quad$ Semi quantitative analysis of myelin revealed rapid remyelination but sequential myelin protein expression of the corpus callosum, which started with CNP, followed by MBP, MOG, and PLP.

Figure 4: Numerous oligodendrocyte precursor cells were observed after six weeks of cuprizone-induced demyelination.

Figure 5: The number of OPCs and mature oligodendrocytes seemed to be reestablished after one week of recovery.

Figure 6: Acute axonal damage was detected during early and late remyelination.

Figure 7: Microgliosis was declining during remyelination while astrogliosis sustained over time.

Figure 8: Expression of TGF $\beta$ and TNF $\alpha$ was upregulated during demyelination and remyelination.

Figure 9: The percentage of myelinated damaged axons increased during remyelination.

Figure 10: Electron microscopic analysis revealed disrupted myelin sheaths and increased mitochondrial content of damaged axons.

Figure 11: $\quad$ Myelinated $\mathrm{APP}^{+}$spheroids were observed in early remyelinating early active MS lesions.

Figure 12: Late remyelinated MS lesions exhibited less $\mathrm{APP}^{+}$spheroids than chronic inactive MS lesions.

Figure 13: The paranodal protein Caspr was colocalized with $\mathrm{APP}^{+}$spheroids in the cuprizone mouse model.

Figure 14: $\mathrm{APP}^{+}$spheroids were surrounded by cell processes of reactive astrocytes in the cuprizone mouse model. 
Figure 15: Design of the cuprizone experiments to investigate the effects of ion channel inhibition on acute axonal damage during remyelination.

Figure 16: $\quad K_{V}$ channel inhibition did not influence the acute axonal damage during remyelination.

Figure 17: $\quad$ Mice treated with 4-aminopyridine exhibited no significant difference in the percentage of myelinated $\mathrm{APP}^{+}$spheroids compared to control....

Figure 18: No difference in the number of reactive astrocytes by $K_{v}$ channel inhibition during remyelination.

Figure 19: Microgliosis was increased by $K_{V}$ channel inhibition during remyelination. 40

Figure 20: The number of oligodendrocytes was accelerated in the medial corpus callosum by $\mathrm{K}_{\mathrm{v}}$ channel inhibition during remyelination.

Figure 21: ASIC inhibition did neither modify acute axonal damage nor astrogliosis.

Figure 22: ASIC inhibition exhibited a tendency to accelerate the extent of microgliosis.

Figure 23: The number of oligodendrocytes was not influenced by ASIC inhibition.

Figure 24: Acute axonal damage of 4-aminopyridine-treated and amiloridetreated mice was not significantly different compared to vehicletreated mice during demyelination.

Figure 25: The numbers of oligodendrocytes were not significantly altered in inhibitor-treated mice compared to vehicle-treated mice during demyelination.

Figure 26: $\quad$ Mice treated with 4-aminopyridine or amiloride showed no difference in demyelination in comparison to vehicle-treated mice. 


\section{LIST OF TABLES}

Table 1: List of primary antibodies for immunhistochemistry and fluorescence immunohistochemistry ............................................................ 15

Table 2: List of secondary antibodies for immunhistochemistry and fluorescence immunohistochemistry ............................................... 16

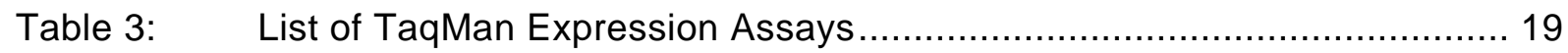




\section{ABBREVIATIONS}

$\mu g$

$\mu \mathrm{m}$

AgNO3

ANOVA

APP

ASIC

ATP

BBB

BDNF

BSA

C

$\mathrm{Ca}$

CCL2

CCL3

CIS

$\mathrm{cm}$

CNPase

CNS

CXCL10

$D A B$

DAPI

EAE

EBV

EDTA

EM

et al

FCS

FGF

GFAP

$\mathrm{h}$

$\mathrm{H} 2 \mathrm{O} 2$ microgram

micrometer

silver nitrate

analysis of variance

amyloid precursor protein

acid sensing ion channel

adenosine-5-triphosphate

blood- brain-barrier

brain-derived neurotrophic factor

bovine serum albumin

celsius

calcium

chemokine ligand 2

chemokine ligand 3

clinically isolated syndrome

centimeter

2',3'-cyclic nucleotide 3'-

phosphodiesterase

central nervous system

C-X-C motif chemokine 10

3,3'-diaminobenzidine

4',6-diamidino-2-phenylindole

experimental autoimmune

encephalomyelitis

Epstein-Barr-Virus

ethylenediamine tetraacetic acid

disodiumsalt dihydrate

electron microscopy

and others

fetal calf serum

fibroblast growth factor

glial fibrilliary acidic protein

hour

hydrogen peroxide 
HE

HLA

i.p.

IFN

IGF

$\lg G$

IHC

IL

$\mathrm{K}$

$\mathrm{kg}$

$\mathrm{K}_{\mathrm{V}}$ channel

I

LFB-PAS

LPS

$\mathrm{M}$

MAG

MBP

MHC

$\min$

$\mathrm{mm}$

MOG

MS

$\mathrm{n}$

$\mathrm{Na}$

$\mathrm{NaCl}$

$\mathrm{NaOH}$

NAWM

NogoA

Olig2

OPC

PBS

PCR

PDGF

PFA

PLP

PP
Hematoxylin Eosin

histocompatibility leukocyte antigen

intraperitoneal

Interferon

Insulin-like growth factor

Immunoglobulin G

immunohistochemistry

Interleukin

potassium

kilogram

voltage-gated potassium channel

liter

Luxol fast blue-periodic acid Schiff

Lipopolysaccharide

Molar

Myelin-associated glycoprotein

Myelin basic protein

major histocompatibility complex

minutes

millimeter

myelin oligodendrocyte glycoprotein

multiple sclerosis

number of mice

sodium

sodium chloride

sodium hydroxide

normal-appearing white matter

neurite outgrowth inhibitor $A$

oligodendrocyte transcription factor 2

oligodendrocyte precursor cell

phosphate buffered saline

polymerase chain reaction

platelet derived growth factor

paraformaldehyde

proteolipid protein

primary progressive 
$\mathrm{RR}$

$\mathrm{S}$

S.C.

SEM

SP

TGF $\beta$

Th

TNFa

Tris

v

w relapsing-remitting

seconds

subcutan

standard error of the mean

secondary progressive

transforming growth factor beta

$\mathrm{T}$ helper cell

tumor necrosis factor alpha

tris(hydroxymethyl)aminomethane

volume

weight 


\section{$1 \quad$ INTRODUCTION}

\subsection{Multiple sclerosis}

Multiple sclerosis (MS) is a chronic inflammatory disease of the central nervous system (CNS) including brain and spinal cord which is characterized by focal demyelinating lesions with partial axon preservation and glial scar formation. Despite partial preservation of axons axonal damage and degeneration nevertheless occur, resulting in chronic disabilities in early to middle adulthood of MS patients. The disease was identified as an entity by Jean Martin Charcot in 1868, who described multiple sclerosis as a focal inflammatory demyelinating disease of the white matter. However recent work on MS pathology has elucidated the important role of pathological changes in the normal-appearing white matter (NAWM) and grey matter of MS patients. The pathology is not restricted to focal lesion sites, but affects the entire CNS. Especially during the early disease phases in MS, demyelination can be partially reversed by spontaneous remyelination, which occurs erratically and insufficiently in MS patients (Kutzelnigg and Lassmann, 2014).

\subsubsection{Clinical characteristics of multiple sclerosis}

The clinical course of multiple sclerosis varies highly between patients and is thus difficult to predict. The clinical symptoms can be numerous and include numbness, tingling, weakness, vision loss, gait impairment, ataxia, imbalance or bladder dysfunction. In addition, approximately half of the MS patients report that they suffer from fatigue, a symptom which is difficult to measure clinically. The disease course is either continuous with symptoms accumulating over time (progressive MS) or consists of volatile attacks (relapsing-remitting MS). Multiple sclerosis can be differentiated into three main subtypes: relapsing-remitting MS (RRMS), secondary progressive MS (SPMS), and primary progressive MS (PPMS) (Gelfand, 2014;Kremenchutzky et al., 2006;Lublin and Reingold, 1996).

The relapsing-remitting form is the most common form of MS and affects $80-90 \%$ of patients during the first years of the disease. The attacks evolve over hours to days and persist for several days to weeks before remitting. The patients are otherwise stable, but tend to experience fatigue or heat sensitivity. Patients with RRMS often develop a secondary progressive disease course (80\% within 20 years) which is characterized by insidious neurologic worsening and accumulating disability that is not related to discrete attacks. However the progression from RRMS to SPMS appears as a continuum which sometimes occurs on the background of relapses (Gelfand, 2014;Kremenchutzky et al., 2006; Lublin and Reingold, 1996). 
$10-20 \%$ of patients are diagnosed with PPMS, i.e. they never experience a relapse and show continuous neurologic worsening and accumulating disability from the onset of symptoms on (Gelfand, 2014).

The initial stage of the clinical disease is designated as clinically isolated syndrome (CIS), which is the first clinically apparent demyelinating attack. Here, the diagnosis of MS is not possible on the basis of the currently established diagnostic criteria, the so-called "McDonald criteria". These diagnostic criteria focus on clinical and radiologic evidence of the dissemination of MS lesions in time and space (McDonald et al., 2001;Polman et al., 2011). Nevertheless, the majority (75-82\%) of the patients with CIS develop MS (Gelfand, 2014).

\subsubsection{Treatment of multiple sclerosis}

Preventive or curative therapies are thus far not available as the etiology of MS is still unknown. Nevertheless several factors could be identified which make the development of disease more probable: 1 ) the presence of susceptibility genes (HLADRB1, HLA-DRB2, HLA-DQB2; SNPs in receptors of the cytokines IL2RA, IL7RA and membrane proteins CD58 or CTLA-4) 2) the fact that the prevalence is substantially increased in family members of MS patients 3 ) the presence of infectious agents (EBV, HHV-6) and 4) environmental factors (vitamin D3 deficiency) (Goodin, 2014b).

However, several medications have been established to treat MS patients according to the clinical presentation. These can be subdivided into four main therapeutic aims: 1) to limit the severity and duration of an acute attack, 2) to reduce the biological activity of MS (= disease-modifying therapy [DMT]; e.g. Glatiramer acetate, IFN $\beta$, dimethyl fumarate, fingolimod, teriflunomide, alemtuzumab, natalizumab, mitoxantrone), 3) to improve or eliminate the various clinical symptoms (e.g. bladder dysfunction, spasticity, fatigue), 4) to promote the protection from, and the repair of, damage caused by the inflammatory process. None of the drugs available belong unequivocally in the fourth category, although DMTs appear sometimes to be neuroprotective or neuroreparative (Goodin, 2014a). Glatiramer acetate, for example, induced secretion of BDNF by T cells, which was shown to be neuroprotective in EAE (Linker et al., 2010). Another drug, which is potentially capable of indirect and direct neuroprotection, is dimethyl fumarate, which exhibits neuroprotective function by upregulating the expression of Nrf2 and thereby increasing cellular anti-oxidative mechanisms (Melzer and Meuth, 2014).

4-Aminopyridine (Fampridine), a voltage-gated potassium channel inhibitor, is a medication used for symptomatic treatment (group 3) of MS patients and was originally described for the treatment of fatigue (Toosy et al., 2014). The synaptic transmission is 
potentiated and the skeletal muscle tension is increased by administration of the drug at clinical doses. Although the results of the clinical trials are controversial, some studies showed that walking ability in MS patients improved up to $25 \%$. The drug is well tolerated, but has potential side effects: paresthesias, dizziness, lightheadedness, insomnia, anxiety, asthenia, nausea, headache and tremor. Especially at high doses of 4aminopyridine epileptic seizures may occur (Toosy et al., 2014).

Amiloride, an acid-sensing ion channel inhibitor, was recently described to be neuroprotective in a small cohort of 14 MS patients with PPMS (Arun et al., 2013). The rates of brain atrophy and tissue damage were compared during pre-treatment and amiloride administration. Significant beneficial effects during amiloride treatment were observed on the basis of MRI. However this study lacks pathological specificity of neuroprotection in MS patients (Arun et al., 2013). The drug is well tolerated and approved for treatment of hypertension and congestive heart failure (Friese et al., 2007) and might be a good candidate for tissue protection and repair (group 4).

\subsubsection{The mechanisms of action of 4-aminopyridine and amiloride}

4-Aminopyridine inhibits fast voltage-gated potassium channels in excitable tissues and non-excitable cells (Hayes, 2004). Potassium channels of axons are mainly covered by a myelin sheath in healthy conditions. Demyelination of axons causes the exposure of ion channels to the environment, which results in membrane hyperpolarization. The threshold for successful axon potential propagation is thereby increased. Furthermore, the loss of anchoring proteins leads to diffusion of potassium channels in the membrane. These changes cause abnormal potassium outward currents associated with slow action potential conduction, conduction failure or changes in the axon's capacity to discharge repetitively (Bittner and Meuth, 2013). 4-Aminopyridine is able to restore the conduction in focally demyelinated axons (Hayes, 2004). Furthermore administration of 4-aminopyridine increases calcium influx at presynaptic terminals, which enables an enhancement of neuronal or neuromuscular transmission in myelinated axons (Hayes, 2004).

Amiloride is an inhibitor of acid-sensing ion channels (ASICs) which are protongated ion channels and belonging to a subgroup of the degenerin-epithelial channel family of cation channels. These channels can flux $\mathrm{Na}^{+}$and $\mathrm{Ca}^{2+}$ ions and consist of six isoforms: ASIC1a, ASIC1b, ASIC2a, ASIC2b, ASIC3 and ASIC4. The predominant functional ion channel subunit in the CNS is ASIC1a (Bittner and Meuth, 2013;Friese et al., 2007; Vergo et al., 2011). CNS inflammation results in release of nitric oxide (NO) and free oxygen radicals by inflammatory cells such as activated microglia and macrophages, which leads to mitochondrial damage with subsequent energy failure and tissue acidosis. 
The emerging tissue acidosis activates the ASICs and $\mathrm{Na}^{+}$and $\mathrm{Ca}^{2+}$ ions accumulate intracellularly. The increased intracellular concentration of $\mathrm{Ca}^{2+}$ ions leads to an activation of secondary injury cascades such as proteases and thereby to axonal degeneration (Bittner and Meuth, 2013). The ASIC inhibitor amiloride was reported to protect the CNS against demyelination and axonal damage (Arun et al., 2013;Bittner and Meuth, 2013;Friese et al., 2007; Vergo et al., 2011).

\subsubsection{Pathogenesis of multiple sclerosis}

Animal models with experimental autoimmune encephalomyelitis (EAE) are considered to display central features of MS pathogenesis. The findings in these models suggest that MS is a predominantly $\mathrm{T}$ cell-mediated autoimmune disease and develops after activation of autoreactive $\mathrm{CD} 4^{+}$and $\mathrm{CD} 8^{+} \mathrm{T}$ lymphocytes. The activation of $\mathrm{T}$ cells results in an inflammatory demyelination of the CNS (Huseby et al., 2001;Schluesener and Wekerle, 1985;Sedgwick and Mason, 1986). The activated T cells were able to cross the blood-brain-barrier (BBB) and enter the CNS (Hickey et al., 1991), where they appeared to compromise BBB integrity and target one or more myelin antigens (Westland et al., 1999). In addition to $T$ cells, B cells were reported to play a role in the pathogenesis of MS. T and B cells of MS patients, which were reactive to myelin, showed a memory or activated phenotype and were able to activate $\mathrm{CD} 4^{+} \mathrm{T}$ cells (Lovett-Racke et al., 1998;Scholz et al., 1998).

\subsubsection{Pathology of multiple sclerosis}

The hallmarks of MS pathology are focal demyelinated plaques with partial axonal preservation and reactive glial scar formation in the white and grey matter of the CNS. Furthermore diffuse damage is present throughout the NAWM and grey matter, which is associated with increasing global brain atrophy during disease progression. These characteristics are valid for all MS patients, but their relative contribution to the global pathology varies between patients and the clinical subtypes of MS (Kutzelnigg and Lassmann, 2014).

Demyelinated plaques are distributed randomly throughout the CNS with preferences for periventricular and subcortical white matter, optic nerves, the cerebellar white matter, the pons and medulla, and the spinal cord, the latter most frequently in the cervical portions (Kutzelnigg and Lassmann, 2014). Demyelination is the most important diagnostic criterion for classifying a MS lesion and was reported to be associated with axonal injury and reactive astrogliosis (Ferguson et al., 1997;Kutzelnigg and Lassmann, 2014; Trapp et al., 1998). 
Axonal injury or damage leads to irreversible axonal loss and may accumulate over time. As a consequence chronic disabilities may evolve (Bjartmar and Trapp, 2001). Especially in chronic MS lesions is axonal loss pervasive but highly variable. Axonal damage might result in axonal transection and thereby provoke Wallerian degeneration (Dziedzic et al., 2010). The distal part of the axon that is separated from its cell body degenerates anterogradely, a phenomenon which is known as Wallerian degeneration. This degenerating process was reported to be a major component of axonal pathology in MS lesions and the surrounding periplaque white matter (Dziedzic et al., 2010). Although axonal damage is associated with sites of demyelination and inflammation, it occurs also independently of demyelination and is assumed to be caused by both white and grey matter pathological changes (Kutzelnigg and Lassmann, 2014).

The activity of MS lesions can be classified by myelin degradation products in macrophages/ microglia and macrophage activation (Brück et al., 1995). The degradation of minor myelin proteins such as MOG and CNPase takes a few days, while the major myelin degradation products remain for six to ten days. Macrophages with lipid degradation products persist in lesions for several months.

In early active lesions the macrophages contained myelin degradation products that were stained with LFB and were immunoreactive with all major myelin proteins including CNPase and MOG.

Late active lesions showed more advanced myelin degradation with myelin debris stained with LFB and immunoreactive for MBP and PLP, but not MOG and CNPase. A lesion with macrophages containing empty or PAS-positive vacuoles was referred to as an inactive demyelinated lesion.

Early remyelinating plaques in which the process of remyelination is ongoing, exhibited not only an inflammatory infiltrate but also clusters of axons surrounded by a thin myelin sheath. Late remyelinated plaques (shadow plaques) revealed only few macrophages, axons with thin myelin sheaths and astrogliosis (Brück et al., 1995). Once lesions exhibits an inactive lesion center, they are described as chronic lesions (Kutzelnigg and Lassmann, 2014).

\subsection{Cuprizone mouse model}

Several animal models are available for investigation of pathological mechanisms in the CNS which partially mimic the pathology found in MS. The most common model to evaluate autoinflammatory disease mechanisms is experimental autoimmune encephalomyelitis (EAE). This autoimmunity is usually induced by injection of a peptide or protein naturally located in the CNS. The resulting autoimmunity causes demyelination, inflammation and tissue damage in the CNS, preferentially in the spinal 
cord. However, the remyelination is difficult to assess in this model since demyelination and remyelination occur concurrently and an area of incomplete demyelination cannot be easily distinguished from an area of remyelination (Gudi et al., 2014;Matsushima and Morell, 2001;Skripuletz et al., 2011). Thus, animal models of toxic demyelination such as the cuprizone mouse model are used which exhibited a synchronous and anatomically reproducible course of de- and remyelination (Matsushima and Morell, 2001;Skripuletz et al., 2011).

The neurotoxin cuprizone was established in the 1960s for different species (Carlton, 1967) and was used at high concentrations to induce scrapie-like spongiform encephalopathy in rats, mice, guinea pigs and hamsters (Carlton, 1967;Carlton, 1969). High dosages of cuprizone (e.g. 0.5\%) resulted in giant hepatic mitochondria in mice and were very toxic when administered early during development (Flatmark et al., 1980;Kesterson and Carlton, 1972;Suzuki, 1969). Mice treated with high dosage of cuprizone showed brain edema, demyelination, astrogliosis and hydrocephalus (Pattison and Jebbett, 1971a;Pattison and Jebbett, 1971b).

Mice fed with 0.2 to $0.25 \%$ cuprizone showed consistent demyelination of the corpus callosum and cortex after five to six weeks of treatment (Hiremath et al., 1998;Skripuletz et al., 2011). The extent of demyelination is dependent on the mouse strain and brain region analyzed (Hiremath et al., 1998;Jurevics et al., 2002; Lindner et al., 2008;Taylor et al., 2009;Taylor et al., 2010). The first pathological alteration that can be assessed is oligodendroglial apoptosis after two days of cuprizone administration, which reaches a maximum after 10 to 21 days of cuprizone treatment (Buschmann et al., 2012; Hesse et al., 2010). The loss of oligodendrocytes leads to subsequent microgliosis, astrogliosis, demyelination and axonal damage in the following four to five weeks of cuprizone ingestion (Hiremath et al., 1998;Lindner et al., 2009). It was demonstrated that this model is devoid of any significant adaptive immune response as $\mathrm{Rag}^{-/-}$mice lacking $T$ and $B$ cells were indistinguishable from wildtype mice (Hiremath et al., 2008; Matsushima and Morell, 2001). Moreover, the blood-brain-barrier (BBB) remained intact in the cuprizone model (Bakker and Ludwin, 1987;Kondo et al., 1987;McMahon et al., 2002).

Although the exact mechanism of cuprizone-induced oligodendroglial apoptosis is not yet understood, a recent study demonstrated that cuprizone is selectively toxic for mature oligodendrocytes and not for neurons, astrocytes or microglia in vitro (Benardais et al., 2013). It is suggested that the copper chelator cuprizone induces copper deficiency, which may cause the toxic effects. However, the administration of copper 
during cuprizone treatment did not reduce the toxic effects induced by cuprizone (Carlton, 1967).

After the cuprizone diet is discontinued, remyelination occurs within a few days and is completed approximately one to four weeks after cuprizone diet cessation, depending on the concentration of cuprizone used in the diet, the treatment period and the region analyzed (Blakemore, 1973; Lindner et al., 2009;Matsushima and Morell, 2001; Skripuletz et al., 2011).

Thus, the well characterized cuprizone model with a distinct sequence of pathological changes including demyelination and remyelination is an appropriate model to investigate axonal damage in relation to remyelination.

\section{$1.3 \quad$ Aims}

Acute axonal damage is observed during demyelination and is also detectable in remyelinating or remyelinated MS lesions. Remyelination is accompanied by ion channel redistribution and elevated mitochondria content. These factors might be able to influence the viability of axons.

Therefore, the first aim of the project was to investigate the temporal and functional relation of early remyelination and acute axonal damage. To analyze the relationship between these processes the well characterized cuprizone mouse model was used. To verify the relevance of the results for MS, remyelinating MS lesions were examined.

The potassium channel inhibitor 4-aminopyridine and the acid sensing ion channel inhibitor amiloride were shown to be beneficial in EAE. Thus, in the second part of my work, the capability of these ion channel inhibitors to preserve the integrity of axons during demyelination and remyelination was evaluated in the cuprizone mouse model. 


\section{MATERIALS AND METHODS}

\section{$2.1 \quad$ Materials}

\subsubsection{Chemicals/ reagents}

chemicals/ reagents

4-aminopyridine

Acetic acid (100\% solution)

Amiloride

ARALDITE Hardener HY 964

ARALDITE ${ }^{\circledR}$ Accelerator DY 964

Azure II, powder

Borax (di-sodium tetraborate

decahydrate)

BSA (bovine serum albumine)

Citric acid monohydrate

Cuprizone

(bis(cyclohexanone)oxaldihydrazone)

CuSO4

DAB (3,3'-diaminobenzidine)

DAPI (4',6-diamidino-2-phenylindole)

DePex mounting medium

Dako Fluorescent Mounting Medium

EDTA (ethylenediaminetetraacetic acid)

Ethanol, 96\%

FCS (fetal calf serum)

Formalin (37\% solution, free from acid)

Glutaraldehyde (25\% aqueous solution) provider

Sigma-Aldrich, Germany

Merck, Millipore, Germany

Sigma-Aldrich, Germany

Serva Electrophoresis GmbH, Germany

Serva Electrophoresis GmbH, Germany

Merck, Millipore, Germany

Merck, Millipore, Germany

Serva Electrophoresis GmbH, Germany

Merck, Millipore, Germany

Sigma-Aldrich, Germany

Sigma-Aldrich, Germany

Sigma-Aldrich, Germany

Molecular Probes, life technologies,

Germany

Serva Electrophoresis GmbH, Germany

Dako Deutschland GmbH, Germany

Merck KGaA, Germany

Merck, Millipore, Germany

Biochrom AG, Germany

Merck, Millipore, Germany

Merck KGaA, Germany 
chemicals/ reagents

Ground mouse chow (complete feed for rat \& mice maintenance, ground)

$\mathrm{H}_{2} \mathrm{O}_{2}$ (30\% solution)

$\mathrm{HCl}(25 \%, 1 \mathrm{~mol} / \mathrm{I}, 0.1 \mathrm{~mol} / \mathrm{l})$

Horse serum

Isoflurane

Isopentane

Isopropyl alcohol (99,5\% solution)

LFB (Luxol fast blue)

Lithium carbonate

Mayer's hemalum solution

Methylene blue

$\mathrm{NaOH}$

$\mathrm{NaOH}(1 \mathrm{~mol} / \mathrm{I}, 0,1 \mathrm{~mol} / \mathrm{l})$

$\mathrm{NaCl}$

Osmium tetroxide

Paraffin (Paraplast Plus)

PBS (Dulbecco's PBS, 10x powder)

Periodic acid

PFA (paraformaldehyde)

Propylenoxide

Qiazol Lysis Reagent

Random Hexamers

Renlam M-1

Sucrose

\section{provider}

Ssniff Spezialdiäten GmbH, Germany

Merck, Millipore, Germany

Merck, Millipore, Germany

Sigma-Aldrich, Germany

Abbvie, Germany

Sigma-Aldrich, Germany

Merck, Millipore, Germany

Sigma-Aldrich, Germany

Merck KGaA, Germany

Merck, Millipore, Germany

Merck, Millipore, Germany

Merck, Millipore, Germany

Merck KGaA, Germany

Merck KGaA, Germany

Carl Roth, Germany

McCormick Scientific, Leica, Germany

Applichem, Germany

Merck KGaA, Germany

Sigma-Aldrich, Germany

Serva Electrophoresis GmbH, Germany

Quiagen, Germany

Invitrogen, life technologies, Germany

Serva Electrophoresis GmbH, Germany

Sigma-Aldrich, Germany 
chemicals/ reagents

TissueTek O.C.T. Compound

Triton $\times 100$

Trizma base

Uranyl acetate

Xylol provider

Sakura, Netherlands

MP Biomedicals, Germany

Sigma-Aldrich, Germany

Merck KGaA, Germany

Merck, Millipore, Germany

\subsubsection{Enzymes/ proteins}

\section{Enzymes/ proteins}

ExtrAvidin-Peroxidase ( $0.01 \%$ solution)

Trypsin (2.5\%)

Trypsin-EDTA $(0,05 \%)$ provider

Sigma-Aldrich, Germany

Sigma-Aldrich, Germany

Gibco, life technologies, Germany

\subsubsection{Kits}

Kits

qPCR Core Kit

RNeasy Mini Kit

TaqMan MicroRNA Reverse

Transcription Kit provider

Eurogentic, Germany

Quiagen, Germany

Applied Biosystems, life technologies,

Germany

\subsubsection{Equipment/ Instruments}

\section{Equipment/ Instruments}

Camera for fluorescence microscope

XM10

Camera for light microscope DP71

Excelsior AS tissue processor

\section{Provider}

Olympus, Germany

Olympus, Germany

Thermo Scientific, USA 
Equipment/ Instruments

Fluorescence microscope BX51

Glass microscope slides (Superfrost

Plus)

iQ5 Optical System Software

iQ5 Real-Time PCR Detection System

Laser scanning confocal microscope

FluoView FV1000

Light microscope BX41

Microtome SM2000R

Microwave NN E201 WM

Motorized inverted microscope IX81

Ocular counting grid, WHSZ 10X-H

Research Cryostat CM3050

\section{Provider}

Olympus, Germany

Thermo Scientific, USA

Bio-Rad, Germany

Bio-Rad, Germany

Olympus, Germany

Olympus, Germany

Leica, Germany

Panasonic, Germany \& Austria

Olympus, Germany

Olympus, Germany

Leica, Germany

\subsubsection{Software}

Software

GraphPad Prism 5.01

FV10-ASW 4.0 viewer

FV10-ASW 4.0 (software of the Laser scanning confocal microscope FluoView FV1000)

cellSens Dimension 1.7

\section{Provider}

GraphPad, USA

Olympus, Germany

Olympus, Germany

Olympus, Germany 


\subsubsection{Tissue from MS patients analyzed}

\begin{tabular}{lccl} 
Case-number & age & sex & MS lesion \\
\hline B1149/ 06-5 & 38 & w & Early remyelinating, early active \\
B899/ 09K-2 & 41 & w & Early remyelinating, early active \\
B1024/ 09K & 41 & m & Early remyelinating, early active \\
B515/ 10K-2 & 31 & m & Early remyelinating, early active
\end{tabular}

\begin{tabular}{llll} 
Case-number & age & sex & MS lesion \\
\hline A53/ 06-9 & 50 & w & Chronic inactive \\
A2/ 10-30 & 47 & w & Chronic inactive \\
A136/ 09-2 & 45 & w & Chronic inactive (lesion 1) \\
A136/ 09-2 & 45 & w & Chronic inactive (lesion 2) \\
A50/ 90-X1B & 68 & m & Chronic inactive \\
A53/ 06-12 & 50 & w & Chronic inactive
\end{tabular}

$\begin{array}{llll}\text { A279/ 81-2 } & 44 & \text { w } & \text { Remyelinated (Shadow plaque) } \\ \text { A215/ 90-1b } & 56 & \text { m } & \text { Remyelinated (Shadow plaque) } \\ \text { A50/ 90-X1B } & 68 & \text { m } & \text { Remyelinated (Shadow plaque) } \\ \text { A53/ 06-12 } & 50 & \text { w } & \text { Remyelinated (Shadow plaque) } \\ \text { A82/ 08-1 } & 66 & \text { m } & \text { Remyelinated (Shadow plaque) }\end{array}$

\section{$2.2 \quad$ Methods}

\subsubsection{Mice}

For all in vivo experiments seven to eight week old male C57BL/6J mice were purchased by Charles River (Sulzfeld, Germany). Up to six animals were housed together on a $12 / 12 \mathrm{~h}$ light/ dark cycle and had access to food and water ad libitum. For adaptation to the new environment all in vivo experiments started one week after the arrival of the mice. 


\subsubsection{Cuprizone mouse model}

Seven to eight week old male C57BL/6J mice were fed for four to six weeks with the cupper chelator cuprizone $(0.25 \%$ in normal chow) to induce demyelination in the brain. After cuprizone withdrawal mice were fed with normal diet to analyze remyelination. At several time points during de- and remyelination brains of mice were harvested and processed for analysis by histochemistry, quantitative PCR or electron microscopy. Untreated mice were used as an appropriate control.

To investigate the role or influence of 4-aminopyridine or amiloride during de- and remyelination mice were treated either with $100 \mu \mathrm{g}$ 4-aminopyridine (twice a day, s.c.) or with $200 \mu \mathrm{g}$ amiloride (once a day, i.p.). For analysis of ion channel inhibition during remyelination, mice were treated either with the unspecific voltage-gated potassium channel inhibitor 4-aminopyridine (twice a day, 100 $\mathrm{g} / \mathrm{mouse}$ ), the acid sensing ion channel (ASIC) inhibitor amiloride (once a day, 200 $\mathrm{g} / \mathrm{mouse}$ ) or vehicle (phosphate buffered saline, PBS) after six weeks of cuprizone challenge. In another approach 4aminopyridine was administered a few days preceding cuprizone withdrawal, to achieve higher levels of the drug at the onset of remyelination. For analysis of ion channel inhibition during demyelination, mice received cuprizone diet for three weeks and the ion channel inhibitors 4-aminopyridine and amiloride were administered during the following three weeks of cuprizone challenge.

\subsubsection{Histology}

Mice were anesthetized with an overdose of $14 \%$ chloral hydrate solution in bidistilled water and transcardially perfused with PBS followed by $4 \%$ paraformaldehyde (PFA) solution in PBS. Heads were further fixed in the same buffer for another two days and then embedded in paraffin. For embedding paraffin the brains of mice were washed with PBS after fixation with 4\% PFA solution and cut into 2-3mm coronal slices. For this purpose the slices were dehydrated by a series of isopropanol/ xylol and then transferred into paraffin by an automatic embedding machine (Excelsior AS tissue processor). For histological evaluation $1 \mu \mathrm{m}$ sections $(0.5 \mathrm{~mm}$ from bregma line according to the mouse brain atlas by G. Paxinos and K.B.J. Franklin, Academic Press, 1997) were cut by a microtome and mounted onto glass slides. The sections were then deparaffinized ( $1 \mathrm{~h}$ at $60^{\circ} \mathrm{C}, 4$ times for $10 \mathrm{~min}$ in xylol) followed by rehydration (5min in: xylol/ Isopropanol, 2 times 100\% Isopropanol, 90\% Isopropanol, 70\% Isopropanol and 50\% Isopropanol). After washing the sections in distilled water histochemistry or immunohistochemistry was performed. The sections were rehydrated by using the xylol/ Isopropanol series in a reversed order and mounted with DePex mounting medium. 
For frozen sections mice were perfused as described above. Brains were collected directly after perfusion and stayed at $4{ }^{\circ} \mathrm{C}$ for $2 \mathrm{~h}$ in a $4 \%$ PFA solution. For preservation of the tissue during the freezing process the brains were incubated in $20 \%$ sucrose solution until sunk to the bottom (approximately after 24h). The tissue was embedded in TissueTek on cork slides and frozen in isopentane which was cooled down by liquid nitrogen. The embedded tissue was cut into $6-7 \mu \mathrm{m}$ sections by a cryostat. The sections were desiccated at room temperature and washed once in PBS. Afterwards all histochemical or immunohistochemical procedures were performed as usual.

\subsubsection{Luxol fast blue-periodic acid Schiff (LFB-PAS) staining}

LFB-PAS staining was used to stain myelin to determine the demyelinated areas (KLUVER and BARRERA, 1953). Sections were rehydrated to 90\% isopropanol, transferred into LFB working solution (0.1\% LFB (w/ v) and $0.5 \%$ acetic acid in Ethanol) and incubated at $60^{\circ} \mathrm{C}$ over night. The solution was changed to $90 \%$ isopropanol and the staining was differentiated until only the myelin remains in dark blue by a series of $0.05 \%$ lithium carbonate, $70 \%$ isopropanol and distilled water. The sections were incubated for 5 min in $1 \%$ periodic acid and 5 min in tap water. After washing the slides thoroughly with distilled water the sections were transferred into Schiff's reagent for 20 min and washed again 5min with tap water. The slides were incubated for 2 min in Mayers hemalum solution (1:1 in bidistilled water) to stain the nuclei of cells, washed in distilled water, differentiated with $\mathrm{HCl}$-Isopropanol $(70 \%$ Isopropyl alcohol, $0.25 \% \mathrm{HCl})$, and washed for 10 min with tap water.

\subsubsection{Immunohistochemistry and fluorescence immunohistochemistry}

Primary antibodies utilized were against APP (amyloid precursor protein; acute axonal damage), Mac3 (activated microglia), GFAP (glial fibrilliary acidic protein; activated astrocytes), Olig2 (oligodendrocyte transcription factor 2; mature oligodendrocytes and oligodendrocyte progenitors), NogoA (neurite outgrowth inhibitor A, mature oligodendrocytes) and p25 (mature oligodendrocytes). Biotinylated secondary antibodies, peroxidase conjugated avidin and DAB were used for immunohistochemistry. Fluorescence labeled secondary antibodies were used for fluorescence immunohistochemistry.

The tissue was fixed with PFA to preserve the morphology which leads to a masking of some antibody epitopes. Thus, for some immunhistochemical stainings the retrieval of epitopes is needed. For the retrieval of epitopes the sections were heated in a microwave either in $10 \mathrm{mM}$ citrate buffer $(\mathrm{pH} 6.0)$ or in Tris-EDTA buffer (10mM Tris, 1mM EDTA, pH 9.0) 5 times for $3 \mathrm{~min}, 3$ times for 5-7 min or 2 times for $10 \mathrm{~min}$. After 
each heating cycle evaporated solution was replaced either by buffer or bidistilled water. After this procedure the sections had to cool down at room temperature for approximately $30 \mathrm{~min}$ and were then washed with distilled water. Endogenous peroxidase was blocked by incubating the slides in $3 \% \mathrm{H}_{2} \mathrm{O}_{2}$ in PBS for 20 min at $4^{\circ} \mathrm{C}$ and subsequently washing the slides with PBS. To minimize unspecific antibody binding the sections were incubated in blocking buffer (see Table 1) for 20 min in a wet chamber. The blocking buffer was also used for dilution and incubation with primary and secondary antibodies (see Table 1 and Table 2) as well as for the incubation with peroxidase conjugated avidin (ExtrAvidinPeroxidase). The sections were incubated with primary antibodies over night at $4^{\circ} \mathrm{C}$ and washed afterwards three times with PBS. The sections were then incubated with the secondary antibodies for $1 \mathrm{~h}$ at room temperature and washed three times with PBS. For fluorescence double immunohistochemistry, the sections were incubated consecutively with the primary and secondary antibodies and washed with PBS three times between the incubations. DAPI $(1: 10,000)$ was applied onto the slides to stain the nuclei for $10-15 \mathrm{~min}$ at room temperature. The slides were then washed three times with PBS and once in bidistilled water and were mounted with Dako Fluorescence Mounting Medium.

Table 1: List of primary antibodies for immunhistochemistry and fluorescence immunohistochemistry

\begin{tabular}{|l|l|l|l|l|l|l|}
\hline Antigen & Identification of & Host & Dilution & $\begin{array}{l}\text { Antigen } \\
\text { retrieval }\end{array}$ & $\begin{array}{l}\text { Blocking } \\
\text { buffer }\end{array}$ & Provider \\
\hline APP & $\begin{array}{l}\text { acute axonal } \\
\text { damage }\end{array}$ & $\begin{array}{l}\mathrm{Ms} / \\
\mathrm{mc}\end{array}$ & $1: 2,000$ & Citrat & $\begin{array}{l}10 \% \mathrm{FCS} / \\
\mathrm{PBS}\end{array}$ & $\begin{array}{l}\text { Chemicon/ } \\
\text { MAB348 }\end{array}$ \\
\hline Caspr & paranode & $\begin{array}{l}\mathrm{Rbb} / \\
\mathrm{pc}\end{array}$ & $1: 1,000$ & & $\begin{array}{l}10 \% \mathrm{HS} / \\
1 \% \mathrm{BSA} / \\
\mathrm{PBS}\end{array}$ & $\begin{array}{l}\text { abcam/ } \\
\text { ab34151 }\end{array}$ \\
\hline $\begin{array}{l}\text { CNP- } \\
\text { ase }\end{array}$ & myelin protein & $\begin{array}{l}\mathrm{Ms} / \\
\mathrm{mc}\end{array}$ & $1: 200$ & Citrat & $\begin{array}{l}10 \% \mathrm{FCS} / \\
\mathrm{PBS}\end{array}$ & $\begin{array}{l}\text { Covance/ } \\
\text { SMI-91R }\end{array}$ \\
\hline GFAP & activated astrocyte & $\begin{array}{l}\mathrm{Ms} / \\
\mathrm{mc}\end{array}$ & $1: 1,000$ & $\begin{array}{l}10 \% \mathrm{FCS} / \\
\mathrm{PBS}\end{array}$ & $\begin{array}{l}\text { DAKO/ REF } \\
\text { Z0334 }\end{array}$ \\
\hline $\begin{array}{l}\text { Mac3 } \\
\text { [M3/84] }\end{array}$ & activated microglia & $\begin{array}{l}\mathrm{Rat} / \\
\mathrm{pc}\end{array}$ & $1: 200$ & Citrat & $\begin{array}{l}10 \% \mathrm{FCS} / \\
\mathrm{PBS}\end{array}$ & $\begin{array}{l}\text { BD } \\
\text { Pharmingen/ } \\
553322\end{array}$ \\
\hline MBP & myelin protein & $\begin{array}{l}\mathrm{Rb} / \\
\mathrm{pc}\end{array}$ & $1: 2,000$ & & $\begin{array}{l}10 \% \mathrm{FCS} / \\
\mathrm{PBS}\end{array}$ & $\begin{array}{l}\text { DAKO/ REF } \\
\text { A0623 }\end{array}$ \\
\hline
\end{tabular}


Table 1 continued

\begin{tabular}{|c|c|c|c|c|c|c|}
\hline Antigen & Identification of & Host & Dilution & $\begin{array}{l}\text { Antigen } \\
\text { retrieval }\end{array}$ & $\begin{array}{l}\text { Blocking } \\
\text { buffer }\end{array}$ & Provider \\
\hline MOG & myelin protein & $\begin{array}{l}\text { Rat/ } \\
\text { pc }\end{array}$ & $1: 1,000$ & Citrat & $\begin{array}{l}10 \% \mathrm{FCS} / \\
\text { PBS }\end{array}$ & $\begin{array}{l}\text { Gift from D. } \\
\text { Merkler }\end{array}$ \\
\hline $\begin{array}{l}\text { NogoA } \\
11 C 7\end{array}$ & $\begin{array}{l}\text { mature } \\
\text { oligodendrocyte }\end{array}$ & $\begin{array}{l}\mathrm{Ms} / \\
\mathrm{mc}\end{array}$ & $1: 5,000$ & Citrat & $\begin{array}{l}10 \% \mathrm{FCS} / \\
\text { PBS }\end{array}$ & $\begin{array}{l}\text { Gift from } \\
\text { M.Schwab, } \\
\text { Zürich } \\
\text { (Oertle et al. } \\
\text { 2003) }\end{array}$ \\
\hline Olig2 & $\begin{array}{l}\text { OPCs and mature } \\
\text { oligodendrocyte }\end{array}$ & $\begin{array}{l}\mathrm{Rbb} / \\
\mathrm{pc}\end{array}$ & $1: 300$ & $\begin{array}{l}\text { Tris- } \\
\text { EDTA }\end{array}$ & $\begin{array}{l}10 \% \mathrm{FCS} / \\
\text { PBS }\end{array}$ & IBL/ 18953 \\
\hline $\begin{array}{l}\text { P25 } \\
\text { [EPR33 } \\
16]\end{array}$ & $\begin{array}{l}\text { mature } \\
\text { oligodendrocyte }\end{array}$ & $\begin{array}{l}\mathrm{Rbb} / \\
\mathrm{pc}\end{array}$ & $1: 100$ & $\begin{array}{l}\text { Tris- } \\
\text { EDTA }\end{array}$ & $\begin{array}{l}10 \% \\
\text { NGS/ } \\
\text { PBS }\end{array}$ & $\begin{array}{l}\text { abcam/ } \\
\text { ab92305 }\end{array}$ \\
\hline $\begin{array}{l}\text { PLP } \\
\text { clone } \\
\text { plpc1 }\end{array}$ & myelin & $\begin{array}{l}\mathrm{Ms} / \\
\mathrm{mc}\end{array}$ & $1: 500$ & Citrat & $\begin{array}{l}10 \% \mathrm{FCS} / \\
\text { PBS }\end{array}$ & $\begin{array}{l}\text { ABD } \\
\text { Serotec/ } \\
\text { MCA 839G }\end{array}$ \\
\hline
\end{tabular}

For conventional immunohistochemistry, slides were incubated with peroxidase conjugated avidin for $1 \mathrm{~h}$ at room temperature, washed three times with PBS and incubated in a DAB working solution (0.05\% DAB (w/ v) and $0.012 \% \mathrm{H}_{2} \mathrm{O}_{2}$ ) for 1-30min. Oxidization of $\mathrm{DAB}$ by peroxidase lead to a brown or dark brown labeling at sites of antibody binding. The conventional immunohistochemistry on human sections was amplified by incubation of the slides for $15-20$ min in $\mathrm{CuSO}_{4}$ working solution $\left(2 \% \mathrm{CuSO}_{4}\right.$, $0.8 \% \mathrm{NaCl})$.

Table 2: List of secondary antibodies for immunhistochemistry and fluorescence immunohistochemistry

\begin{tabular}{|l|l|l|l|l|}
\hline Antibody & Modification & Host & Dilution & Provider \\
\hline anti-mouse IgG & biotinylated & sheep & $1: 200$ & GE Healthcare/ RPN1001 \\
\hline anti-mouse IgG & $\begin{array}{l}\text { Cy3 } \\
\text { conjugated }\end{array}$ & goat & $1: 200$ & $\begin{array}{l}\text { Jackson ImmunoResearch/ } \\
115-165-146\end{array}$ \\
\hline
\end{tabular}


Table 2 continued

\begin{tabular}{|l|l|l|l|l|}
\hline Antibody & Modification & Host & Dilution & Provider \\
\hline anti-rabbit IgG & biotinylated & goat & $1: 200$ & $\begin{array}{l}\text { Jackson ImmunoResearch/ } \\
111-065-144\end{array}$ \\
\hline anti-rabbit IgG & $\begin{array}{l}\text { Alexa 488 } \\
\text { conjugated }\end{array}$ & goat & $1: 200$ & $\begin{array}{l}\text { Molecular Probes } \AA \text {, life } \\
\text { technologies/ A11034 }\end{array}$ \\
\hline anti-rat IgG & biotinylated & goat & $1: 200$ & GE Healthcare/ RPN1005 \\
\hline anti-rat IgG & biotinylated & goat & $1: 1,000$ & $\begin{array}{l}\text { DCS Innovative diagnostic } \\
\text { system/ REF RS000C01 }\end{array}$ \\
\hline
\end{tabular}

\subsubsection{Electron microscopy}

Remaining myelin sheaths and new myelin sheaths cannot be distinguished by staining of myelin (LFB-PAS) or immunohistochemistry for myelin proteins (e.g. MBP, MOG) in mice. Therefore the brains of mice were analyzed by electron microscopy. Mice were anaesthetized with a lethal dose of $14 \%$ chloral hydrate, were perfused with PBS and subsequently by perfusion with $100 \mathrm{ml} \mathrm{KS}$-fixative (4\% PFA and $2.5 \%$ Glutaraldehyde in PBS) per mouse for approximately $1 \mathrm{~h}$. The brains were collected immediately after perfusion and were incubated overnight at $4^{\circ} \mathrm{C}$ in $\mathrm{KS}$-fixative. The brains were cut into 2$3 \mathrm{~mm}$ slices and a distinct region from the corpora callosa $(0.5 \mathrm{~mm}$ from bregma line according to the mouse brain atlas by G. Paxinos and K.B.J. Franklin, Academic Press, 1997) was collected and transferred into 3\% Glutaraldehyde solution in PBS and were incubated in this solution at $4^{\circ} \mathrm{C}$ for at least seven days post-fixation. From these pieces of the corpus callosum $1 \mathrm{~mm}$ thick para-sagittal slices were cut, processed through osmium tetraoxide, dehydrated and embedded in synthetic resin. To determine alterations in demyelination and tissue damage of the corpus callosum semi- and ultrathin sections $(300 \mathrm{~nm})$ were generated. The semithin section were stained with Richardson's stain (RICHARDSON et al., 1960) and evaluated by light microscopy while the ultrathin sections were analyzed by electron microscopy.

\subsubsection{Morphometry and data aquisition}

The level of demyelination of the corpus callosum after cuprizone treatment was assessed in LFB-PAS stained sections by a semi quantitative score: no demyelination (0), 0-33\% demyelination (1), 33\%-66\% demyelination (2) and more than 66\% demyelination (3). $\mathrm{APP}^{+}$spheroids and the number of glial cells $\left(\mathrm{Mac}^{+}, \mathrm{GFAP}^{+}, \mathrm{Olig}^{+}\right.$or $\mathrm{NogoA}^{+}$cells) were determined for the corpus callosum by counting spheroids and cells 
at $400 x$ with an ocular counting grid. To evaluate acute axonal damage in the context of early remyelination, fluorescence double immunohistochemistry (APP with MBP, APP with Caspr or APP with GFAP) was analyzed by confocal microscopy. Therefore 20-30 randomly selected visual fields which had at least one $\mathrm{APP}^{+}$spheroid were measured per corpus callosum at $200 x$ with $9 \%$ zoom or $400 x$ with $7 \%$ zoom. The degree of association -no, partially or complete- of APP with MBP, APP with Caspr or APP with GFAP was assessed by a qualitative evaluation using the software FV10-ASW 4.0 viewer. The data were calculated as per cent of all measured APP ${ }^{+}$spheroids per corpus callosum.

Autopsy and biopsy tissue specimens of brains of MS patients, which exhibited early remyelinating early active, chronic inactive or remyelinated lesions, were analyzed. The different types of MS lesions were determined by Dr. Andreas Junker and Prof. Dr. Christine Stadelmann-Nessler according to criteria described elsewhere (Brück et al., 1994;Brück et al., 1995;Kutzelnigg et al., 2005;Lassmann, 2011;Lucchinetti et al., 2000; Schuh et al., 2014).

The acute axonal damage in the context of early remyelination was evaluated in early remyelinating early active MS lesions by fluorescence double immunohistochemistry (APP with MBP) and confocal microscopy. For qualitative analysis, 10 randomly selected visual fields were evaluated.

To assess the axon protection in remyelinated MS lesions (shadow plaques) the acute axonal damage was determined in shadow plaques and chronic inactive MS lesions by counting spheroids at $400 x$ with an ocular counting grid.

\subsubsection{Quantification of cytokines}

The level of cytokine expression in the corpora callosa was determined by quantitative PCR. Therefore mice were perfused with PBS, the brains removed and immediately cut into 2-3 $\mathrm{mm}$ coronal slices. The brain slices used to analyze cytokine expression were taken accordingly to the brain slices used for histological analysis. The brain slices were shortly freezed in an aluminum chamber on liquid nitrogen and corpora callosa were immediately excised and used for RNA isolation.

\subsubsection{Isolation of RNA}

Isolation of RNA was performed with Qiazol Lysis Reagent and RNeasy Mini Kit according to the manufacturer's instructions.

\subsubsection{Generation of CDNA}

Isolated RNA was transcribed to cDNA with TaqMan MicroRNA Reverse Transcription Kit and Random Hexamers. The reactions were performed according to the 
manufacturer's instructions with a few modifications. Briefly, one reaction consisted of 300ng of isolated RNA, 1xRT buffer, 4mM dNTPs, 10 $\mu \mathrm{M}$ Random Hexamers, $1 \mathrm{U} / \mu \mathrm{l}$ RNase Inhibitor and 2.5U/ $\mu$ l MultiScribe Reverse Transcriptase. The reverse transcription reactions run on Eppendorf Thermocycler as listed below.

$\begin{array}{lll}25^{\circ} \mathrm{C} & 10 \mathrm{~min} & \text { annealing } \\ 37^{\circ} \mathrm{C} & 120 \mathrm{~min} & \text { reverse transcription } \\ 85^{\circ} \mathrm{C} & 5 \mathrm{seC} & \text { inactivation of reverse transcriptase }\end{array}$

\subsubsection{Quantitative PCR}

The cDNA from the RT reaction was diluted to a concentration of $3 n g / \mu l$ to apply 12ng of cDNA in the qPCR reaction which was performed with qPCR Core Kit and TaqMan Expression Assays according to the manufacturers' instructions. The qPCR run according to the following protocol:

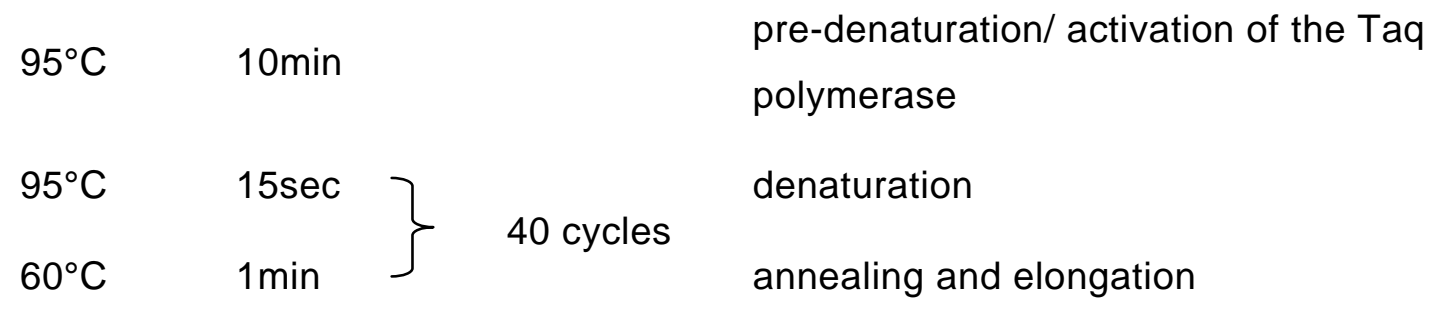

Table 3: $\quad$ List of TaqMan Expression Assays

\begin{tabular}{|l|l|l|}
\hline Gene symbol & \multicolumn{1}{|c|}{ Gene name } & \multicolumn{1}{c|}{ Provider } \\
\hline GapDH & $\begin{array}{l}\text { glyceraldehyde-3-phosphate } \\
\text { dehydrogenase }\end{array}$ & $\begin{array}{l}\text { Applied Biosystems, life } \\
\text { technologies/Mm99999915_g1 }\end{array}$ \\
\hline IL-6 & interleukin 6 & $\begin{array}{l}\text { Applied Biosystems, life } \\
\text { technologies/Mm00446190_m1 }\end{array}$ \\
\hline TGF $\boldsymbol{\beta}$ & transforming growth factor, beta 1 & $\begin{array}{l}\text { Applied Biosystems, life } \\
\text { technologies/ Mm01178820_m1 }\end{array}$ \\
\hline TNFa & tumor necrosis factor alpha & $\begin{array}{l}\text { Applied Biosystems, life } \\
\text { technologies/Mm00443258_m1 }\end{array}$ \\
\hline
\end{tabular}

\subsubsection{Statistical analysis}

Statistics were calculated using the GraphPad Prism5.01 software. The Kolmogorov-Smirnov test was carried out to test the data for normal distribution. To compare more than two groups, data were analyzed by one-way ANOVA for parametric values (Bonferroni's Multiple Comparison test). For analysis of non parametric values the 
Kruskal-Wallis test was used (Dunn's Multiple Comparison test). For comparison of two groups the unpaired t test was used for parametric values and the Mann-Whitney test was used for non parametric values. Data are displayed as mean, mean \pm SEM or as median and a P-value of $<0.05$ was considered as statistically significant. 
3.1 Investigation of acute axonal damage during early remyelination in the cuprizone mouse model and in MS lesions

\subsubsection{Efficient regeneration of the corpus callosum after cuprizone diet cessation}

To investigate axonal damage during demyelination and remyelination without significant contribution of the peripheral immune system the cuprizone mouse model was used. Mice were analyzed after six weeks of cuprizone-induced demyelination and after three weeks of recovery after cuprizone withdrawal. To examine the biological processes during early remyelination the brains of mice were isolated two to seven days after cuprizone withdrawal. For analysis of these processes at later time points of remyelination the brains of mice were isolated after two and three weeks of remyelination (Fig. 1A).
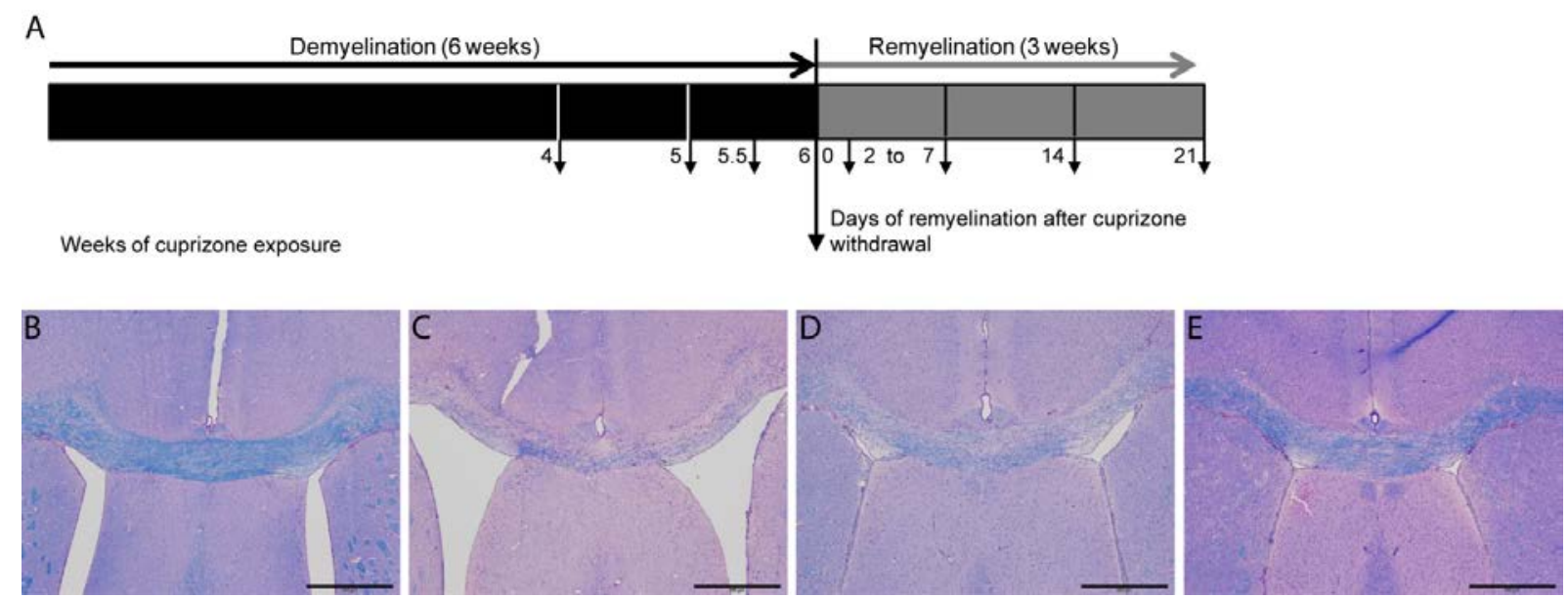

Figure 1: Design of the cuprizone experiment to investigate the acute axonal damage during early remyelination.

Seven to eight week old male C57BL/6J mice were fed for six weeks with a $0.25 \%$ cuprizone diet to induce demyelination in the brain. After cuprizone withdrawal the brain started to remyelinate. To study early remyelination brains were isolated from day two to seven after cuprizone withdrawal. Brains were isolated after two and three weeks of remyelination to study later time points of remyelination (A). Myelin was stained with LFBPAS. The majority of the corpus callosum was demyelinated after cuprizone treatment (C) compared to untreated mice (B). The medial part of the corpus callosum was preferentially remyelinated after one week of recovery (D). The corpus callosum was almost completely remyelinated three weeks after cuprizone withdrawal (E). (magnification: 20x; scale bar $=1 \mathrm{~mm}$ ) 
After six weeks of cuprizone treatment the corpus callosum was fully demyelinated. Remyelination started early after cuprizone diet cessation and was almost completed after three weeks (Fig. 1B-E).
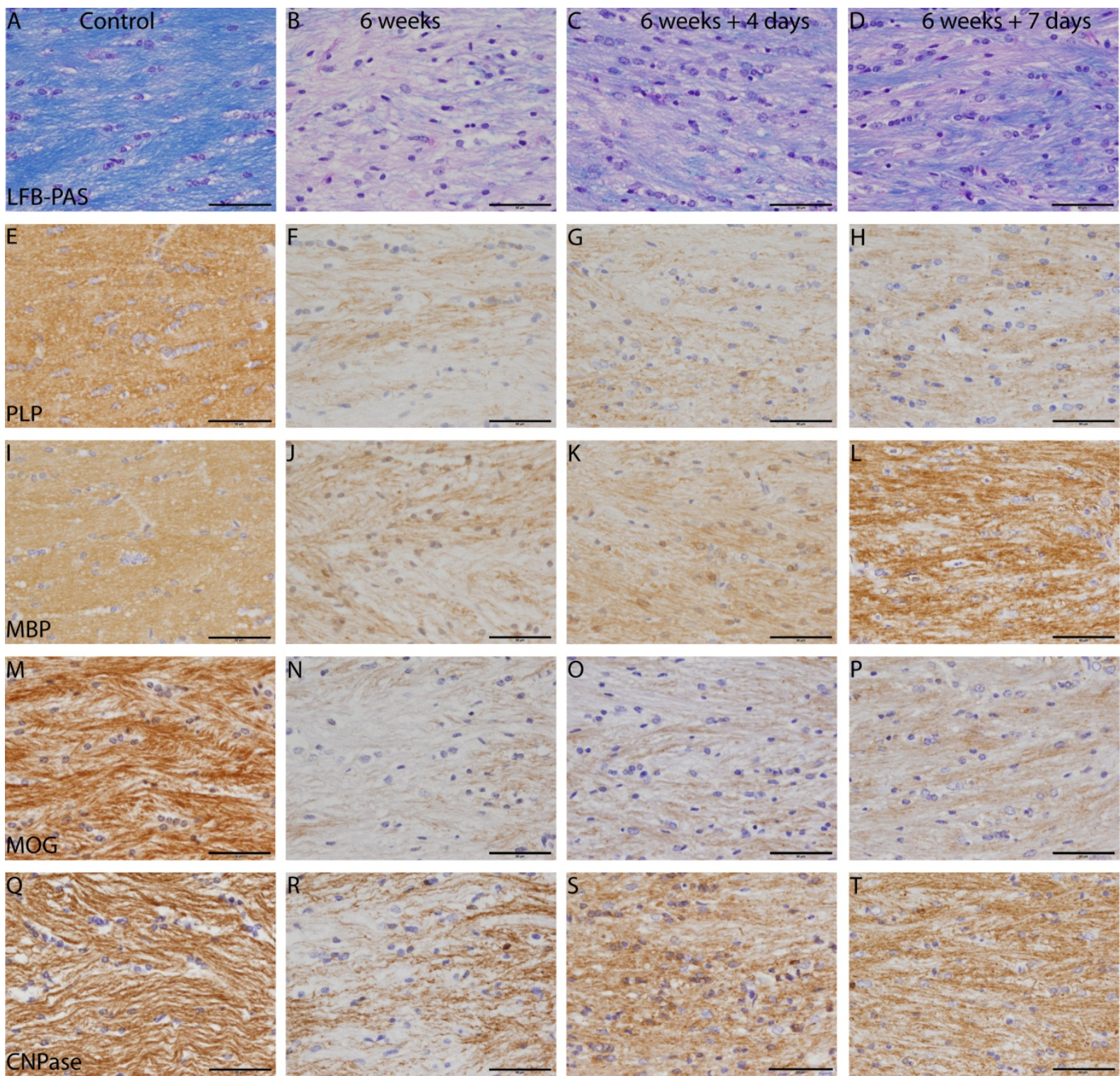

Figure 2: Remyelination as a regenerative process started within a few days after cuprizone withdrawal.

Representative images of medial corpus callosum of control mice (A, E, I, M, Q), mice after cuprizone challenge for six weeks $(\mathbf{B}, \mathbf{F}, \mathbf{J}, \mathbf{N}, \mathbf{R})$ and mice four $(\mathbf{C}, \mathbf{G}, \mathbf{K}, \mathbf{O}, \mathbf{S})$ or seven (D, H, L, P, T) days after cuprizone withdrawal. The LFB-PAS staining (A, B, C, D) indicated an almost complete demyelination of the corpus callosum after six weeks of cuprizone treatment. The remyelination was apparent at day four of remyelination. These results were also obtained by immunohistochemistry for the myelin proteins: PLP (E, $F$, $\mathbf{G}, \mathbf{H}), \operatorname{MBP}(\mathbf{I}, \mathbf{J}, \mathbf{K}, \mathbf{L}), \operatorname{MOG}(\mathbf{M}, \mathbf{N}, \mathbf{O}, \mathbf{P})$ and CNPase $(\mathbf{Q}, \mathbf{R}, \mathbf{S}, \mathbf{T})$. The immunoreactivity with the myelin proteins revealed a sequential myelin protein expression during remyelination: 1. CNP, 2. MBP, 3. MOG, 4. PLP. (magnification: 400x; scale bar $=50 \mu \mathrm{m})$ 
To determine the time course of remyelination the content of myelin was analyzed by histochemistry (LFB-PAS) and immunohistochemistry (PLP, MBP, MOG, CNPase). The evaluation of myelin content by LFB-PAS and myelin protein expression by immunohistochemistry revealed an almost complete demyelination of the corpus callosum after six weeks of cuprizone treatment (Fig. 1C; Fig. 2B, F, J, N, R). Remyelination was apparent after four days of remyelination (Fig. 2C, G, K, O, S). After one week of remyelination a major proportion of the corpus callosum was remyelinated (Fig. 2D, H, L, P, T). A sequential myelin protein expression was observed with the following sequence of myelin protein expression: 1) CNPase, 2) MBP, 3) MOG, and 4) PLP. The immunoreactivity for PLP was comparable to the LFB staining. The semi quantitative analysis of myelin content revealed a rapid decrease of the demyelinated area during the first week of recovery. The remyelination was decelerated afterwards, but was almost complete after three weeks of recovery (Fig. 3).

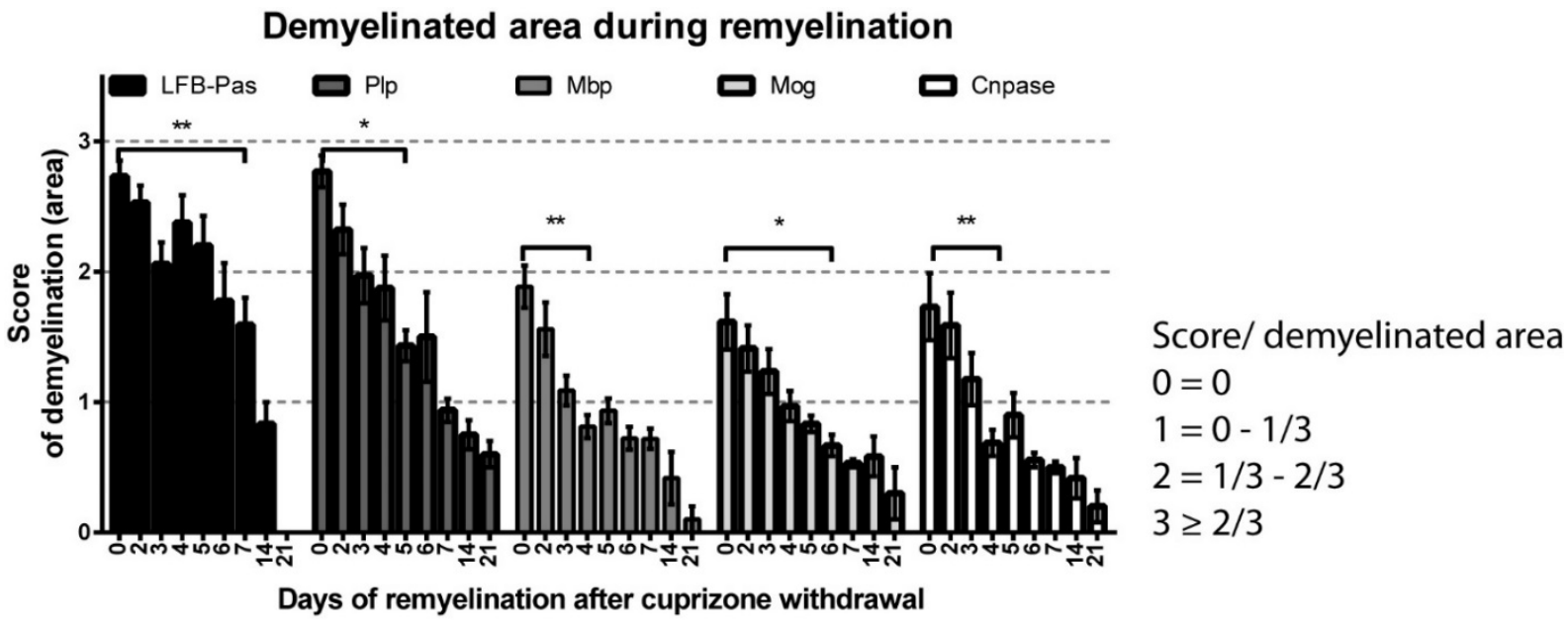

Figure 3: Semi quantitative analysis of myelin revealed rapid remyelination but sequential myelin protein expression of the corpus callosum, which started with CNP, followed by MBP, MOG, and PLP.

The demyelinated area in corpus callosum of mice was reduced during remyelination as judged by semi quantitative evaluation of LFB-PAS staining and myelin protein immunohistochemistry (PLP, MBP, MOG, CNPase). The demyelinated area was significantly reduced after four to seven days (mean \pm SEM; $n=5-17$; Kruskal Wallis test, Dunn's multiple comparison test; ${ }^{\star} p<0.05$; ${ }^{*} p<0.01$ ).

To assess the regenerative status of the brain after cuprizone challenge more precisely, oligodendrocyte precursor cells (OPCs) and mature oligodendrocytes were investigated by immunohistochemistry for Olig2 and NogoA, respectively. Although the mice had been treated continuously for six weeks with cuprizone, numerous oligodendrocyte precursor cells (Olig2 ${ }^{+}$, high expression) were present (Fig. 4B). However, only a few mature oligodendrocytes $\left(\operatorname{NogoA}^{+}\right)$were identified after cuprizone 
treatment (Fig. 4F). The corpus callosum of cuprizone-treated mice exhibited a considerable increase of Olig2 ${ }^{+}$and $\mathrm{NogoA}^{+}$oligodendrocytes four days after cuprizone withdrawal (Fig. 4C, G). The number of oligodendrocytes and their labeling intensity of Olig2 and NogoA were comparable to control mice after one week of recovery (Fig. 4A, $D, E, H)$.
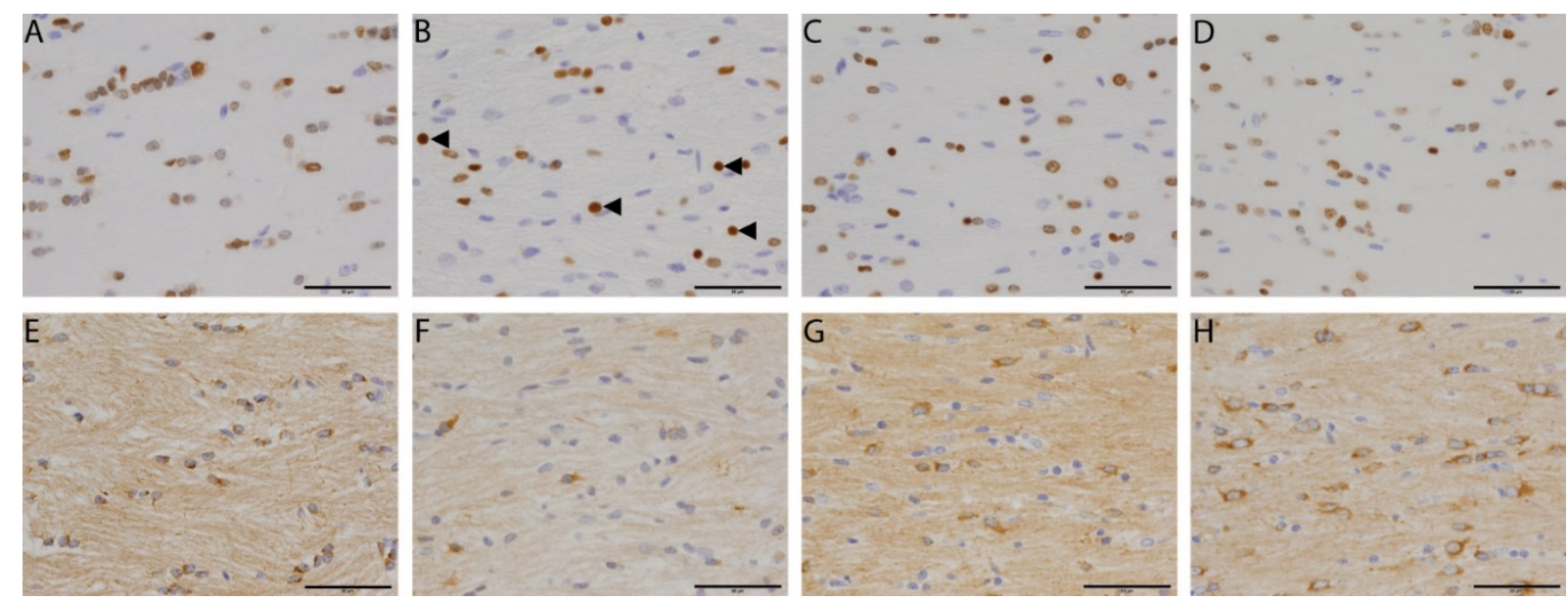

Figure 4: Numerous oligodendrocyte precursor cells were observed after six weeks of cuprizone-induced demyelination.

The number of Olig2 ${ }^{+}$oligodendrocytes was diminished in cuprizone-treated mice (B) compared to untreated mice (A). Numerous oligodendrocyte precursor cells, which expressed Olig2 at high levels (arrowheads), were observed after six weeks at the end of the cuprizone challenge (B). A few mature $\mathrm{NogoA}^{+}$oligodendrocytes were apparent after cuprizone challenge $(F)$ compared to control $(E)$. After four days of recovery increased numbers of $\mathrm{Olig2}^{+}(\mathbf{C})$ and $\operatorname{NogoA}^{+}(\mathbf{G})$ oligodendrocytes were detected. The expression of Olig2 was downregulated by oligodendrocytes seven days after cuprizone withdrawal as judged by decreased labeling intensity, indicating their differentiation (D). The differentiation of oligodendrocytes seemed to be almost completed after one week of remyelination $\mathbf{( H )}$. Representative images of medial corpus callosum were taken at $400 \mathrm{x}$ original magnification (scale bar $=50 \mu \mathrm{m})$.

The finding of increasing numbers of $\mathrm{Olig}^{+}$and $\mathrm{NogoA}^{+}$oligodendrocytes supported the impression of a rapid regenerative process during the first week after cuprizone withdrawal. The repopulation of the corpus callosum with oligodendrocytes as well as oligodendrocyte differentiation seemed to be accomplished during the first week of remyelination (Fig. 5A, B). 
A Mature Oligodendrocytes and OPCs during remyelination

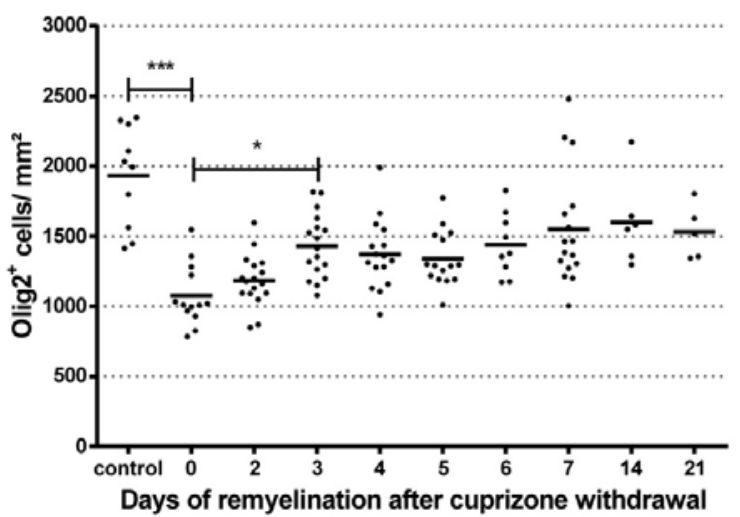

B Mature Oligodendrocytes during remyelination

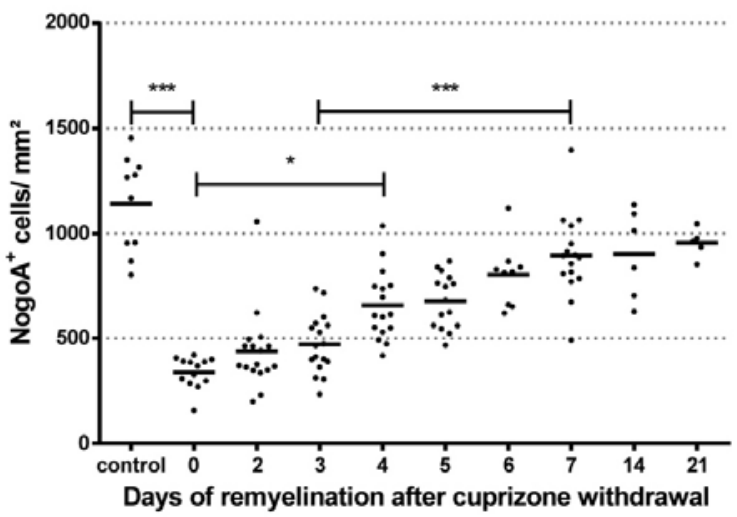

Figure 5: The number of OPCs and mature oligodendrocytes seemed to be reestablished after one week of recovery.

The number of Olig2 ${ }^{+}$oligodendrocytes was significantly decreased after cuprizoneinduced demyelination and increased significantly within three days of remyelination. After the first three days of recovery the number of Olig $2^{+}$cells remained unchanged $(\mathbf{A})$ (mean; $\mathrm{n}=5-17$; Kruskal Wallis test, Dunn's multiple comparison test; ${ }^{*} \mathrm{p}<0.05$; ${ }^{* \star *} p<0.001$ ). Corpus callosum of mice treated with cuprizone for six weeks exhibited diminished numbers of mature $\mathrm{NogoA}^{+}$oligodendrocytes. The number of $\mathrm{NogoA}^{+}$ oligodendrocytes increased during the first week of remyelination. After one week of remyelination the number of mature oligodendrocytes remained relatively stable (B) (mean; $\mathrm{n}=5-17 ;$ Kruskal Wallis test, Dunn's multiple comparison test; ${ }^{*} \mathrm{p}<0.05$; $\star * * p<0.001)$.

\subsubsection{Acute axonal damage was detectable during early and late remyelination}

To investigate the relationship between early and late remyelination and acute axonal damage, Immunohistochemistry for APP, a marker for acute axonal damage, was performed. APP is undetectable in healthy axons, but accumulates in injured axons with disturbed anterograde axonal transport. Accumulation of APP in axons can be observed a few hours to four weeks after the damaging insult (Bramlett et al., 1997;McKenzie et al., 1996;Otsuka et al., 1991;Pierce et al., 1996).

APP immunohistochemistry revealed axonal spheroids after six weeks of cuprizone treatment (309.1 $\mathrm{APP}^{+}$spheroids/ $\mathrm{mm}^{2}$ ) and during early (229.5 $\mathrm{APP}^{+}$ spheroids $/ \mathrm{mm}^{2}$ ) and late (129.2 $\mathrm{APP}^{+}$spheroids $/ \mathrm{mm}^{2}$ ) stages of remyelination (Fig. 6A$\mathrm{G})$. The number of $\mathrm{APP}^{+}$spheroids decreased gradually during remyelination, and $\mathrm{APP}^{+}$ spheroids were still present after three weeks of recovery (129.5 APP ${ }^{+}$spheroids $/ \mathrm{mm}^{2}$ ). $\mathrm{APP}^{+}$spheroids decreased significantly after six days $\left(153.0 \mathrm{APP}^{+}\right.$spheroids/ $\mathrm{mm}^{2}$ ) of remyelination compared to six weeks of demyelination (309.1 $\mathrm{APP}^{+}$spheroids/ $\mathrm{mm}^{2}$ ). Furthermore, a high variability in the density of $\mathrm{APP}^{+}$spheroids was observed between the animals (Fig. 6G). 

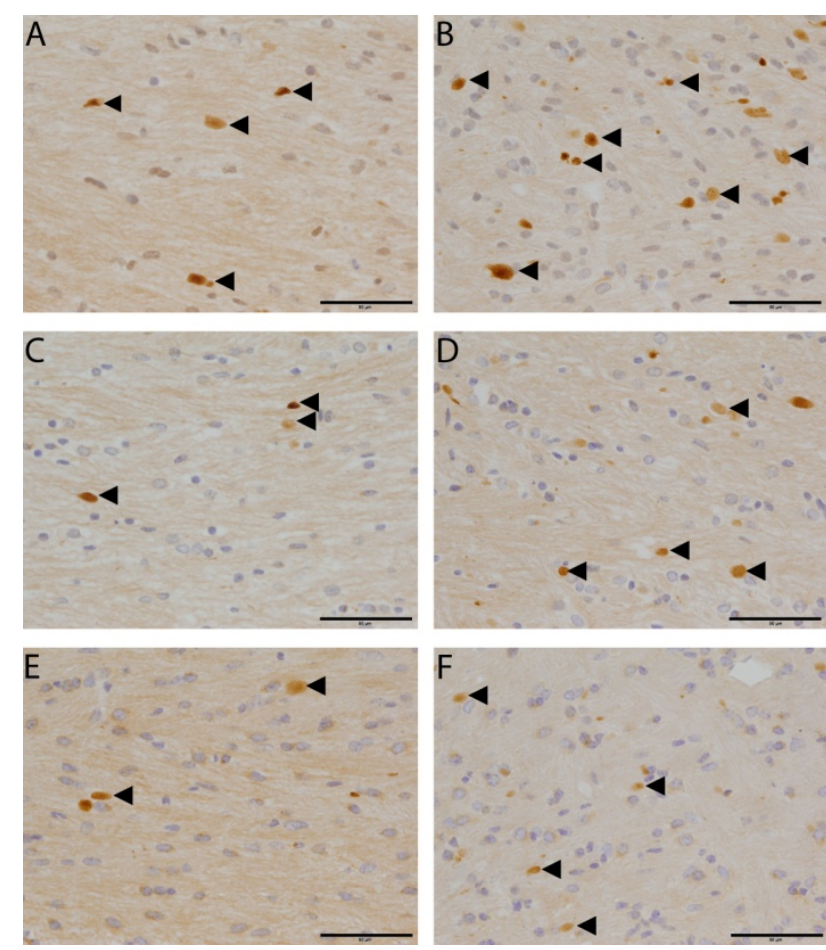

$G \quad$ Acute axonal damage during remyelination

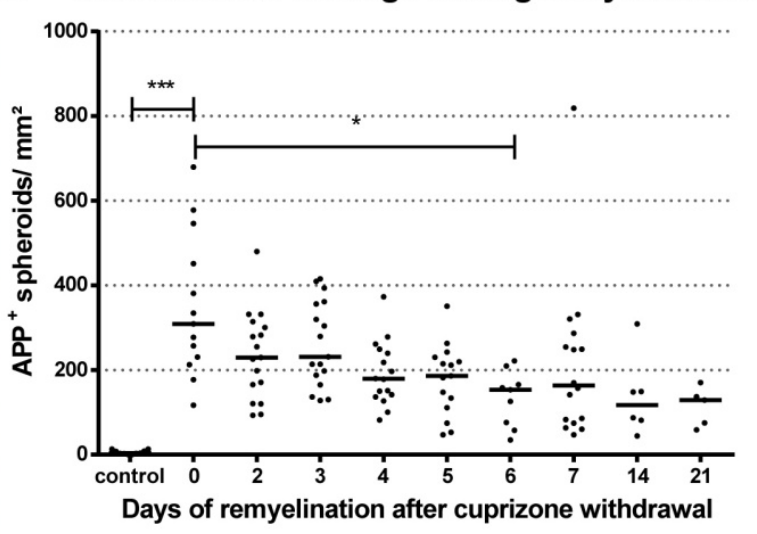

Figure 6: Acute axonal damage was detected during early and late remyelination.

Acute axonal damage (APP ${ }^{+}$spheroids) was observed after cuprizone-induced demyelination in the medial (A) and lateral (B) corpus callosum and was decreased after two days of recovery (C, D). $\mathrm{APP}^{+}$spheroids were still seen after seven days of remyelination (E, F). The density of $\mathrm{APP}^{+}$spheroids decreased continuously during remyelination (G) (median; $\mathrm{n}=5-17$; Kruskal Wallis test, Dunn's multiple comparison test; $\left.{ }^{*} p<0.05 ;{ }^{* *} p<0.001\right)$. Representative images of medial $(\mathbf{A}, \mathbf{C}, \mathbf{E})$ and lateral $(\mathbf{B}, \mathbf{D}, \mathbf{F})$ corpus callosum of cuprizone-treated mice (A, B) and of mice after two (C, D), and seven $(E, F)$ days of remyelination were taken at $400 x$ original magnification (scale bar $=50 \mu \mathrm{m}$ ).

\subsubsection{Microgliosis was declining during remyelination while astrogliosis sustained over time}

Microgliosis and astrogliosis were determined after cuprizone-induced demyelination and during remyelination to elucidate their relation to acute axonal damage by immunhistochemical staining for Mac3 and GFAP, respectively. Many activated microglia $\left(975.9 \pm 56.5\right.$ cells $\left./ \mathrm{mm}^{2}\right)$ as well as reactive astrocytes $(629.3 \pm 27.4$ cells/ $\mathrm{mm}^{2}$ ) were present after six weeks of cuprizone treatment (Fig. 7A, E, I, J). The number of $\mathrm{Mac3}^{+}$cells was significantly diminished after two days of remyelination (Fig. 7F, J) and further declined during remyelination (Fig. 7G, H, J). Mac ${ }^{+}$cells were almost absent after three weeks of recovery (26.76 \pm 9.9 cells/ $\left.\mathrm{mm}^{2}\right)$ (Fig. 7J). The immunohistochemistry for GFAP displayed the opposite situation with many reactive astrocytes (478.0 - 610.3 cells/ $\mathrm{mm}^{2}$ ) during remyelination, which was similar to six weeks of cuprizone treatment $\left(629.3 \pm 27.4\right.$ reactive astrocytes $\left./ \mathrm{mm}^{2}\right)$ (Fig. 7A-D, I). 

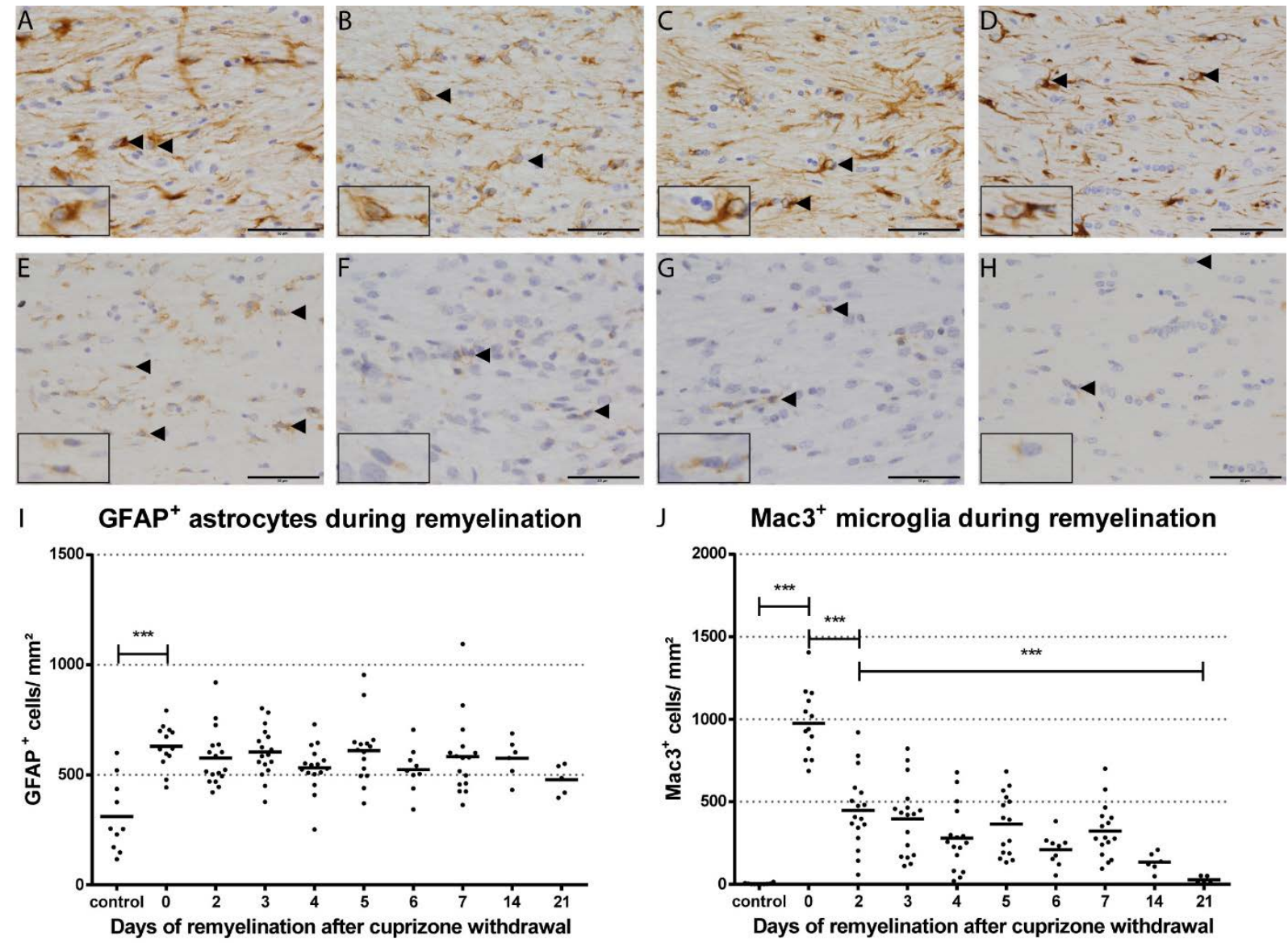

Figure 7: Microgliosis was declining during remyelination while astrogliosis sustained over time.

The numbers of reactive astrocytes (GFAP ${ }^{+}$cells) were constant during early remyelination (A-D, I) (mean; $n=5-17$; Kruskal Wallis test, Dunn's multiple comparison test; $\left.{ }^{* *} p<0.001\right)$. The number of activated/ $\mathrm{Mac}^{+}$microglia declined during early remyelination (F-H) in comparison to six weeks of demyelination (E). Microglial cells were activated after demyelination. The number of activated microglia was already significantly reduced two days after cuprizone withdrawal and almost absent after three weeks of remyelination ( $\mathbf{J}$ ) (mean; $\mathrm{n}=5-17$; one-way ANOVA, Bonferroni's multiple comparison test; $\left.{ }^{* \star} p<0.001\right)$. Representative images of medial corpus callosum of cuprizone-treated mice (A, E) and of mice after two (B, F), four $(\mathbf{C}, \mathbf{G})$ and seven $(\mathbf{D}, \mathbf{H})$ days of remyelination were taken at $400 x$ original magnification. Immunohistochemistry was performed for GFAP (A-D), and Mac3 $(\mathrm{E}-\mathrm{H})$ (scale bar $=50 \mu \mathrm{m})$.

\subsubsection{Expression of TGF $\beta$ and TNF $\alpha$ was upregulated during demyelination and remyelination}

Inflammatory cytokines might be secreted by microglia and/ or astrocytes and could thereby modify axonal injury in the cuprizone mouse model. Therefore, the expression of the inflammatory cytokines TGF $\beta$, TNF $\alpha$ and IL- 6 was assessed with qPCR from freshly isolated corpus callosum of cuprizone-treated mice. 
A

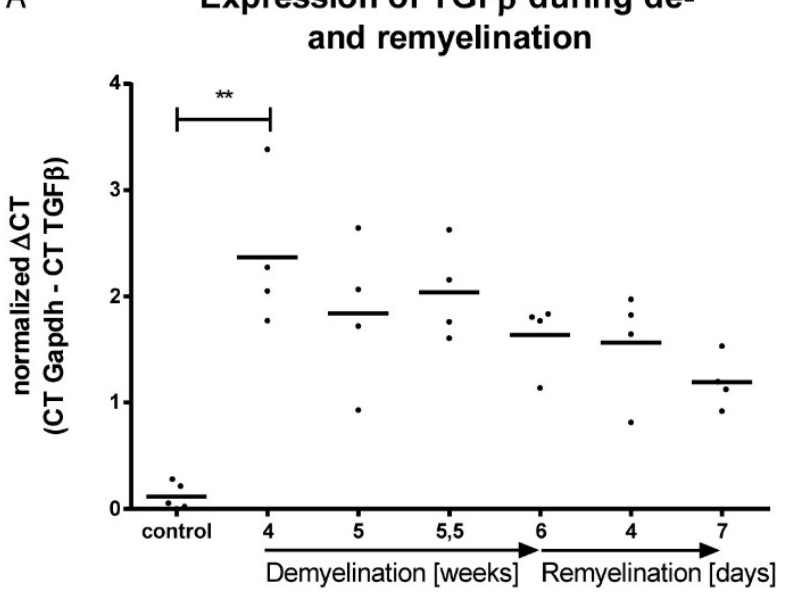

C

Expression of IL-6 during deand remyelination

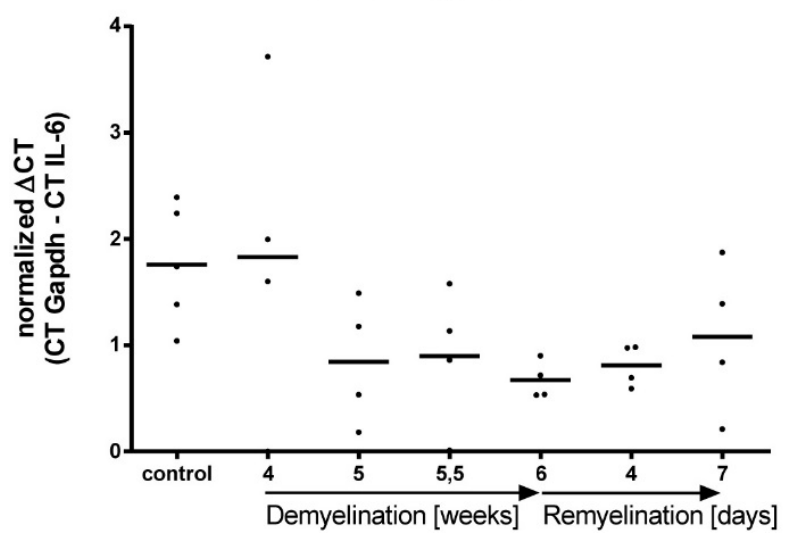

B

and remyelination

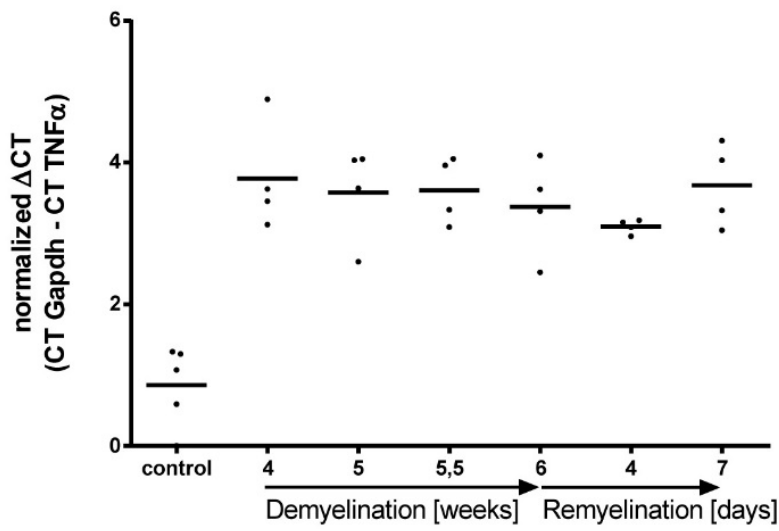

Figure 8: Expression of TGF $\beta$ and TNFa was upregulated during demyelination and remyelination.

The expression of IL-6, TGF $\beta$ and TNF $\alpha$ during de- and remyelination was analyzed in the cuprizone mouse model by qPCR. The $\triangle C T$ values (CT housekeeping gene (Gapdh) - CT gene of interest) were normalized to the lowest value measured. The housekeeping gene was constantly expressed in all samples (A-C). TGF $\beta$ was significantly upregulated by cuprizone treatment and was not significantly modified after withdrawal of cuprizone (A) (mean \pm SEM; $n=4-5$; Kruskal Wallis test, Dunn's multiple comparison test; ${ }^{*} \mathrm{p}<0.01$ ). TNFa was upregulated during cuprizone treatment which did not change after cuprizone withdrawal (B). No significant regulation of IL-6 was observed (C).

Expression of TGF $\beta$ was significantly upregulated after four weeks of cuprizone ingestion and was not significantly reduced during remyelination. TNFa was upregulated after four weeks and continuously expressed during demyelination and remyelination. The expression of IL-6 was not significantly modified during de- and remyelination (Fig. $8 A-C)$. 


\subsubsection{The percentage of myelinated damaged axons increased during remyelination}

To investigate the association of the myelin sheath with axonal spheroids a comprehensive confocal microscopy investigation was performed. The double immunostaining of APP and MBP revealed three subpopulations of $\mathrm{APP}^{+}$spheroids in the corpus callosum of cuprizone-treated mice (Fig. 9A-E): un- or demyelinated spheroids (mean: 68.2\%), partially myelinated spheroids (mean: $25.1 \%$ ) and myelinated spheroids (mean: 6.7\%). The partially myelinated subpopulation of $\mathrm{APP}^{+}$spheroids remained almost unchanged after the first week of remyelination (mean: 27.8\%), whereas the subpopulation of un- or demyelinated $\mathrm{APP}^{+}$spheroids decreased (mean: 47.2\%). Remarkably, the percentage of myelinated $\mathrm{APP}^{+}$spheroids increased significantly after one week of recovery (mean: $25 \%$ ).

Figure 9: The percentage of myelinated damaged axons increased during remyelination.

The relation of axonal damage and remyelination was evaluated in detail by double immunohistochemistry for APP and MBP. APP ${ }^{+}$axonal spheroids found in the corpus callosum of mice after demyelination and during remyelination could be divided into three different subpopulations: un- or demyelinated, partially myelinated and myelinated APP ${ }^{+}$ spheroids. APP ${ }^{+}$spheroids were analyzed in 20-30 randomly selected visual fields with at least one $\mathrm{APP}^{+}$spheroid ( $\sim 50$ spheroids) in the corpus callosum of mice by confocal microscopy (A-C). Spheroids partially or completely wrapped with myelin were found in the corpus callosum of mice after demyelination. After one week of remyelination the number of myelinated $\mathrm{APP}^{+}$spheroids increased significantly. Panel $\mathrm{E}$ shows the myelinated spheroids after six weeks of cuprizone ingestion and after one week of remyelination in more detail. Each dot represents the percentage of myelinated APP ${ }^{+}$ spheroids in the corpus callosum of an individual mouse. (D, E) (mean \pm SEM; $n=3-7$; unpaired $\mathrm{t}$ test; $\left.{ }^{* \star *} \mathrm{p}<0.001\right)$. Representative images of the subpopulations of $\mathrm{APP}^{+}$ spheroids were taken by confocal microscopy at 400x original magnification with $7 \%$ zoom (scale bar $=15 \mu \mathrm{m})$. 

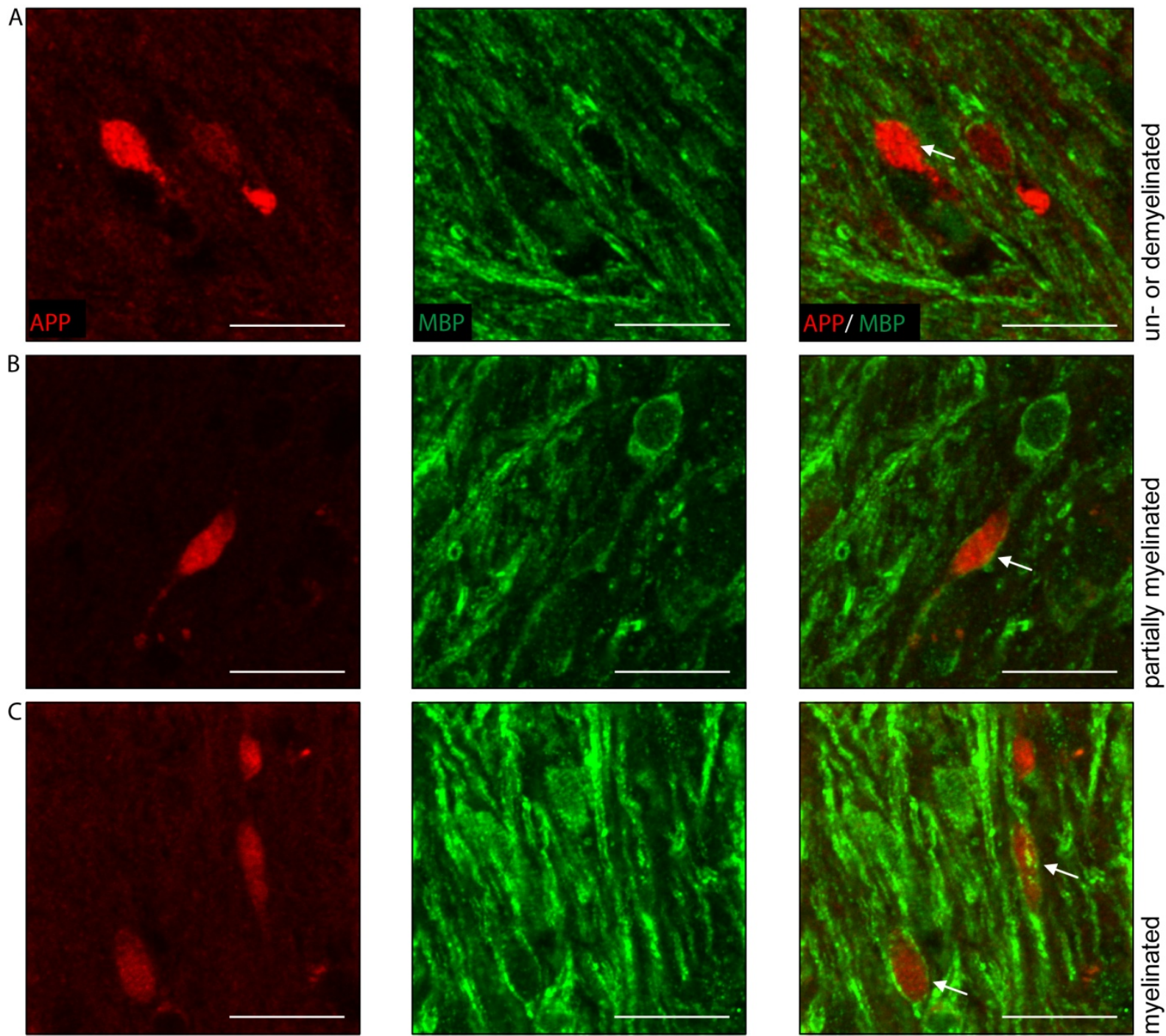

D Acute axonal damage in association with $E$ myelin during remyelination
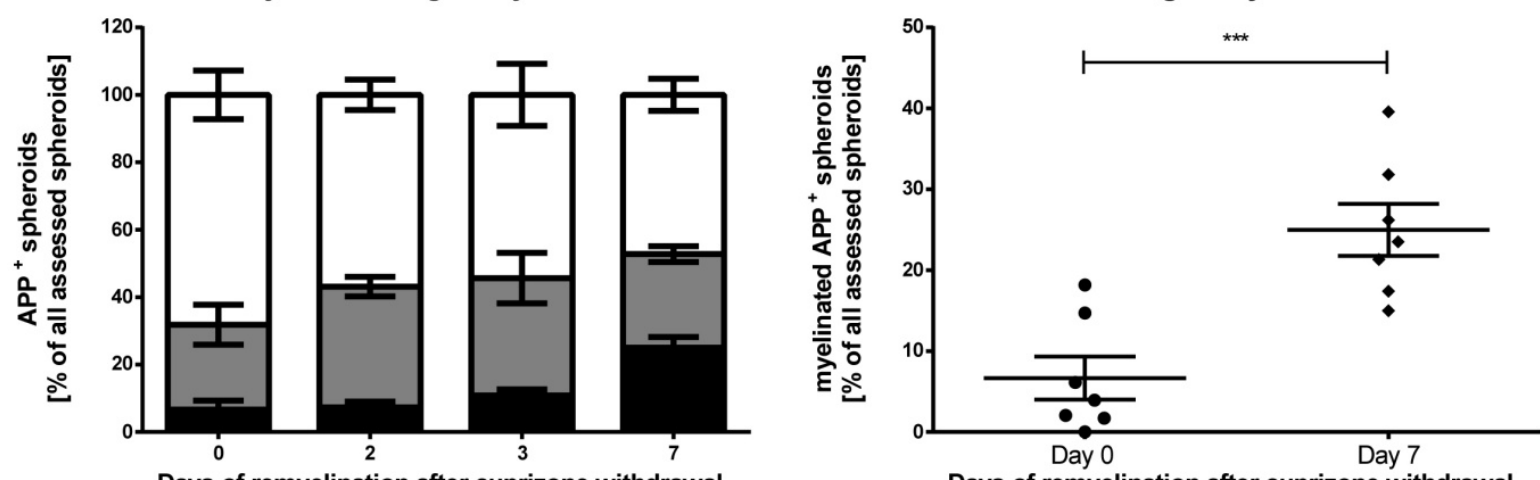

Days of remyelination after cuprizone withdrawal

Days of remyelination after cuprizone withdrawal

un- or demyelinated $\mathrm{APP}^{+}$spheroids

partially myelinated $\mathrm{APP}^{+}$spheroids

myelinated $\mathrm{APP}^{+}$spheroids 


\subsubsection{The corpus callosum exhibited large demyelinated areas without intact myelin sheaths after cuprizone challenge}

To assess whether the myelinated $\mathrm{APP}^{+}$spheroids could originate from myelinated damaged axons, ultrathin sagittal sections from corpus callosum of control and cuprizone-treated mice were analyzed by electron microscopy. The electron micrographs revealed large areas of demyelination after six weeks of cuprizone ingestion (Fig. 10B) compared to control (Fig. 10A). Furthermore, remyelinated axons and axons with increased mitochondrial content of damaged axons were found in the corpus callosum of cuprizone-treated mice (Fig. 10B-C).
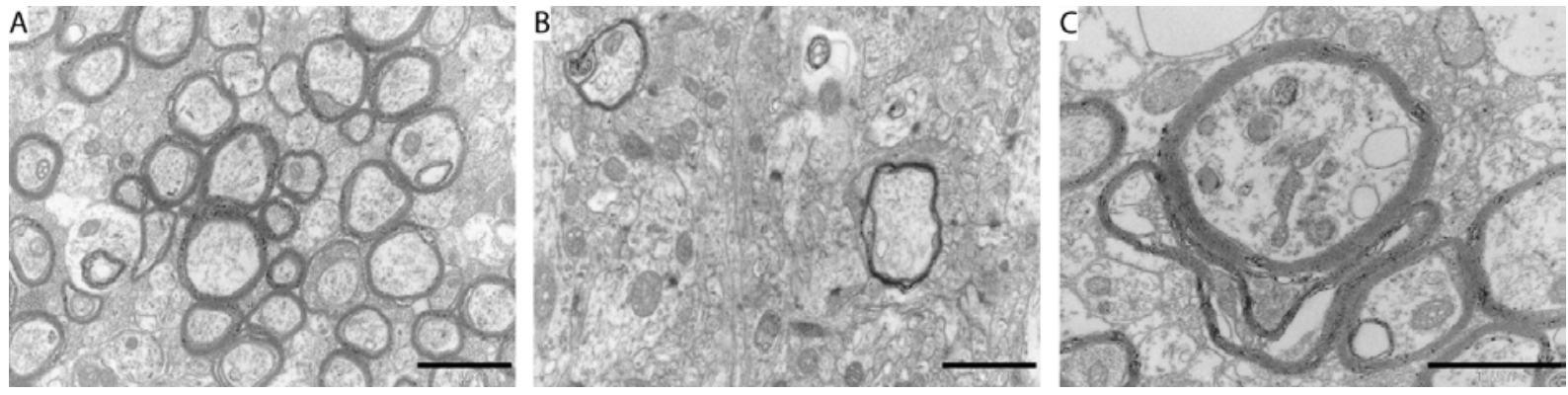

Figure 10: Electron microscopic analysis revealed disrupted myelin sheaths and increased mitochondrial content of damaged axons.

The corpus callosum of control mice $(\mathbf{A})$ and mice after cuprizone treatment $(\mathbf{B}, \mathbf{C})$ were analyzed by electron microscopy. The ultrastructural analysis revealed large areas of demyelination (B). Remyelinated axons (B) and myelinated axons with increased mitochondrial content of damaged axons were observed (C). Representative images of the medial corpus callosum of mice were shown $(\mathbf{A}-\mathbf{C})($ scale bar $=1 \mu \mathrm{m})$.

\subsection{7 (Re)myelinated damaged axons were observed in early remyelinating early active MS lesions}

Biopsy tissue of four MS patients was evaluated to determine whether myelinated $\mathrm{APP}^{+}$spheroids were present in early remyelinating early active MS lesions. The cases exhibited typical characteristics of MS lesions: focal inflammation, demyelination and tissue damage with preserved axons and oligodendrocytes. The lesions exhibited a contiguous demyelinating area with ongoing early remyelination. Early remyelination was determined by evaluation of the LFB-PAS staining and the immunoreactivity for myelin proteins PLP, CNPase and MBP (Fig. 11B-F). Furthermore the NogoA immunohistochemistry revealed the presence of oligodendrocytes (Fig. 11G). For investigation of the specimens, which were double labeled for APP and MBP, ten randomly selected visual fields with at least one $\mathrm{APP}^{+}$spheroid were analyzed by confocal microscopy, which showed many myelinated $\mathrm{APP}^{+}$spheroids in all investigated cases (Fig. 11A). 

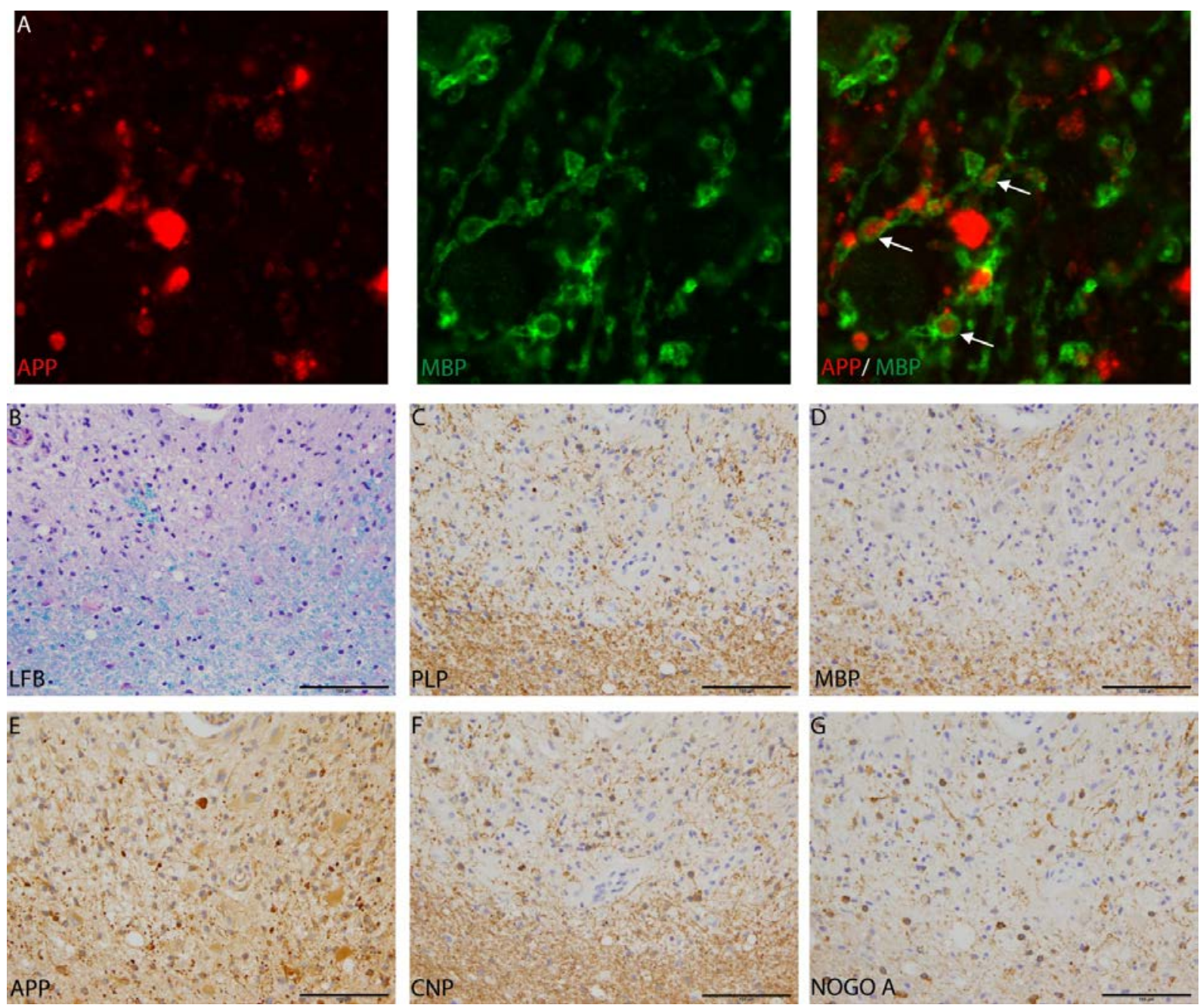

Figure 11: Myelinated $\mathrm{APP}^{+}$spheroids were observed in early remyelinating early active MS lesions.

Representative confocal image of myelinated APP ${ }^{+}$spheroids (arrows) in an early active MS lesion is shown in A (magnification $=400 x+7 \%$ zoom). The depicted MS lesion was an early active remyelinating lesion with Pattern II characteristics according to criteria described by Lucchinetti et al., 2000. The lesion border showed active demyelination while the lesion center revealed thin myelin sheaths characteristic for remyelination. This was also supported by many visible $\operatorname{Nogo}^{+}$mature oligodendrocytes (B-D, F, G). Numerous $\mathrm{APP}^{+}$spheroids were apparent in the lesion (E). Representative and descriptive images (B-G) of the depicted MS lesion were taken at 200x original magnification (scale bar $=100 \mu \mathrm{m})$.

\subsubsection{Late remyelinated MS lesions exhibited less acutely damaged axons than chronic inactive MS lesions}

The extent of acute axonal damage (APP) in chronic inactive and late remyelinated MS lesions (so called shadow plaques) was evaluated to assess whether remyelination was beneficial for axonal health in the long term. The chronic inactive MS 
lesions without signs of myelination showed significantly more ongoing acute axonal damage than remyelinated MS lesions (Fig. 12).

Acute axonal damage in chronic inactive and remyelinated MS lesions

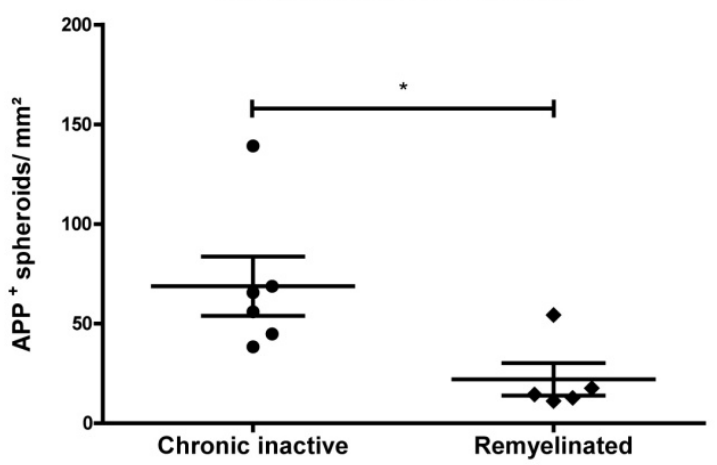

Figure 12: Late remyelinated MS lesions exhibited less $\mathrm{APP}^{+}$spheroids than chronic inactive MS lesions.

Acute axonal damage was evaluated by APP immunohistochemistry of six chronic inactive and five late remyelinated MS lesions (shadow plaques). Chronic inactive lesions revealed more $\mathrm{APP}^{+}$spheroids than remyelinated lesions (mean $\pm \mathrm{SEM} ; \mathrm{n}=5-6$; Mann Whitney test; $\left.{ }^{*} p<0.05\right)$.

\subsubsection{Colocalization of the paranodal protein Caspr with APP $^{+}$spheroids in the cuprizone mouse model}

Sections from mice after cuprizone withdrawal were double immunostained for Caspr, a paranodal protein, and APP to investigate whether remyelination and the formation of new nodes of Ranvier might have an impact on axonal vulnerability during remyelination. Caspr colocalized with $60-75 \%$ of $\mathrm{APP}^{+}$spheroids in the corpus callosum of mice after cuprizone diet cessation and was present as vesicle-like structures as evaluated by confocal microscopy (Fig. 13A, B). 

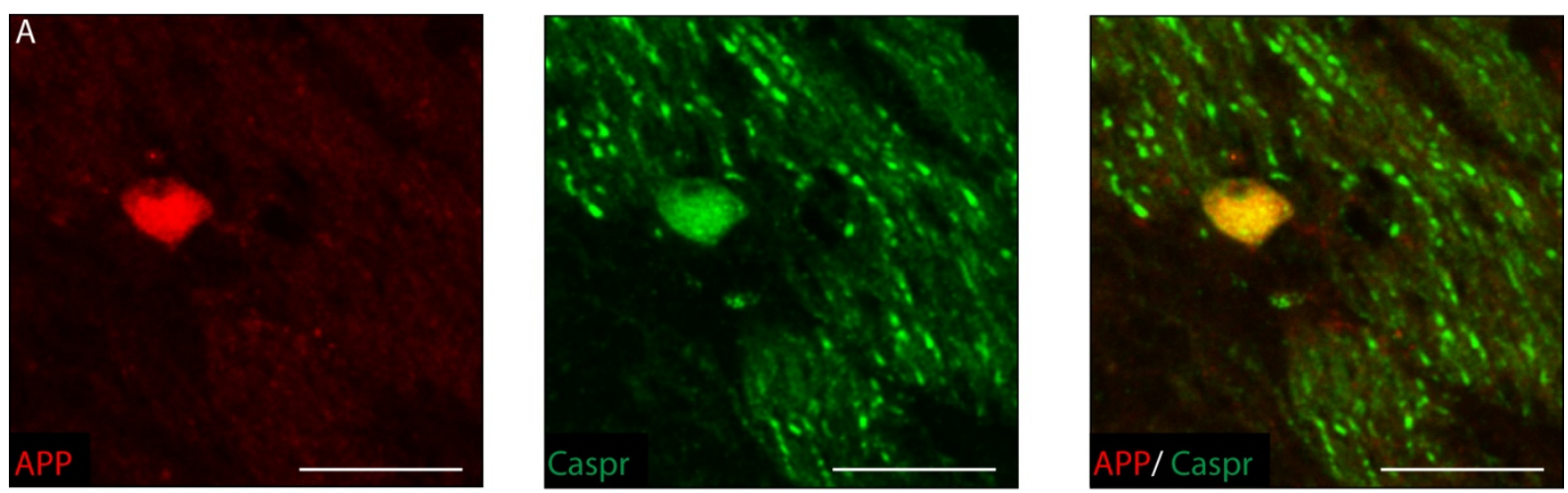

B Accumulation of Caspr in and around APP spheroids

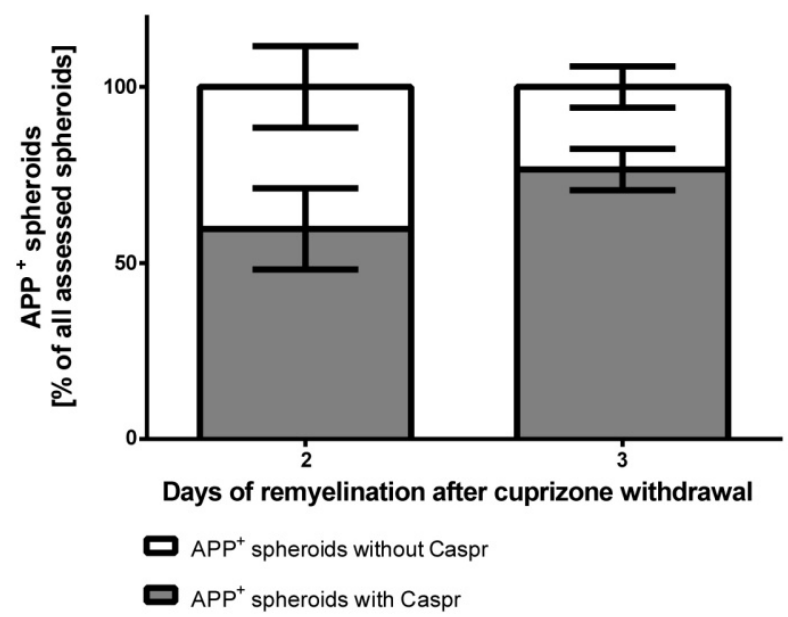

Figure 13: The paranodal protein Caspr was colocalized with $\mathrm{APP}^{+}$spheroids in the cuprizone mouse model.

The association of Caspr with acute axonal damage was analyzed by double immunohistochemistry for APP and Caspr. APP ${ }^{+}$spheroids were analyzed in 30 randomly selected visual fields with at least one $\mathrm{APP}^{+}$spheroid ( $\sim 50$ spheroids) in the corpus callosum of mice by confocal microscopy $(\mathbf{A}, \mathbf{B})$. Approximately $60-75 \%$ of the $\mathrm{APP}^{+}$ spheroids assessed were colocalized with the paranodal protein Caspr during early remyelination $(\mathbf{A}, \mathbf{B})$ (mean $\pm \mathrm{SEM} ; \mathrm{n}=3$ ). Representative confocal image was taken at $400 x$ original magnification with $7 \%$ zoom $(A)$ (scale bar $=15 \mu \mathrm{m})$.

\subsubsection{Cell processes of reactive astrocytes were found in the proximity of damaged axons}

To further characterize the acute axonal damage in our model, sections from mice after cuprizone withdrawal were double immunostained for APP and GFAP and investigated by confocal microscopy. $\mathrm{APP}^{+}$spheroids were surrounded with the cell processes of reactive astrocytes in up to $80 \%$ of all $\mathrm{APP}^{+}$spheroids assessed during early remyelination (Fig. 14A, B). 

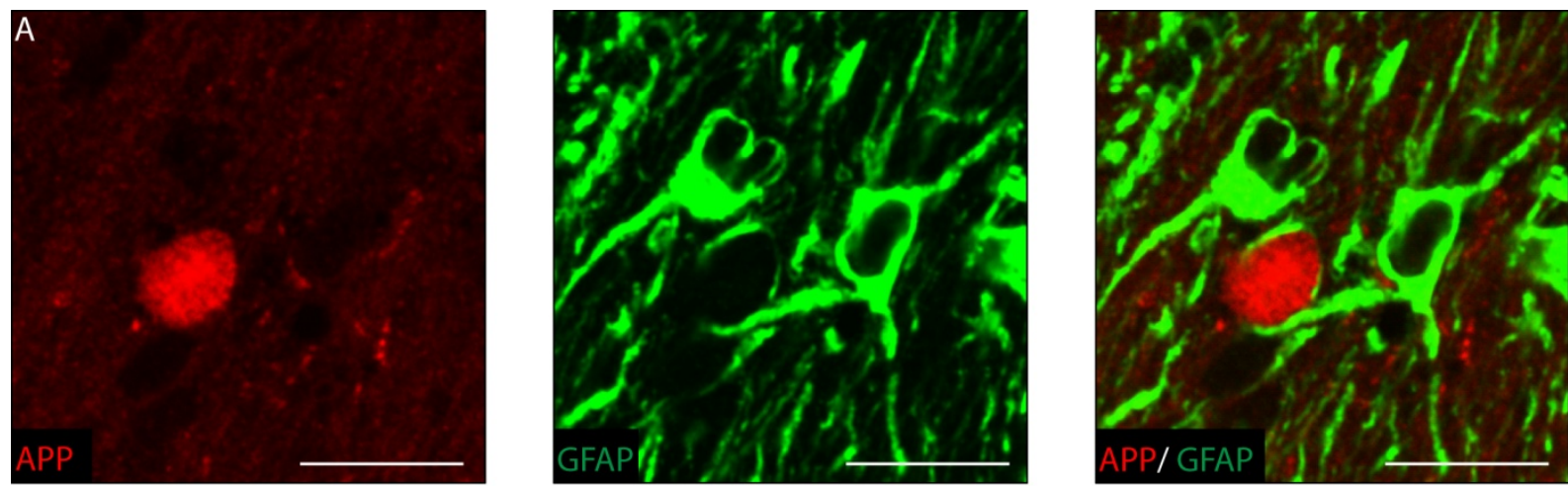

B Cell processes of $\mathrm{GFAP}^{+}$astrocytes in the proximity of $\mathrm{APP}^{+}$spheroids

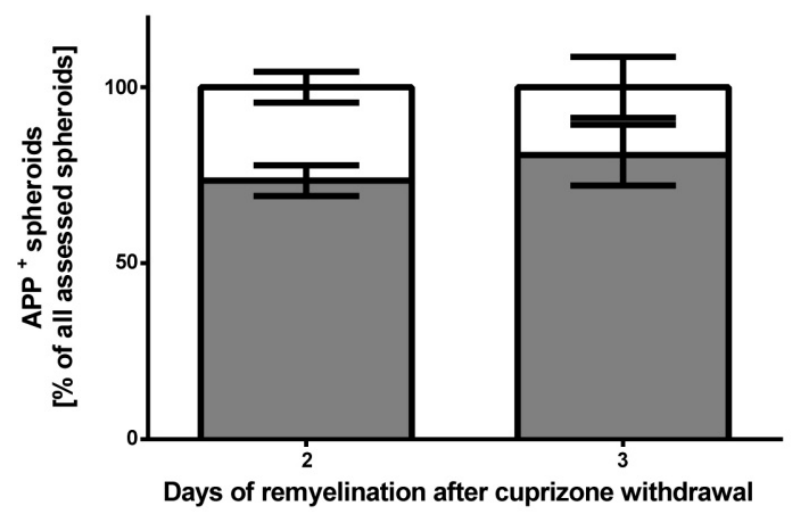

$\mathrm{APP}^{+}$spheroids without $\mathrm{GFAP}^{+}$cell processes of reactive astrocytes in the proximity

$\mathrm{APP}^{+}$spheroids surrounded by $\mathrm{GFAP}^{+}$cell processes of reactive astrocytes

Figure 14: $\mathrm{APP}^{+}$spheroids were surrounded by cell processes of reactive astrocytes in the cuprizone mouse model.

Immunoreactivity for GFAP showed that cell processes of reactive astrocytes were in the close proximity to $\mathrm{APP}^{+}$spheroids in up to $80 \%$ of all $\mathrm{APP}^{+}$spheroids assessed $(\mathbf{A}, \mathbf{B})$ (mean $\pm \mathrm{SEM} ; \mathrm{n}=3$ ). $\mathrm{APP}^{+}$spheroids were analyzed in 30 randomly selected visual fields with at least one $\mathrm{APP}^{+}$spheroid ( $\sim 50$ spheroids) in the corpus callosum of mice by confocal microscopy (A, B). Representative confocal image was taken at $400 x$ original magnification with $7 \%$ zoom $(\mathbf{A})$ (scale bar $=15 \mu \mathrm{m})$.

\subsection{Effects of ion channel inhibition on acute axonal damage during demyelination and remyelination}

The current study showed an accumulation of Caspr at sites of acute axonal injury, which might occur as a result of increased formation of nodes of Ranvier. Therefore to analyze the role of ion channels and to assess whether the ion channel redistribution during remyelination has an impact on axonal preservation ion channel inhibitors were administered during remyelination. After six weeks of cuprizone challenge mice were treated either with the unspecific $K_{v}$ channel inhibitor 4-aminopyridine (twice a day, $100 \mu \mathrm{g} /$ mouse, s.c.), the ASIC inhibitor amiloride (once a day, $200 \mu \mathrm{g} /$ mouse, i.p.) or vehicle (PBS s.c. or i.p.) (Fig. 15A). In another approach 4-aminopyridine was administered a few days preceding cuprizone withdrawal, to achieve higher levels of the 
drug at the onset of remyelination (Fig. 15B). The corpus callosum (CC) was divided into the medial and lateral parts for histological evaluation, because of the regional heterogeneity of the cuprizone-induced demyelination (Fig. 15C).
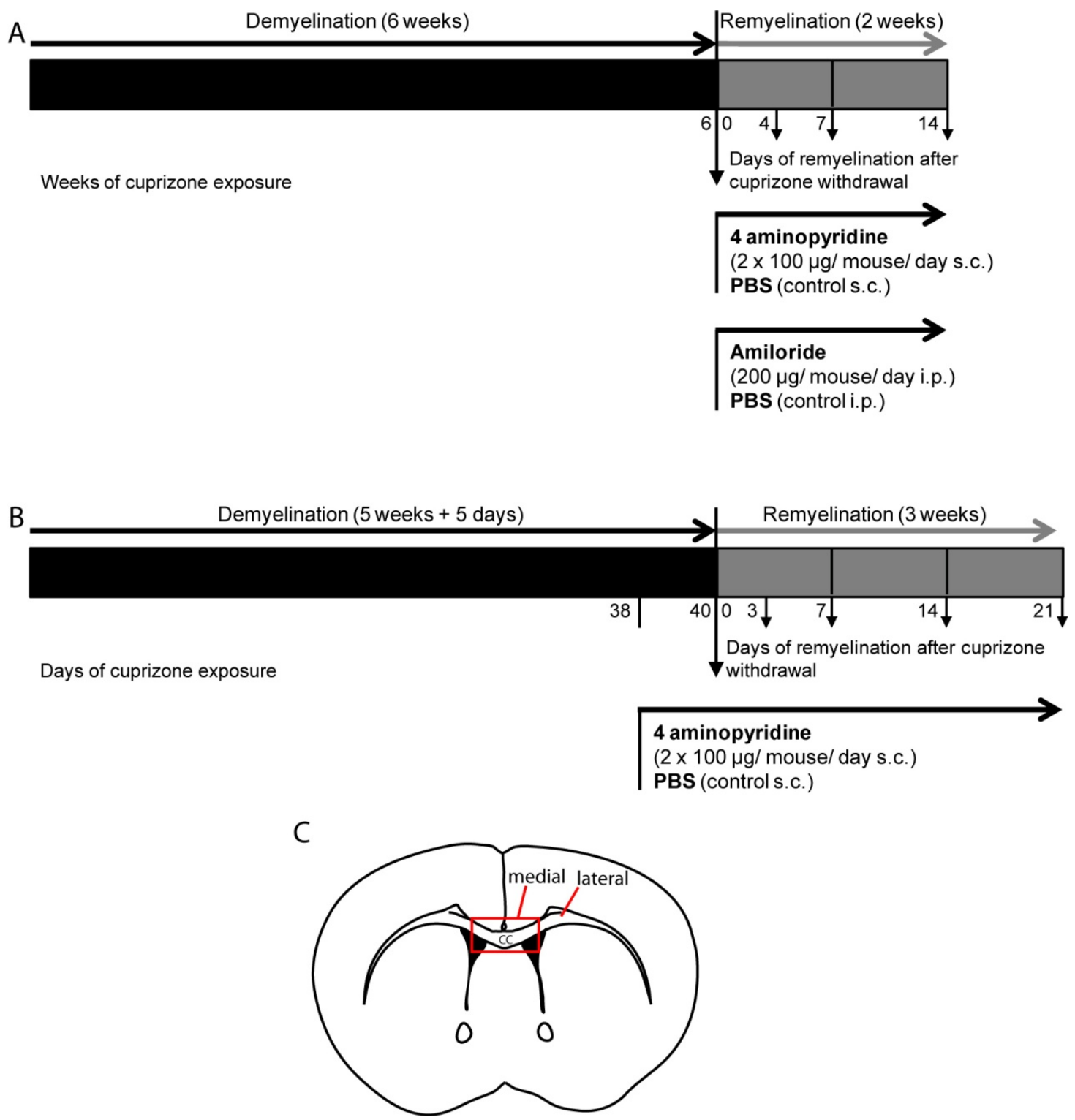

Figure 15: Design of the cuprizone experiments to investigate the effects of ion channel inhibition on acute axonal damage during remyelination.

Seven to eight week old male C57BL/6J mice were fed for six weeks with a $0.25 \%$ cuprizone diet to induce demyelination. The unspecific $\mathrm{K}_{v}$ channel inhibitor 4aminopyridine, the ASIC inhibitor amiloride or PBS were administered for two weeks during remyelination. After four, seven or 14 days brains of mice were harvested and analyzed by histology (Exp. 1) (A). In a second approach 4-aminopyridine was applied during the last days of cuprizone treatment. Brains were harvested and analyzed at day 38 and 40 of cuprizone treatment and at day three, seven, 14 or 21 of remyelination (Exp. 2) (B). The medial and lateral parts of the corpus callosum were analyzed separately as indicated (C). 


\subsubsection{Acute axonal damage was not influenced by $K_{v}$ channel inhibition during remyelination.}

The inhibition of potassium channels might reduce the energy demand, i.e. ATP consumption, of demyelinated axons, which is necessary to maintain the ion homeostasis, and might thereby preserve the axonal integrity and reduce the axonal damage. To assess whether potassium channel inhibition by 4-aminopyridine could reduce the acute axonal damage, specimens of mice after cuprizone withdrawal were

A Influence of $K_{V}$ channel blockage on acute axonal damage in the medial $\mathrm{CC}$ during remyelination (Exp.1)

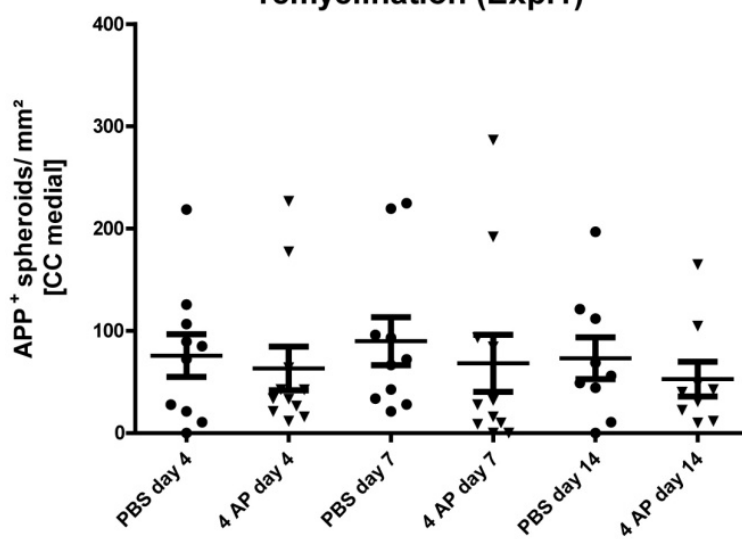

Days of remyelination after cuprizone withdrawal

C Influence of $K_{V}$ channel blockage on acute axonal damage in the medial CC during early and late remyelination (Exp.2)

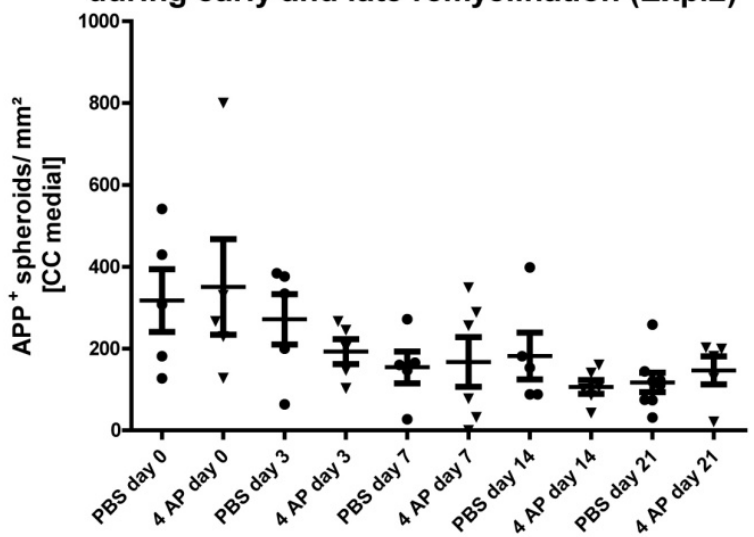

Days of remyelination after cuprizone withdrawal
B Influence of $K_{V}$ channel blockage on acute axonal damage in the lateral $\mathrm{CC}$ during

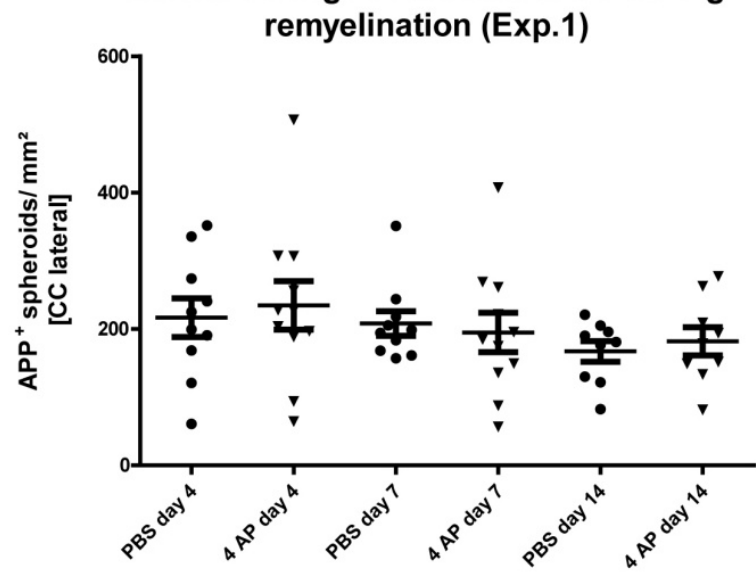

Days of remyelination after cuprizone withdrawal

D Influence of $K_{V}$ channel blockage on acute axonal damage in the lateral CC during early and late remyelination (Exp.2)

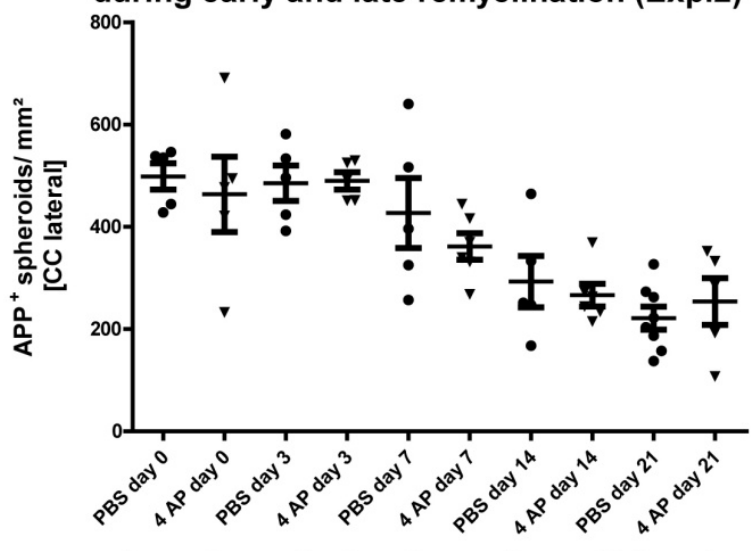

Days of remyelination after cuprizone withdrawal

Figure 16: $K_{v}$ channel inhibition did not influence the acute axonal damage during remyelination.

The number of $\mathrm{APP}^{+}$spheroids in the medial $(\mathbf{A}, \mathbf{C})$ and in the lateral $(\mathbf{B}, \mathbf{D})$ corpus callosum of 4-aminopyridine-treated mice was comparable to the acute axonal damage in the corpus callosum of PBS-treated mice during remyelination in both experimental setups (Exp. 1: A, B) (Exp. 2: C, D) (mean \pm SEM; $n=5-11)$. 
immunostained for APP and evaluated. The acute axonal damage in the corpus callosum of 4-aminopyridine-treated animals was comparable to vehicle-treated animals (Exp. 1) (Fig. 16A, B). Acute axonal damage in the corpus callosum was also not influenced by 4-aminopyridine treatment started during the last days of cuprizone ingestion (Exp. 2) (Fig. 16C, D).

\subsection{2 $K_{V}$ channel inhibition did not reduce significantly the percentage of myelinated damaged axons}

The axonal damage in relation to remyelination was assessed in 4-aminopyridinetreated mice by immunostaining for APP and MBP to investigate whether the inhibition of voltage-gated potassium channels could influence the percentage of the myelinated spheroids (Exp.1). The 4-aminopyridine-treated group showed no significant reduction of the percentage of myelinated $\mathrm{APP}^{+}$spheroids after one week of recovery. Additionally, there was no difference between the two groups after two weeks of remyelination (Fig.17).

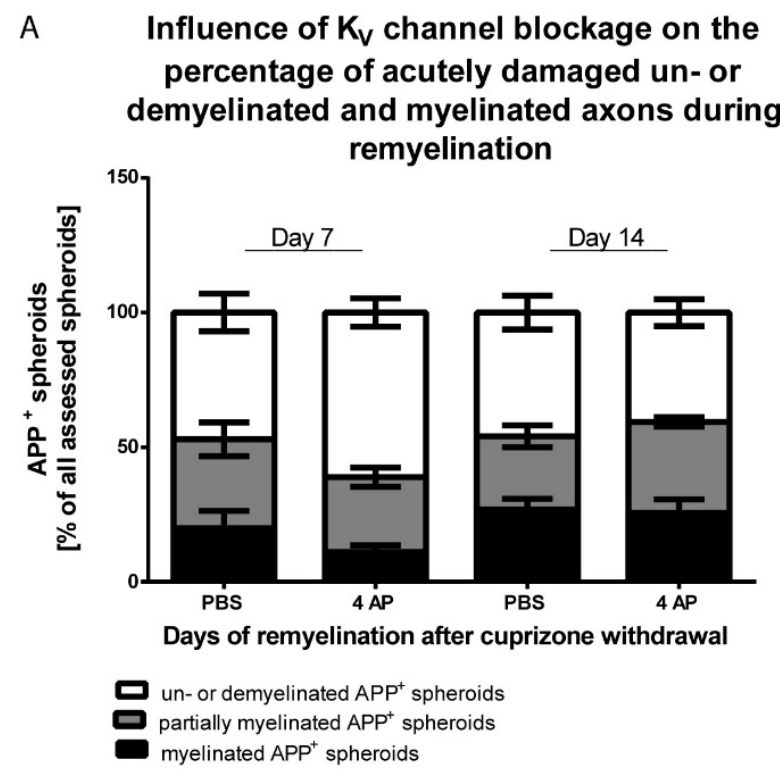

Figure 17: Mice treated with 4-aminopyridine exhibited no significant difference in the percentage of myelinated $\mathrm{APP}^{+}$spheroids compared to control.

After one week of recovery the percentage of myelinated $\mathrm{APP}^{+}$spheroids was not significantly different in the corpus callosum of 4-aminopyridine-treated mice compared to vehicle-treated mice (mean \pm SEM; $n=6$; unpaired t test, $p=0.2287$ ). Images of 20-30 randomly selected visual fields with at least one $\mathrm{APP}^{+}$spheroid ( $\sim 50$ spheroids/ corpus callosum) were analyzed by confocal microscopy. (400x original magnification with $7 \%$ zoom). 


\subsubsection{The number of reactive astrocytes was not influenced by $K_{v}$ channel inhibition}

Numbers of reactive astrocytes (GFAP ${ }^{+}$astrocytes) were evaluated after one and two weeks of recovery (Exp.1) to determine whether reactive astrocytes were modified by potassium channel inhibition. The sections of 4-aminopyridine-treated animals exhibited the same density of reactive astrocytes as the vehicle-treated animals. Furthermore, the medial and lateral parts of the corpus callosum showed a constant and similar level of astrogliosis over time in both groups (Fig. 18).

A

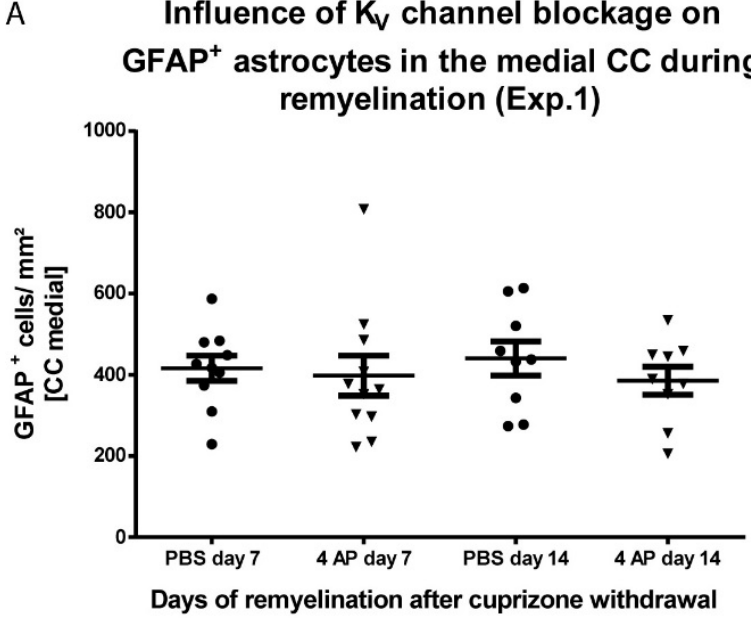

B Influence of $K_{V}$ channel blockage on GFAP $^{+}$astrocytes in the lateral CC during remyelination (Exp.1)

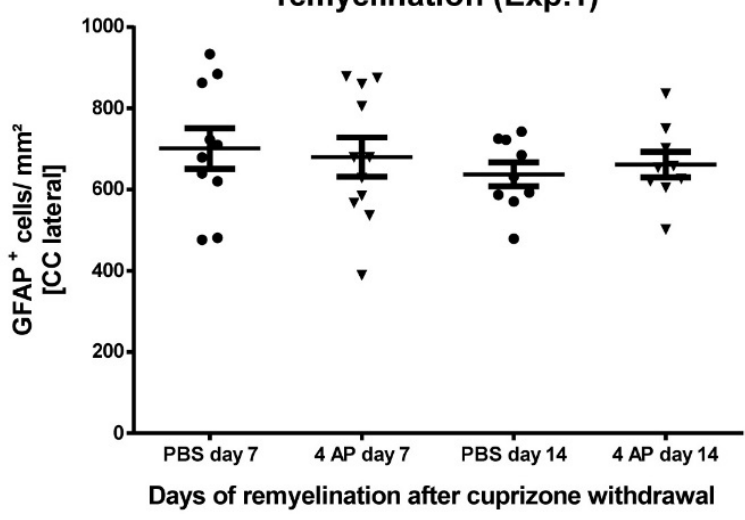

Figure 18: No difference in the number of reactive astrocytes by $K_{v}$ channel inhibition during remyelination.

The density of GFAP $^{+}$astrocytes in the medial (A) as well as in the lateral (B) corpus callosum of 4-aminopyridine-treated mice was comparable to the astrogliosis in the corpus callosum of PBS-treated mice during early and late remyelination (mean \pm SEM; $n$ $=9-11)$.

\subsubsection{Microgliosis was increased during remyelination by $K_{v}$ channel inhibition}

To examine whether diminished microgliosis could be associated with a reduced number of myelinated $\mathrm{APP}^{+}$spheroids, the number of activated $\mathrm{Mac}^{+}$microglia was evaluated after one and two weeks of remyelination (Exp.1). Mice-treated with 4aminopyridine showed an increase of $\mathrm{Mac}^{+}$microglial cells in the medial and lateral corpus callosum during remyelination (Fig. 19). 

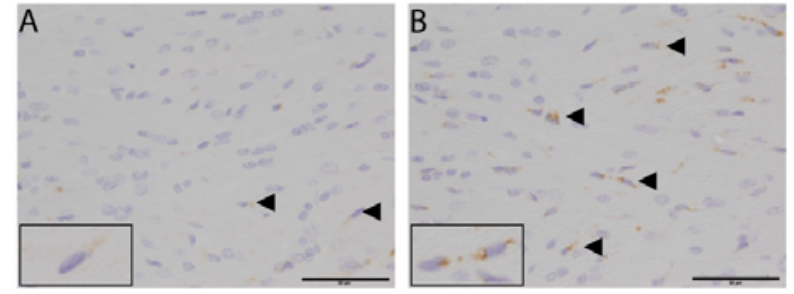

$\mathrm{E}$

Influence of $\mathrm{K}_{\mathrm{V}}$ channel blockage on

$\mathrm{Mac}^{+}$microglia in the medial $\mathrm{CC}$ during remyelination (Exp.1)

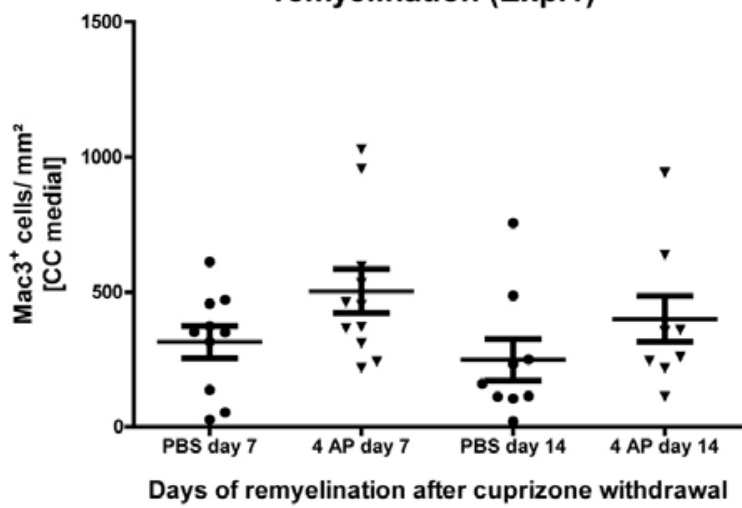

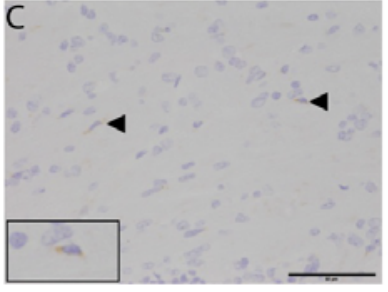

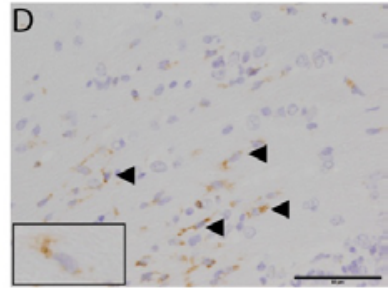

$\mathrm{F}$

Influence of $K_{v}$ channel blockage on $\mathrm{Mac}^{+}$microglia in the lateral CC during remyelination (Exp.1)

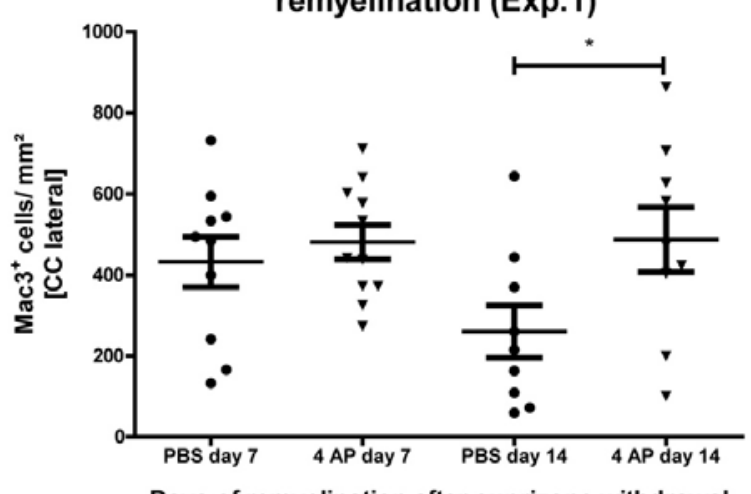

Days of remyelination after cuprizone withdrawal

Figure 19: Microgliosis was increased by $K_{V}$ channel inhibition during remyelination.

The number of activated microglia $\left(\mathrm{Mac}^{+}\right)$was increased in the medial $(\mathbf{B}, \mathbf{E})$ and lateral (D, F) corpus callosum of 4-aminopyridine-treated mice compared to the medial (A, E) and lateral (C, F) corpus callosum of PBS-treated animals (mean \pm SEM; $n=9-11$; Kruskal Wallis test, Dunn's multiple comparison test; $\left.{ }^{*} \mathrm{p}<0.05\right)$. Representative images of the medial and lateral corpus callosum at day 14 of remyelination were taken at $400 x$ original magnification (scale bar $=50 \mu \mathrm{m}$ ).

\subsubsection{The number of oligodendrocytes was accelerated in the medial corpus callosum by $\mathrm{K}_{\mathrm{V}}$ channel inhibition during remyelination.}

To evaluate the effect of potassium channel inhibition on oligodendrocytes in the cuprizone mouse model (Exp.1), the densities of $\mathrm{Olig}^{+}$and $\mathrm{NogoA}^{+}$oligodendrocytes were determined. The lateral corpus callosum revealed similar numbers of $\mathrm{Olig}^{+}$and Nogo $^{+}$oligodendrocytes during remyelination in both groups (Fig. 20F, H). In contrast, the numbers of the oligodendrocytes in the medial corpus callosum were increased after 14 days of remyelination by 4-aminopyridine administration (Fig. 20A-E, G). 

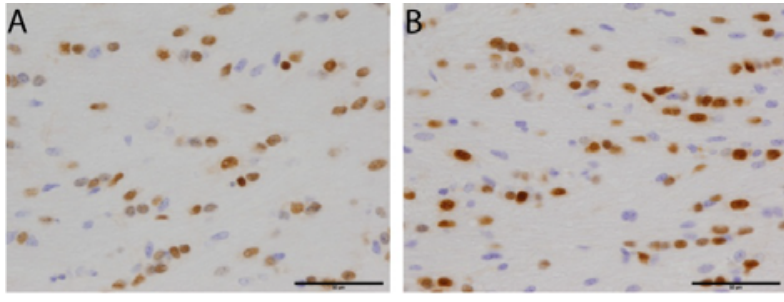

$\mathrm{E}$

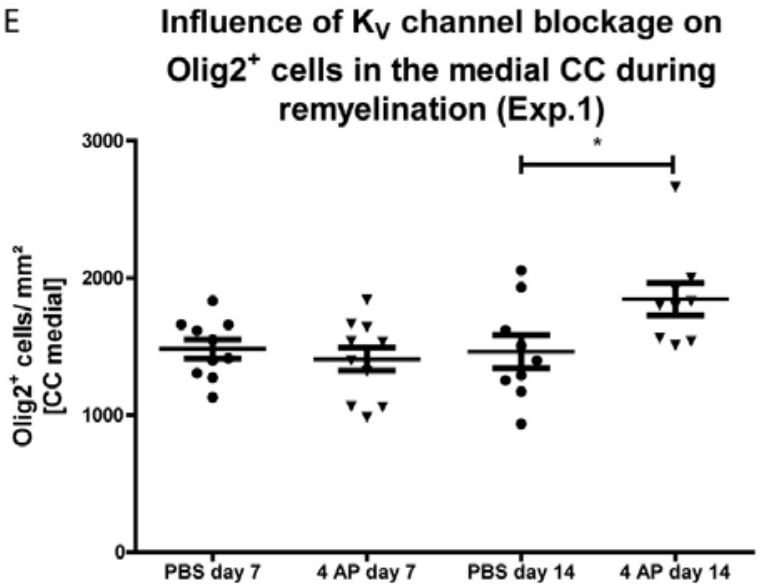

Days of remyelination after cuprizone withdrawal

G

Influence of $K_{V}$ channel blockage on

Nogo $\mathrm{A}^{+}$cells in the medial $\mathrm{CC}$ during remyelination (Exp.1)

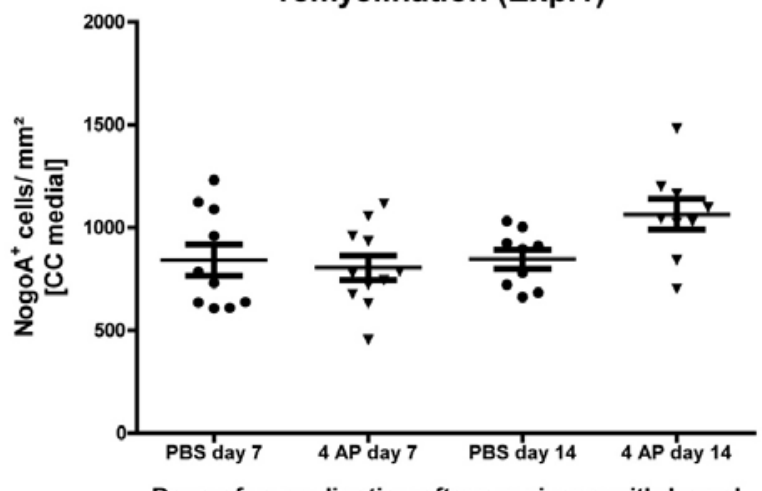

Days of remyelination after cuprizone withdrawal
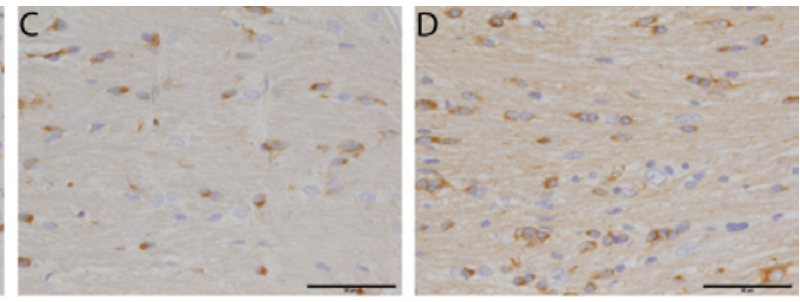

Influence of $K_{V}$ channel blockage on

Olig2 ${ }^{+}$cells in the lateral $\mathrm{CC}$ during remyelination (Exp.1)

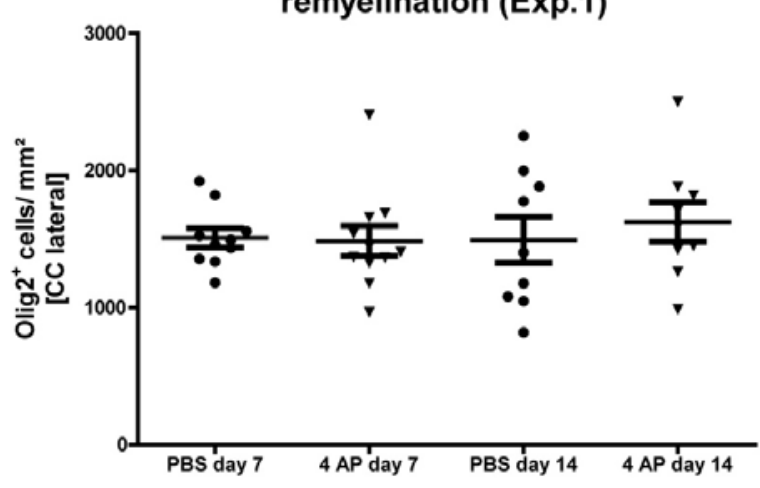

Days of remyelination after cuprizone withdrawal

$\mathrm{H}$ Influence of $\mathrm{K}_{\mathrm{V}}$ channel blockage on Nogo $\mathrm{A}^{+}$cells in the lateral $\mathrm{CC}$ during remyelination (Exp.1)

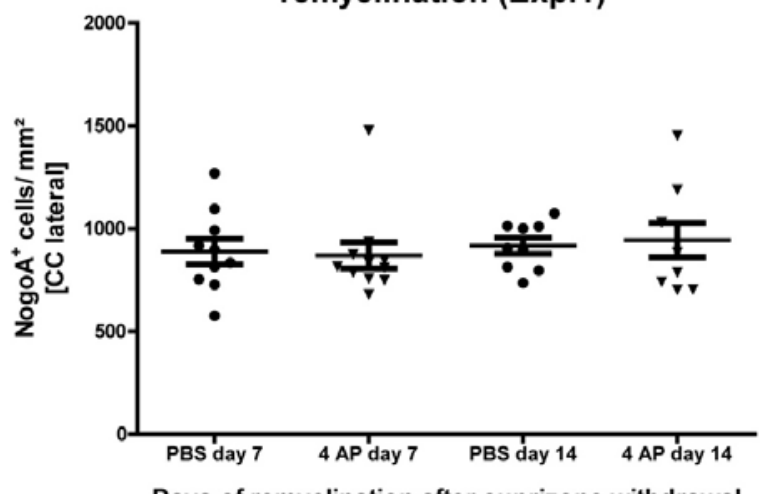

Days of remyelination after cuprizone withdrawal

Figure 20: The number of oligodendrocytes was accelerated in the medial corpus callosum by $\mathrm{K}_{\mathrm{v}}$ channel inhibition during remyelination.

The medial corpus callosum of 4-aminopyridine-treated animals (B, D, E, G) showed more Olig2 ${ }^{+}$and $\mathrm{NogoA}^{+}$oligodendrocytes than the medial corpus callosum of control animals (A, C, E, G) (mean \pm SEM; $n=9-11$; one-way ANOVA, Bonferroni's multiple comparison test; $\left.{ }^{*} p<0.05\right)$. The lateral corpus callosum of 4 -aminopyridine-treated animals was comparable to the lateral corpus callosum of control animals $(\mathbf{F}, \mathbf{H})$. Representative images of the medial corpus callosum were taken at 400x original magnification $(\mathbf{A}, \mathbf{B}=$ Olig2) $(\mathbf{C}, \mathbf{D}=$ NogoA) $($ scale bar $=50 \mu \mathrm{m})$. 


\subsubsection{ASIC inhibition did not modify acute axonal damage or astrogliosis, but modified microgliosis}

The ASIC inhibitor amiloride was administered during remyelination to test whether this ion channel inhibitor was able to improve axonal preservation in the cuprizone mouse model during remyelination (Exp.1). Application of the ASIC inhibitor amiloride during remyelination did neither have an effect on acute axonal damage nor on astrogliosis (Fig. 21A-D).

A Influence of ASIC inhibition on acute axonal damage in the medial $\mathrm{CC}$ during remyelination

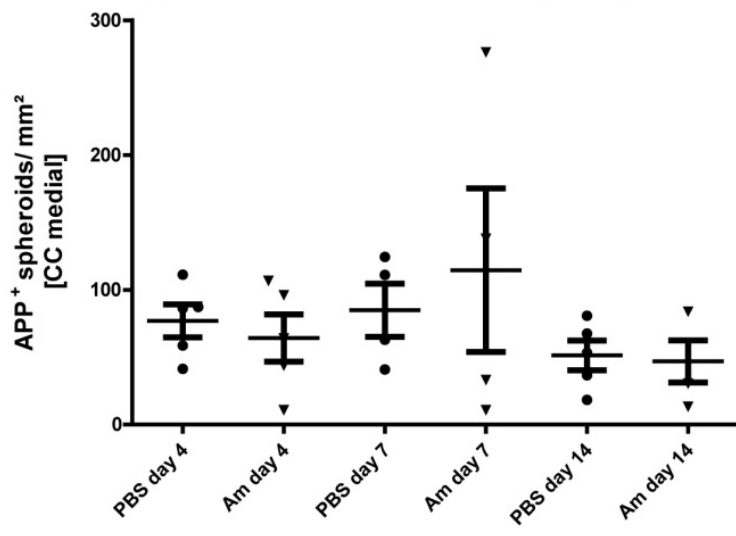

C

Days of remyelination after cuprizone withdrawal GFAP $^{+}$astrocytes in the medial CC during remyelination

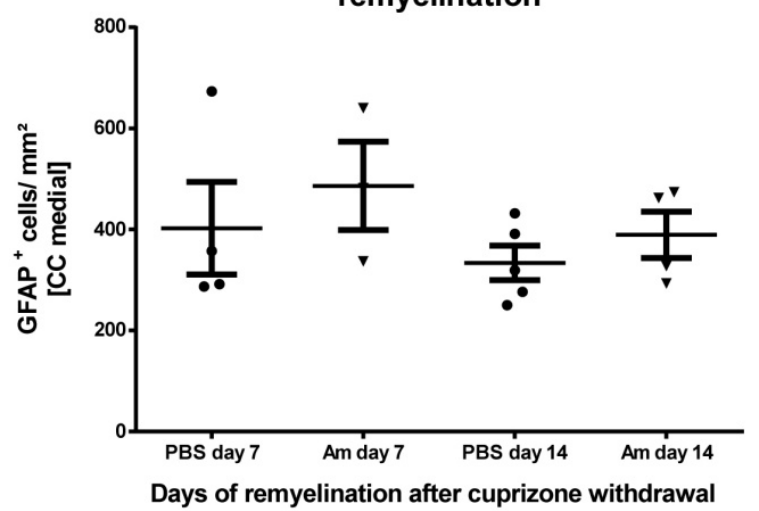

B Influence of ASIC inhibition on acute axonal damage in the lateral $\mathrm{CC}$ during remyelination

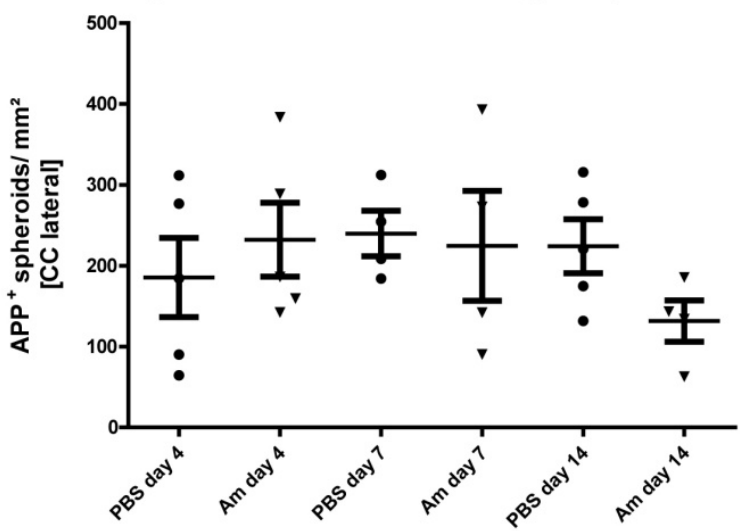

D

Days of remyelination after cuprizone withdrawal Influence of ASIC inhibition on

$\mathrm{GFAP}^{+}$astrocytes in the lateral CC during remyelination

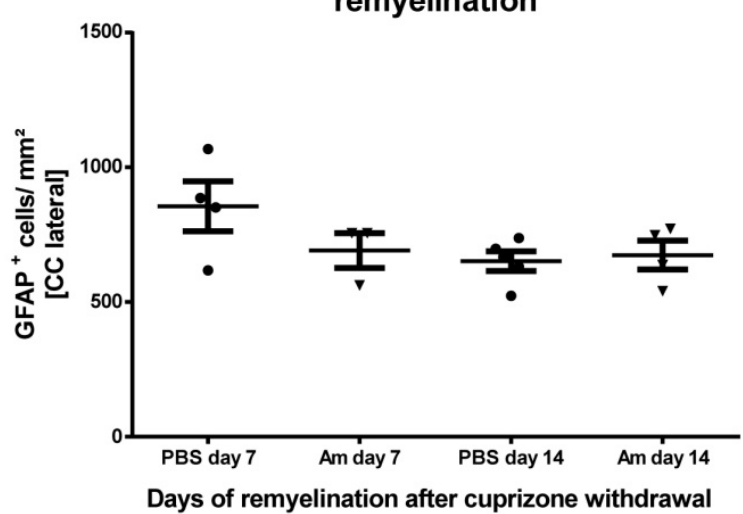

Figure 21: ASIC inhibition did neither modify acute axonal damage nor astrogliosis.

The numbers of $\mathrm{APP}^{+}$spheroids and reactive $\mathrm{GFAP}^{+}$astrocytes were determined in the medial $(\mathbf{A}, \mathbf{C})$ and lateral $(\mathbf{B}, \mathbf{D})$ corpus callosum of amiloride (Am) and PBS-treated mice. The evaluation of acute axonal damage (A, B) and astrogliosis (C, D) revealed no difference between the two groups (mean \pm SEM; $n=3-5$ ). 
However, ASIC channel inhibition appeared to modify the extent of microgliosis in the medial corpus callosum two weeks after cuprizone diet cessation ( $p=0.0635$ ) (Fig. 22).

$E$
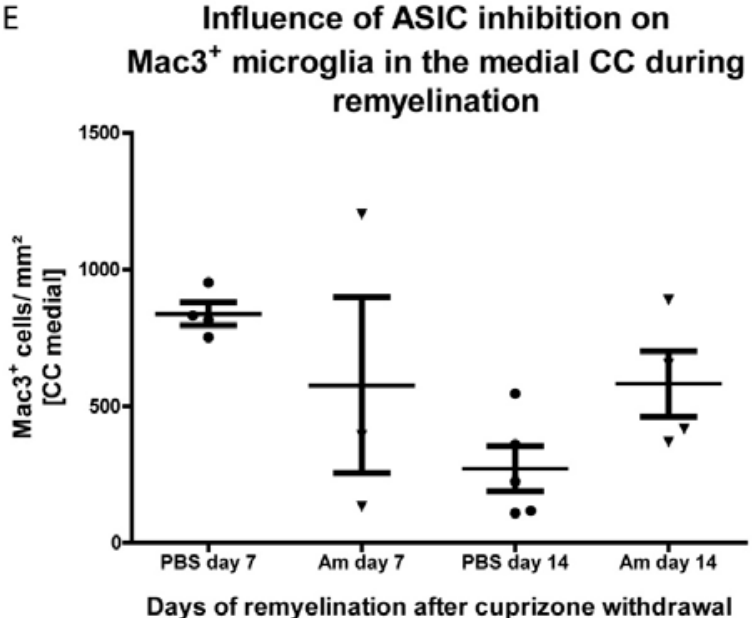

$\mathrm{F}$
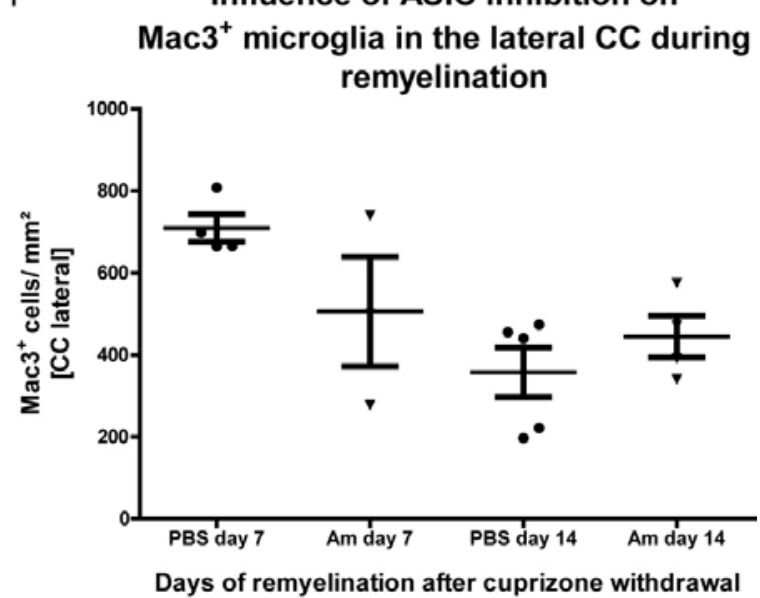

Figure 22: ASIC inhibition exhibited a tendency to accelerate the extent of microgliosis.

Activated microglia (Mac3 ${ }^{+}$cells) were determined in the medial $(\mathbf{A})$ and lateral (B) corpus callosum of amiloride (Am) and PBS-treated mice. Mice treated with amiloride appeared to accelerate the extent of microgliosis in the medial corpus callosum two weeks after cuprizone withdrawal (mean \pm SEM; $n=3-5$; Mann Whitney test; $p=0.0635$ ).

\subsubsection{The number of oligodendrocytes was not influenced by ASIC inhibition}

The inhibition of voltage-gated potassium channels resulted in increased numbers of oligodendrocytes two weeks after cuprizone diet cessation. Therefore other ion channel inhibitors such as amiloride might have the capability to modify the density of oligodendrocytes. Immunohistochemistry for Olig2 and p25 (mature oligodendrocytes) of amiloride and PBS-treated mice were evaluated (Exp.1). The numbers of Olig2 ${ }^{+}$ oligodendrocytes and $\mathrm{p}^{+} 5^{+}$mature oligodendrocytes were comparable between the amiloride and the vehicle-treated mice during remyelination (Fig. 23). 

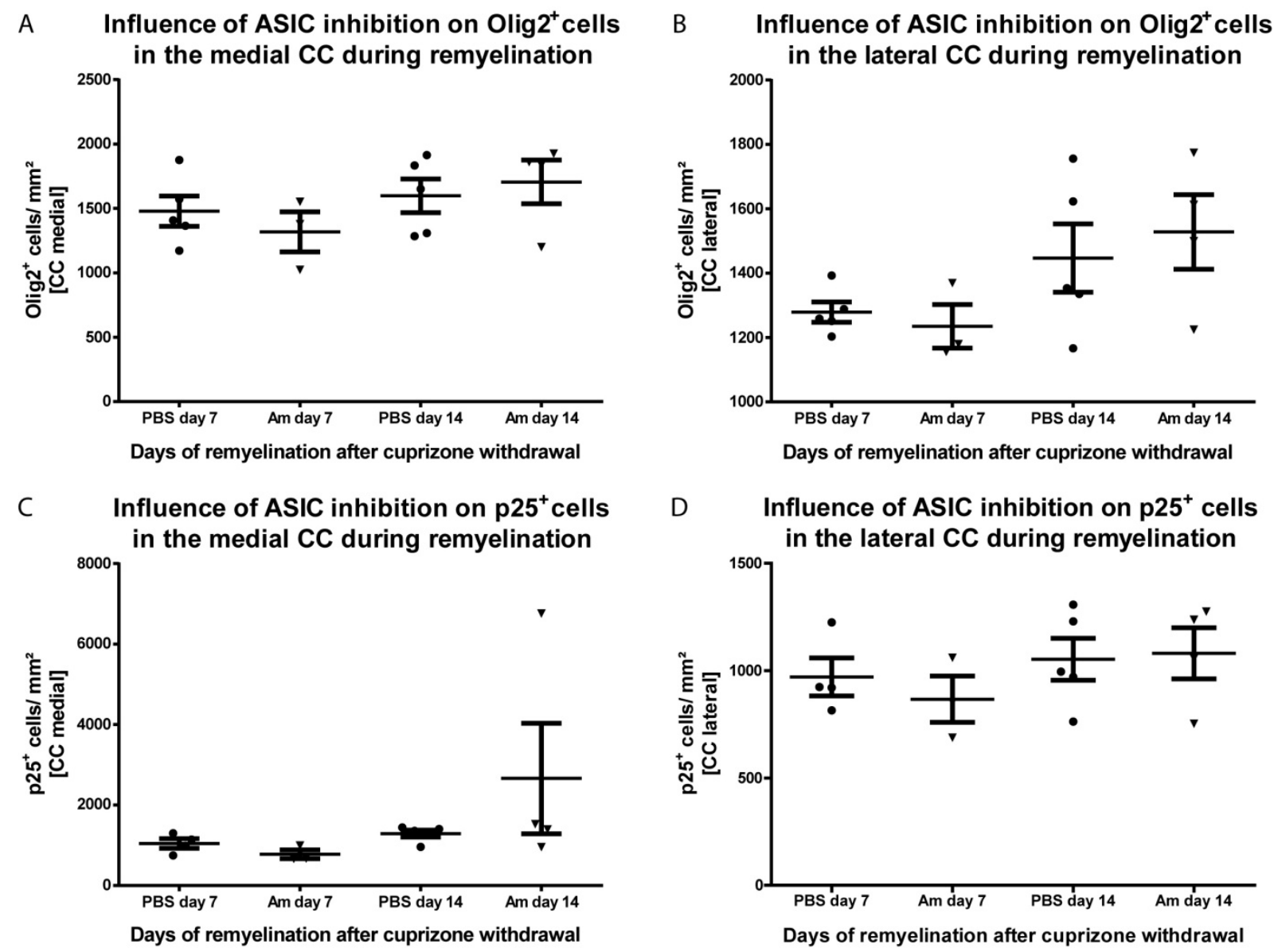

Figure 23: The number of oligodendrocytes was not influenced by ASIC inhibition.

The immunoreactivity for Olig2 (A, B) and p25 (C, D) revealed no difference of the number of oligodendrocytes in the corpus callosum of amiloride and PBS-treated animals (mean \pm SEM; $n=3-5$ ).

\subsubsection{Inhibition of $\mathrm{K}_{\mathrm{V}}$ channels did not alter the demyelinated area of the corpus callosum in the cuprizone mouse model}

The results regarding ion channel inhibition during remyelination revealed an increased number of oligodendrocytes, while the numbers of $\mathrm{APP}^{+}$spheroids were similar between mice treated with ion channel inhibitors and those treated with vehicle. The potential to modify the acute axonal damage and the numbers of oligodendrocytes was therefore determined during cuprizone-induced demyelination. After mice received cuprizone diet for three weeks, the ion channel inhibitors 4-aminopyridine and amiloride were administered during the following three weeks of cuprizone challenge. The acute axonal damage (Fig. 24) and the number of oligodendrocytes (Fig. 25) in the corpus callosum of 4-aminopyridine and amiloride-treated mice were not significantly different from controls. Mice treated with 4-aminopyridine or amiloride showed comparable demyelination of the corpus callosum at week six (Fig. 26). 
A Influence of ion channel inhibition on acute axonal damage in the medial CC during demyelination

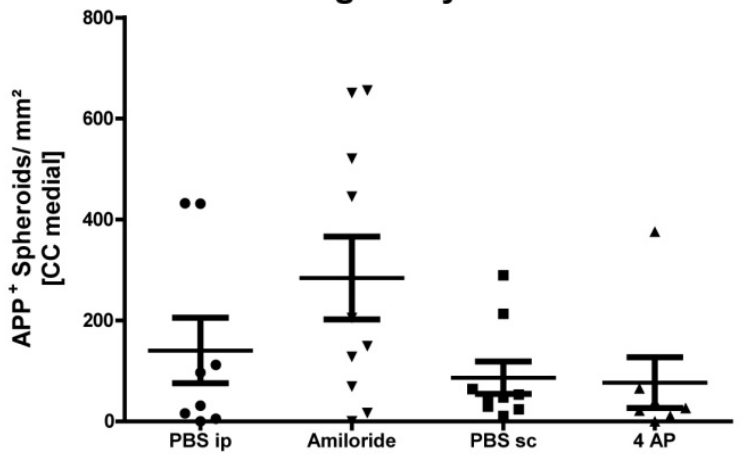

Treatment during cuprizone induced demyelination
B Influence of ion channel inhibition on acute axonal damage in the lateral CC during demyelination

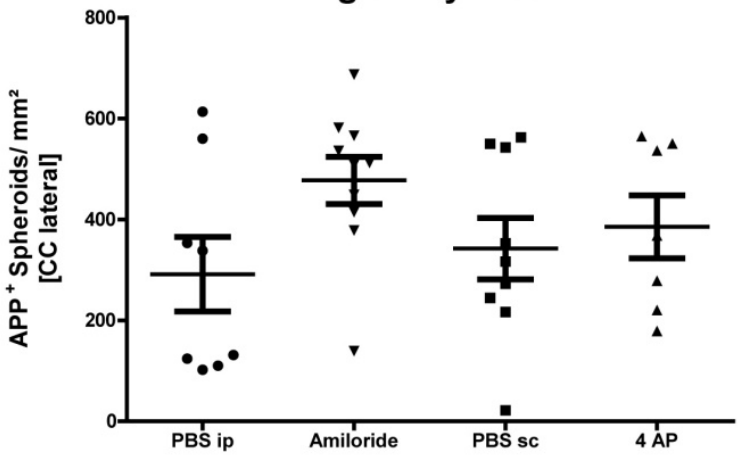

Treatment during cuprizone induced demyelination

Figure 24: Acute axonal damage of 4-aminopyridine-treated and amiloridetreated mice was not significantly different compared to vehicle-treated mice during demyelination.

The numbers of $\mathrm{APP}^{+}$spheroids in medial (A) and lateral (B) corpus callosum of mice treated with inhibitors were not significantly different to the vehicle-treated groups (mean \pm SEM; $n=7-10)$.

A

Influence of ion channel inhibition on OPCs and mature oligodendrocytes in the medial CC during demyelination

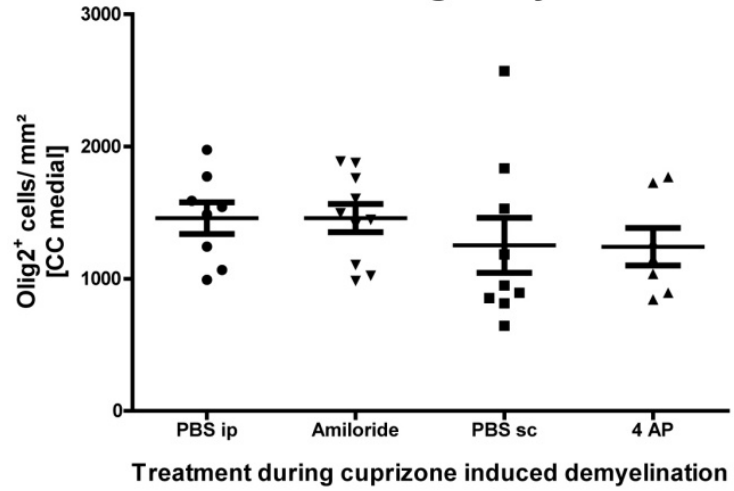

Influence of ion channel inhibition on OPCs and mature oligodendrocytes in the lateral CC during demyelination

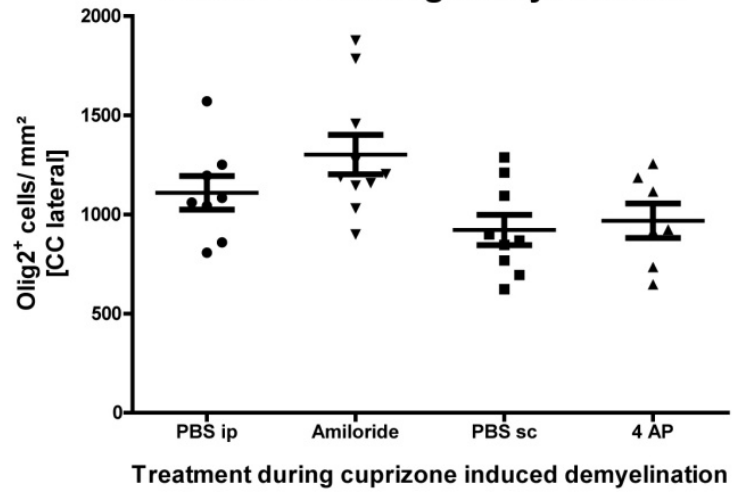

Figure 25: The numbers of oligodendrocytes were not significantly altered in inhibitor-treated mice compared to vehicle-treated mice during demyelination.

The number of Olig2 ${ }^{+}$oligodendrocytes remained unaltered in medial (A) and lateral (B) corpus callosum of mice treated with 4-aminopyridine and mice treated with amiloride in comparison to controls (mean \pm SEM; $n=7-10$ ). 
A Effect of ion channel inhibition on cuprizone-induced demyelination in the medial CC

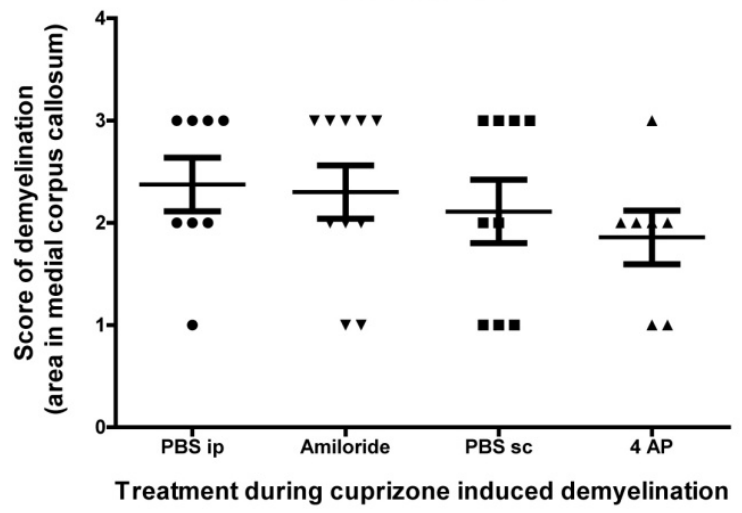

B

Effect of ion channel inhibition on cuprizone-induced demyelination in the lateral CC

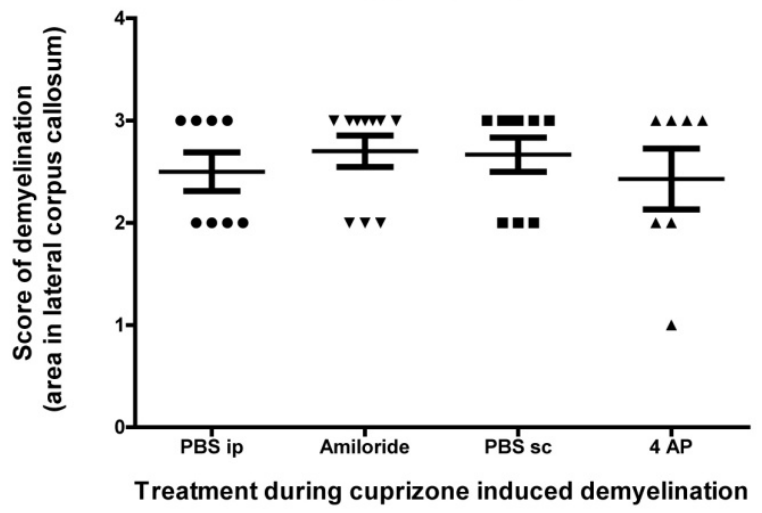

Figure 26: Mice treated with 4-aminopyridine or amiloride showed no difference in demyelination in comparison to vehicle-treated mice.

LFB-PAS staining of medial (A) and lateral (B) corpus callosum revealed a similar demyelinated area in the corpus callosum of 4-aminopyridine-treated and amiloridetreated animals, when compared to control. (mean \pm SEM; $n=7-10$ ). 


\section{$4 \quad$ DISCUSSION}

Multiple sclerosis lesions are characterized by focal inflammation, demyelination and axonal damage. Demyelination, which could occur due to CNS inflammation or injury of oligodendrocytes, leads to disturbed impulse conduction in the demyelinated axons and to modification of protein composition such as ion channels in the axonal membrane. The re-expression and redistribution of ion channels may restore the conduction across the demyelinated axonal segments. This modification of the nodal and paranodal protein clusters is partially reconstituted and comparable to normal myelinated axons by remyelination, which is considered as a mechanism of CNS repair and axonal protection (Irvine and Blakemore, 2006; Irvine and Blakemore, 2008;Lubetzki and Stankoff, 2014;Smith, 2006).

However K. J. Smith raises the question that remyelination may transiently render axons vulnerable to degeneration before long-term protection is achieved (Smith, 2006). Several findings in the past, which assessed a possible mechanism of axonal damage, evoke this question. It was shown that NO is a prominent component in inflammatory MS lesions (Smith and Lassmann, 2002) and a potent inhibitor of mitochondrial metabolism and ATP production (Brown and Borutaite, 2002). Axons consume ATP to reconstitute the intraaxonal ion concentrations after depolarization of the axonal membrane (Smith, 2006). Especially the $\mathrm{Na}^{+} / \mathrm{K}^{+}$ATPase require ATP to remove the sodium ions that enter the axon during impulse activity. When the energy demand of an axon exceeds the energy supply due to inhibited mitochondrial metabolism and ATP production, the function of $\mathrm{Na}^{+} / \mathrm{K}^{+}$ATPase will be inadequate and axons will become loaded with sodium ions. As a consequence the $\mathrm{Na}^{+} / \mathrm{Ca}^{2+}$ exchanger, which is situated in the axonal membrane, operates in reverse, so that calcium ions are imported into the axon. The increased intraaxonal concentration of intraaxonal calcium ions can cause axonal degeneration by activation of degrading enzymes (Smith, 2006). This mechanism of axonal degeneration was approved by in vitro studies, which showed axonal degeneration by NO exposure (Smith et al., 2001) and axonal protection by NO exposure and sodium channel inhibition (Kapoor et al., 2003). Furthermore, reports of sodium channel inhibition in the inflammatory demyelinating animal model EAE demonstrated an axon-protective capacity of sodium channel blocking agents (Bechtold et al., 2004;Bechtold et al., 2005;Bechtold et al., 2006; Lo et al., 2003). However for long-term protection of axons the structural repair of demyelinated axons by remyelination appeared to be necessary (Smith, 2006). Remyelination can restore nodes of Ranvier, which appear to have a normal pattern of molecular constituents, and secure conduction. In addition to the protective function of remyelination, early remyelination may render 
axons vulnerable to degeneration, before the mature nodal configuration is achieved (Smith, 2006). Smith speculated that during remyelination the sodium channels, that were distributed along the demyelinated axolemma, may be 'swept' ahead of the advancing glial plasmalemma as oligodendrocytes encircle the demyelinated axons and then extend to establish their new territories along the axons (Smith, 2006). Therefore at the edges of two adjacent oligodendrocytes that remyelinate the same axon, two focal aggregations of sodium channels may approach each other and build regions of particularly high sodium channel concentration, which may cause rapid accumulation of intraaxonal sodium ions (Smith, 2006). The proposed mechanism may be also relevant for other ion channels such as voltage-gated potassium channels, which are similarly redistributed by demyelination as sodium channels (Lubetzki and Stankoff, 2014). Especially the exposure of the voltage-gated potassium channels that are normally hidden behind the myelin sheath in the axonal membrane may result in dysregulated potassium ion homeostasis. Although the pattern of these channels is reconstituted in the axonal membrane by remyelination, an inhibition of these channels may have similar beneficial effects on demyelinated axons as the inhibition of sodium channels. Furthermore the acidic milieu caused by tissue inflammation activates acid sensing ion channels (ASICS) in the axonal membrane. ASICs can flux $\mathrm{Na}^{+}$and $\mathrm{Ca}^{2+}$ ions and thereby may further increase intraaxonal concentration of these ions, which then contribute to axonal degeneration (Friese et al., 2007; Schattling et al., 2014; Vergo et al., 2011).

Hence, one aim of my thesis was to explore the relation of early remyelination and axonal damage in MS and an animal model of MS, while a second aim was to assess the axon-protective capacity of two ion channel inhibitors, i.e. the potassium channel inhibitor 4-aminopyridine and the ASIC inhibitor amiloride, in an animal model of MS.

\subsection{Acute axonal damage in relation to early remyelination}

\subsubsection{Efficient regeneration of the corpus callosum after cuprizone diet cessation}

Demyelination of the corpus callosum was induced by cuprizone treatment for six weeks. After cuprizone withdrawal, the demyelinated area was rapidly remyelinated within a few days and almost absent after three weeks of remyelination. A sequential expression of myelin proteins was observed with CNPase as the first re-expressed myelin protein, followed by MBP and MOG, and PLP as the last re-expressed myelin protein. 


\subsubsection{The cuprizone mouse model to study remyelination}

The cuprizone mouse model is a well characterized model to study demyelination and remyelination in mice. However, the obtained demyelination and the remyelination are dependent on the dosage of cuprizone, the mouse strain, the gender and age of mice as well as the brain region analyzed and the treatment period (Hiremath et al., 1998;Jurevics et al., 2002; Lindner et al., 2008;Taylor et al., 2009;Taylor et al., 2010). For example, Lindner and colleagues used $0.2-0.3 \%$ cuprizone diet for 6 weeks and described a sequential myelin protein expression during remyelination after $0.3 \%$ cuprizone diet. The earliest time point they examined was four days after cuprizone withdrawal. By evaluating myelin protein expression by immunohistochemistry they observed a rapid increase in myelin protein expression after four days of remyelination. Although they analyzed sections of a different coronal area of the corpus callosum as it was investigated in my work, the LFB staining and myelin protein immunohistochemistry revealed a comparable pattern of sequential remyelination. Therefore my results are in line with the literature and proved the consistency of the cuprizone mouse model for studies on remyelination.

\subsubsection{Oligodendrocytes in the cuprizone mouse model}

Remyelination is dependent on the presence of oligodendrocytes. In the cuprizone model many oligodendrocyte precursor cells were present after six weeks of cuprizone treatment. However the number of mature oligodendrocytes was diminished after cuprizone challenge. During the first three days of remyelination I observed an increase in the number of oligodendrocyte lineage cells $\left(\mathrm{Olig} 2^{+}\right)$. After three days $\mathrm{Olig}^{+}$ oligodendrocytes remained relatively constant, whereas the number of $\mathrm{NogoA}^{+}$mature oligodendrocytes increased. This finding implies that oligodendrocyte proliferation is shifted to oligodendrocyte differentiation after three days of remyelination in the cuprizone model. Thus oligodendrocyte proliferation and differentiation seemed to be accomplished after one week of recovery, which is in line with the evidence of efficient remyelination.

It was reported that oligodendrocytes exhibit signs of apoptosis after two days of cuprizone intoxication and their number starts to diminish after a few days of cuprizone ingestion (Buschmann et al., 2012; Hesse et al., 2010;Matsushima and Morell, 2001). However, Mason and colleagues could demonstrate that the number of oligodendrocytes increases and that remyelination occurs during a continued exposure to cuprizone (Mason et al., 2000a;Mason et al., 2001a). Thus the presence of few NogoA ${ }^{+}$ oligodendrocytes and myelin sheaths in the LFB staining after six weeks of cuprizone 
treatment suggest that the regeneration and thereby the remyelination of the corpus callosum started during cuprizone treatment, which is in line with the literature.

\subsubsection{Acute axonal damage in the cuprizone mouse model}

\subsubsection{Acute axonal damage detectable in the corpus callosum after six weeks of cuprizone treatment}

After cuprizone challenge the corpus callosum of mice exhibited acutely damaged axons, which were presented as $\mathrm{APP}^{+}$axonal spheroids. APP accumulates at sites of axonal injury, because of a collapse or reduction of the axonal transport caused by axonal injury or damage (Kamal et al., 2000;Koo et al., 1990).

Axonal transport is very important to supply axons and their synapses with proteins, lipids and mitochondria and to remove recycled or misfolded proteins for prevention of the build-up of toxic aggregates (Millecamps and Julien, 2013). Varies cargoes are transported along microtubules either in anterograde (i.e. towards the axon synapse) or retrograde direction (i.e. towards the neuronal cell body). The transport consists of movements in a saltatory fashion: transported cargoes exhibit periods of rapid movements, pauses and directional switches. APP undergoes anterograde axonal transport and the immunoreactivity for APP identifies axonal transport disturbances, which are an indication for axonal damage and may or may not be reversible (Dziedzic et al., 2010; Trapp et al., 1998).

Remarkably, the temporal sequence of axonal swelling and destruction as a consequence of axonal damage and the reversibility of axonal damage were recently demonstrated in EAE by in vivo imaging (Nikic et al., 2011). EAE was induced in transgenic mice (Thy1-GFP-S and Thy1-CFP-S), which had a subset of medium-to-large caliber axons fluorescently labeled. The recorded axons showed a specific sequence of focal axonal degeneration: 1) prior to disruption, axonal swellings occurred along the axon at one or more discrete sites, 2) the initial disruption of an axon often occurred at putative nodes of Ranvier. The axonal disruption was shown to start synchronously to both sites. Terminal bulbs were formed on the proximal and distal axon stumps, when the fragmentation halted. Some of the recorded swollen axons recovered spontaneously. Microglia/ macrophages were determined as the initiators of axonal damage by producing ROS (reactive oxygen species) and RNS (reactive nitrogen species). Nikic and coworkers further demonstrated that the oxidative damage of mitochondria, which caused a dysfunction of these organelles, occurred simultaneously with axonal damage (Nikic et al., 2011). In addition they showed axonal damage of myelinated axons, which suggest that demyelination might not be a necessary prerequisite for axonal damage in EAE. An 
elevated mitochondrial content in demyelinated axons was described for the focal lysolecithin-induced demyelinating/ remyelinating animal model (Zambonin et al., 2011), but the pathological mechanisms in the cuprizone model are different from the autoimmune EAE based on activated encephalitic $T$ cells. In contrast to EAE the first pathological evidence in the cuprizone model is oligodendroglial apoptosis, which is followed by demyelination, microgliosis, astrogliosis and axonal damage (Buschmann et al., 2012; Lindner et al., 2009). Because of the different pathological hallmarks, the mechanisms of axonal pathology could be different in the cuprizone model. However histopathological evaluation by APP immunohistochemistry exhibited also swollen or transected axons in this model.

The axonal damage observed in the cuprizone-treated mice could be either a result of a direct toxic effect on the axons or a secondary effect of tissue injury such as oligodendrogliopathy, demyelination and microglial activation. It is reported for the cuprizone mouse model that axonal damage and degeneration occur (Mason et al., 2001a; Stidworthy et al., 2003), although cuprizone seemed to have no direct toxic effect on axons (Irvine and Blakemore, 2006). Irvine and Blakemore compared the number of damaged axons (SMI-32 ${ }^{+}$, non-phosphorylated neurofilament) in the corpus callosum of Swiss and C57BL/6 mice, which were treated with high or low doses of cuprizone (Irvine and Blakemore, 2006). The Swiss mice were treated with approximately two times higher dose of cuprizone, but exhibited less axonal spheroids than the C57BL/6 mice. In the same study they compared the axonal damage in young and old C57BL/6 mice. Old mice showed increased axonal damage compared to young mice, but the old Swiss mice treated with high dose of cuprizone exhibited less axonal spheroids compared to old C57BL/6 mice. The authors concluded that the dosage of cuprizone was most likely not directly toxic for axons (Irvine and Blakemore, 2006). However the study was performed with small groups of mice and in different strains. In addition to this in vivo study showed cuprizone no direct toxic effect on neuronal viability in vitro (Benardais et al., 2013). In this study a neuronal cell line was used, which exhibit no formation of axons. Thus, it has not been absolutely clarified whether cuprizone has a direct toxic effect on axons.

\subsubsection{Acute axonal damage decreased during remyelination}

The number of the axonal spheroids observed in the present work decreased continuously during remyelination. Remyelination seemed to be neuroprotective, while the cuprizone-induced demyelination appeared to be harmful to axons (Irvine and Blakemore, 2006;Irvine and Blakemore, 2008). In 2008 Irvine and Blakemore studied the potential of remyelination to protect axons. The oligodendrocyte progenitor cells were depleted by $\mathrm{X}$-irradiation in the cuprizone model, which resulted in remyelination failure. 
X-irradiated, cuprizone-treated mice exhibited more axonal damage (SMI- $32^{+}$, nonphosphorylated neurofilament) and axonal loss in comparison to non-irradiated, cuprizone-treated mice after cuprizone withdrawal. The remyelinating capacity of the $X-$ irradiated brain was restored by transplantation of $\mathrm{GFP}^{+}$embryonic neurospheres, which differentiate mainly into oligodendrocytes. The transplantation of the embryonic neurospheres into the brain of $X$-irradiated, cuprizone-treated mice resulted in increased axonal survival and decreased axonal damage. These results suggested an axonprotective role of remyelination in the cuprizone mouse model.

Most of the investigators, who explored mechanisms of demyelination, remyelination and axonal damage in the cuprizone mouse model, used time intervals of weeks or months (Gudi et al., 2009; Hiremath et al., 1998;Lindner et al., 2008;Lindner et al., 2009;Manrique-Hoyos et al., 2012;Skripuletz et al., 2013;Stidworthy et al., 2003). In this study the time intervals of days enabled a detailed analysis of the early remyelination and axonal damage in mice. Acute axonal damage decreased continuously during recovery presumably due to rapid and efficient oligodendrocyte differentiation and remyelination. However, acute axonal damage ( $9 \mathrm{APP}^{+}$spheroids/ $\left.\mathrm{mm}^{2}\right)$ was shown to still occur after long term remyelination (28 weeks) (Manrique-Hoyos et al., 2012). The axonal damage, which was evaluated after one (163.3 $\mathrm{APP}^{+}$spheroids/ $\mathrm{mm}^{2}$ ) or three (129.2 $\mathrm{APP}^{+}$spheroids/ $\mathrm{mm}^{2}$ ) weeks of remyelination in the present work, was much higher than the axonal damage observed in the study of Manrique-Hoyos and colleagues. Therefore, the majority of the axonal damage probably occurred during cuprizone treatment and was still detectable during remyelination.

Damaged or transected axons could be detected by immunohistochemistry for APP a few hours after injury (McKenzie et al., 1996;Otsuka et al., 1991), but could be also observed two to four weeks after the injuring insult (Bramlett et al., 1997; Pierce et al., 1996). APP as a marker was established for various disorders of the CNS including multiple sclerosis (Bitsch et al., 2000;Kornek et al., 2000;Kuhlmann et al., 2002). Hence the measured acute axonal damage is likely a result of the demyelination induced by cuprizone treatment and continues to be detected during remyelination.

\subsubsection{Decreased activated microglia and sustained reactive astrocytes during remyelination in the cuprizone mouse model}

The corpus callosum of mice treated for six weeks with cuprizone showed many activated microglia and reactive astrocytes. The number of reactive astrocytes remained relatively constant during remyelination in contrast to the expeditious decrease of activated microglial cells. Furthermore a localization of cell processes of reactive astrocytes with acutely damaged axons was observed during remyelination. 
Several studies reported activation of microglia and increased numbers of reactive astrocytes induced by cuprizone ingestion (Gudi et al., 2009; Hiremath et al., 1998; Lindner et al., 2009;Mason et al., 2004). However, the number of activated microglia decreased after cuprizone diet cessation (Lindner et al., 2009;Mason et al., 2004; Matsushima and Morell, 2001;Remington et al., 2007), while astrogliosis remained unaltered during recovery (Hibbits et al., 2012;Skripuletz et al., 2011).

\subsubsection{Activated microglia during remyelination}

Microglia are the resident macrophages of the CNS and can migrate throughout the parenchyma upon activation (Benarroch, 2013;Hanisch and Kettenmann, 2007; Kettenmann et al., 2011;Kettenmann et al., 2013). In a healthy environment the microglial cell appears ramified and is known as the "resting" microglia. The resting microglia show a small soma with fine cellular processes. These cells are evenly distributed and each cell seems to occupy a defined territory. However, they are not quiescent, but rather constantly scanning their environment with their motile processes to survey the tissue (Kettenmann et al., 2013). Once a threat to the CNS has been detected, they change their morphology from ramified to amoeboid, which is also considered as activated microglia. These amoeboid microglial cells can migrate towards the site of pathological alteration, proliferate and phagocytose. This "activation" of microglial cells is rather a change in activities than activation per se, because of the beneficial role of resting microglia in CNS remodeling and repair as well as synaptic plasticity. In fact, dependent on the environmental factors (e.g. inflammation, tissue damage) activated microglia exhibit different functions, which are not necessarily detrimental (Benarroch, 2013; Hanisch and Kettenmann, 2007; Kettenmann et al., 2011;Kettenmann et al., 2013). In the NAWM of MS patients, for example, isolated microglia appeared to be activated, but did not respond to inflammatory stimuli (Melief et al., 2013). Microglia were isolated from post-mortem brain and their appearance evaluated by FACS analysis. The microglial cells exhibited increased size, granularity and CD45 expression, but unresponsiveness to LPS in vitro (Melief et al., 2013). Furthermore the microglial activation goes not only in one direction: activated microglia and peripheral macrophages can integrate into the CNS parenchyma and acquire a microglia phenotype (Benarroch, 2013; Hanisch and Kettenmann, 2007;Kettenmann et al., 2011;Kettenmann et al., 2013).

The role of microglial cells in the cuprizone model was investigated by Remington and colleagues in 2007 (Remington et al., 2007). Cells were isolated from the corpus callosum of cuprizone-treated mice during demyelination and after remyelination and were evaluated by FACS analysis. The microglial cells and macrophages were both CD11b positive, but could be distinguished by CD45 expression. Microglia exhibited low 
expression of CD45, whereas macrophages showed high expression of CD45. During cuprizone-induced demyelination the microglia became activated and migrated to the corpus callosum, in which they further proliferated. In the fifth week of cuprizone challenge the microglial/ macrophage response was maximal. But the macrophages constituted only $0.5 \%$ of the CD11b positive cells. Therefore the macrophage response in the cuprizone model resembled rather the macrophage response seen after axonal injury than the massive macrophage infiltration seen in MOG-EAE (Remington et al., 2007). Furthermore at the time of maximum response approximately $\sim 30 \%$ of the responding microglia were immigrants from the periphery (Remington et al., 2007), although blood brain barrier remains intact in the cuprizone model (Bakker and Ludwin, 1987; Kondo et al., 1987;McMahon et al., 2002). Remington and colleagues used bone marrow chimeras, which were whole-body irradiated previous to bone marrow transplantation, to investigate the engraftment of peripheral monocytes into the CNS. The engraftment of peripheral monocytes exhibiting a microglial phenotype was reported to occur only due to preconditioning of the brain such as irradiation (Mildner et al., 2007). Thus, the increased numbers of activated microglia in the demyelinating mouse brain originated from local proliferation of microglia. In another approach microglia exhibited mainly a phagocytic activity during demyelination and remyelination, which was accompanied by an upregulation of IGF1 and FGF2 (Voss et al., 2012). Especially the upregulation of the growth factors and the phagocytic activity suggested a regenerative role of microglia in the cuprizone model. In addition to these comprehensive studies of microglia, the number of activated microglia was histopathologically determined in the cuprizone model using either Mac3 or RCA-1 as a marker (Gudi et al., 2009; Hiremath et al., 1998; Lindner et al., 2009; Mason et al., 2004; Voss et al., 2012). The maximum of the density of activated microglial cells emerged between four to six weeks of cuprizone treatment in these studies. The different maximum of the numbers of activated microglial cells might be caused by the different regions analyzed or the different markers that were used. However in the studies, which included analysis of remyelination, a prominent decrease of activated microglial cells was observed either during the end of cuprizone treatment or during remyelination (Gudi et al., 2009;Lindner et al., 2009;Mason et al., 2004; Remington et al., 2007; Voss et al., 2012). It was reported that activated microglial cells coincided with oligodendrocyte apoptosis (Mason et al., 2004) and axonal damage in the cuprizone model (Lindner et al., 2009). However several studies demonstrated a beneficial role of activated microglia, reactive astrocytes and their released cytokines on oligodendrocyte differentiation and remyelination in the cuprizone model (Arnett et al., 2001;Diemel et al., 2003;Hinks and Franklin, 1999;Mason et al., 2001b;McKinnon et al., 
1993; Patel et al., 2010). It was shown that cytokines such as IL1 $\beta$, TNFa, CXCL12 and TGF $\beta$ promote oligodendrocyte differentiation and remyelination in the cuprizone model. Therefore, the microglia detected during remyelination, which appeared to be activated, are most likely rather promoting CNS repair than CNS damage. Moreover the prominent reduction in the number of activated microglial cells during remyelination suggests CNS regeneration.

\subsubsection{Reactive astrocytes during remyelination}

Astrocytes tile the entire CNS and have important functions within the CNS: involvement in synaptic transmission and energy metabolism, regulation of neurogenesis, maintenance of the blood-brain-barrier, and control of blood flow (Gudi et al., 2014; Sofroniew and Vinters, 2010). Reactive astrogliosis occurs due to CNS injury and disease, which is accompanied by various cellular changes including upregulation of GFAP (Sofroniew, 2009; Sofroniew and Vinters, 2010). It is not a single uniform process or an all-or-none response. The process of reactive astrogliosis is finely and continuously graded progressive changes in gene expression and cellular changes. The extent of astrogliosis depends on the severity of the triggering insult. Mild and moderate forms of reactive astrogliosis exhibit the potential of resolution with normal-appearing astrocytes afterwards, when the triggering insult disappeared. In contrast severe forms of reactive astrogliosis in response to overt tissue damage and inflammation build a glial scar with overlapping astrocytic cell processes that incorporate newly proliferated cells (Sofroniew, 2009; Sofroniew and Vinters, 2010).

The role of reactive astrocytes in demyelinating diseases is controversially discussed (Brück et al., 2012; Colombo et al., 2012;Gudi et al., 2011; Lee et al., 2012; Patel et al., 2010;Skripuletz et al., 2013;Stadelmann et al., 2002). Reactive astrocytes were shown to have detrimental effects in EAE and induce neurodegeneration in vitro (Colombo et al., 2012). However several reports demonstrated the beneficial impact of astrocytes in the cuprizone mouse model with regard to remyelination (Gudi et al., 2011;Patel et al., 2010;Skripuletz et al., 2013). The number of reactive astrocytes was shown to increase after three weeks of cuprizone-induced demyelination (Gudi et al., 2009; Hiremath et al., 1998) and to persist during remyelination (Hibbits et al., 2012;Skripuletz et al., 2011). However, depletion of astrocytes in the cuprizone mouse model resulted in decreased microglial activation and impaired microglial recruitment, which caused a delay in clearance of myelin debris (Skripuletz et al., 2013). Although the demyelination seemed to be prevented, the remaining myelin appeared loose and frayed. The depletion of astrocytes did not impede oligodendrocyte apoptosis and axonal damage. Furthermore the proliferation of oligodendrocyte progenitors and the 
remyelination were decelerated, while diminished numbers of oligodendrocytes were observed (Skripuletz et al., 2013). In addition, the expression of various cytokines and growth factors was described to be upregulated in reactive astrocytes during remyelination (Gudi et al., 2011;Patel et al., 2010;Skripuletz et al., 2013). Some of these factors such as CXCL10, CXCL12, TGF $\beta$, TNFa, IGF1 and CNTF suggest a beneficial role of reactive astrocytes during remyelination. These factors were shown to promote the recruitment of microglia or oligodendrocyte differentiation. Thereby clearance of myelin and remyelination were facilitated in the cuprizone mouse model (Gudi et al., 2011; Patel et al., 2010;Skripuletz et al., 2013). The persisting reactive astrogliosis observed during remyelination in the current study was moderate to severe, but without any scar formation and in line with the literature (Hibbits et al., 2012;Skripuletz et al., 2011). Furthermore the efficient remyelinating process demonstrated in this work together with the evidence from the recent literature (Gudi et al., 2011;Patel et al., 2010; Skripuletz et al., 2013) suggests a beneficial function of reactive astrocytes in remyelination. Thus astrocytes seem to promote CNS repair. In addition to their role in remyelination it was shown that astrocytic processes envelop synapses and are in close contact to axons at the nodes of Ranvier (Sofroniew and Vinters, 2010). The cell processes of reactive astrocytes might be attracted by acutely damaged axons and suggests that the astrocytes might have an impact on axonal damage, which has to be further clarified in future studies.

\subsubsection{Expression of TGF $\beta$ and TNF $\alpha$ was upregulated during demyelination and remyelination}

The expression of TGF $\beta$ and TNFa was upregulated during demyelination and remyelination. In contrast to TNFa, the expression of TGF $\beta$ decreased after one week of remyelination. Furthermore the expression level of IL6 might be reduced in the cuprizone mouse model. These results are in line with the literature (Arnett et al., 2001;Biancotti et al., 2008; Gudi et al., 2011; Krauthausen et al., 2014).

Expression levels of many cytokines and chemokines, e.g. IL-1 $\beta$, TGF $\beta$, TNFa, IL6, CXCL10, CCL2 and CCL3, were shown to be regulated in the cuprizone mouse model (Arnett et al., 2001;Biancotti et al., 2008;Buschmann et al., 2012;Gudi et al., 2011;Krauthausen et al., 2014;Mason et al., 2000b;Mason et al., 2001b;Voss et al., 2012). Many cytokines are multipotent factors that can be beneficial for some cells and detrimental for others. The inflammatory cytokine TNFa, for example, can induce apoptosis and also promote cell proliferation dependent on the target cell and its receptors (Caminero et al., 2011;Mc et al., 2011). Several scientists showed alleviation of EAE by treatment with anti-TNFa antibodies (Korner et al., 1997; Ruddle et al., 
1990; Selmaj et al., 1991; Suvannavejh et al., 2000), but in clinical trials using antibodies against TNFa MS patients showed an exacerbation of the disease (1999; van Oosten et al., 1996). In line with the clinical trials Arnett and colleagues demonstrated that TNFa promotes oligodendrocyte progenitor proliferation and remyelination in the cuprizone mouse model (Arnett et al., 2001). A promoting impact on oligodendrocyte proliferation and remyelination was also reported for TGF $\beta$ (Diemel et al., 2003; Hinks and Franklin, 1999;McKinnon et al., 1993). For this reason the increased expression levels of TGF $\beta$ and TNFa measured in the cuprizone model might implement regenerative rather than degenerative processes during remyelination.

\subsubsection{The subpopulation of myelinated damaged axons increased during remyelination}

A considerable number of myelinated axonal spheroids were observed after cuprizone treatment. In addition, a significant increase of myelinated spheroids could be determined after one week of remyelination.

Axonal spheroids, which were ensheathed with myelin, were observed after long term remyelination in the cuprizone mouse model (Manrique-Hoyos et al., 2012). Manrique-Hoyos and coworkers further showed myelinated axonal spheroids in chronic remyelinated MS lesions. In line with their findings the immunhistochemical study of axonal spheroids revealed that $25 \%$ of the spheroids appeared myelinated after successful remyelination. The temporal relation of axonal spheroids and early remyelination suggests that axons could be remyelinated independent of any transport disturbance. This hypothesis is further supported by the work on chronically demyelinated axons (Foote and Blakemore, 2005a;Mason et al., 2004). It was demonstrated that chronic demyelinated axons can still be remyelinated, which appeared to be rather dependent on oligodendrocytes and environmental factors than on the chronically demyelinated axons themselves. Moreover a functional axonal transport does not seem to be a necessary precondition for the proper establishment of an impeccable myelin sheath.

\subsubsection{Contiguous large demyelinated areas without intact myelin sheaths in the corpus callosum after cuprizone challenge}

The electron microscopic analysis of the corpus callosum of cuprizone-treated mice revealed large demyelinated areas with a few altered, but still myelinated axons, which appeared to contain increased numbers of mitochondria. These findings are in line with the literature (Crawford et al., 2009;Dikranian et al., 2008;Stidworthy et al., 2003) and possibly reflect axons with transport disturbance. Remyelinated axons were present 
after six weeks of cuprizone treatment, which suggests remyelination during cuprizone treatment and is in line with the literature (Mason et al., 2000a;Mason et al., 2001a).

\subsection{7 (Re)myelinated damaged axons were also present in early remyelinating early active MS lesions}

Myelinated damaged axons were noticed by Trapp and colleagues in active MS lesions (Trapp et al., 1998), but whether myelinated damaged axons could be found in early remyelinating, early active MS lesions was unknown. Here in all four investigated cases myelinated axonal spheroids were observed. The spheroids were located within a contiguous demyelinating area with ongoing early remyelination. This observation suggests that remyelination in MS might occur similarly to the mechanism described for the cuprizone model. But this hypothesis has to be proved by further investigation of early active demyelinating lesions with no sign for remyelination versus early active demyelinating lesions with ongoing early remyelination. Another intriguing issue is that the cuprizone model is a toxic model not involving important $\mathrm{T}$ lymphocyte infiltration, primarily to investigate mechanisms of remyelination. Some mechanisms of remyelination in MS might resemble those identified in the cuprizone model. However the focal inflammation in MS could influence the process of remyelination and the occurrence of axonal damage. Inflammation, for example, was shown to support remyelination in another animal model (Foote and Blakemore, 2005b). Hence, more MS lesions have to be analyzed to determine the relevance of the proposed mechanism of remyelination of axons showing a disturbance of axonal transport in MS.

\subsubsection{Remyelinated MS lesions exhibited less acute damaged axons than chronic inactive MS lesions}

Remyelination is considered as a regenerative process, which is capable of protecting axons after demyelinating events (Irvine and Blakemore, 2008;Kornek et al., 2000; Kuhlmann et al., 2002; Murray et al., 2001). The axon-protective capability of remyelination was demonstrated in these studies by investigation of MS related animal models and chronic MS lesions. Restoration of neurological function or reduction of axonal damage could be an indication of axon protection. Murray and colleagues, for example, reported in a viral animal model of MS a partial restoration of neurological functions coinciding with spontaneous remyelination (Murray et al., 2001). In addition to that, a decrease of axonal damage due to remyelination was shown in the cuprizone mouse model by Irvine and Blakemore (Irvine and Blakemore, 2008). Furthermore remyelination is beneficial for axonal health in MS lesions in the long-term as shown by Kornek et al. and Kuhlmann et al. (Kornek et al., 2000;Kuhlmann et al., 2002). Here, the 
evaluated remyelinated MS lesions exhibited less acutely damaged axons than chronic inactive lesions, which is in line with the literature (Kornek et al., 2000;Kuhlmann et al., 2002). This finding therefore proves the axon-protective capability of remyelination in multiple sclerosis.

\subsubsection{Colocalization of the paranodal protein Caspr with APP $^{+}$spheroids in the cuprizone mouse model}

The current study showed an accumulation of Caspr, a paranodal protein, at sites of acute axonal injury, which was reported previously for sodium channels, calcium channels and sodium calcium exchanger in MS and EAE (Craner et al., 2004a;Craner et al., 2004b;Herrero-Herranz et al., 2008; Kornek et al., 2001). The immunohistochemistry for Caspr revealed diffuse and vesicle-like distribution of Caspr in axonal spheroids.

Paranodal (e.g Caspr) and nodal proteins (e.g. $\mathrm{Na}_{\vee}$ channels) are arranged in multiprotein complexes, which are clustered into distinct domains at the nodes of Ranvier by myelin formation. The assembly of the paranodal complex is initiated by the interaction of neurofascin 155 in glia to a complex of contactin and contactin associated protein (Caspr) in axons. The complex is then stabilized by a specialized axonal cytoskeleton, which consists of several proteins such as ankyrin $B$ and $\alpha / \beta I I$ spectrin (Sherman and Brophy, 2005;Simons and Trajkovic, 2006). It was shown by Eisenbach et.al. that Caspr expression and localization occur in a specified sequence (Eisenbach et al., 2009). The sequence, which was shown in a myelinating cell culture model, consisted of four steps during myelination: First, Caspr was weakly and uniformly expressed in the absence of oligodendrocytes; second, clustering of Caspr at sites of initial contact of oligodendrocytes and axons; third, membrane expression of Caspr was increased in axonal segments ensheathed with myelin; and fourth, Caspr was excluded from internodes and accumulated at paranodes as myelination proceeded. These processes could be applied for myelination in development, because of the embryonal and neonatal cell culture system. Therefore, these mechanisms can not necessarily be applied to the demyelinating and remyelinating situation. However, Zoupi and colleagues illustrated the alterations of paranodal and nodal protein distribution in EAE and the cuprizone model (Zoupi et al., 2013). Cuprizone-induced demyelination strongly affected the paranodal protein clustering, which was accompanied by a reduced nodal density and the appearance of elongated nodes. In addition immunoreactivity for Caspr was reduced, diffused or completely absent. However the protein complexes reappeared after remyelination in the cuprizone model. Furthermore a study in 2003 revealed a similar diffused or reduced expression pattern of Caspr in chronic MS lesions (Wolswijk and Balesar, 2003). These data together with the observation of colocalization of Caspr and 
ion channels at sites of acute axonal injury suggest two possible hypotheses: 1) axons might be more vulnerable due to new myelin formation, 2) the deposition of Caspr at sites of axonal injury might be a consequence of disturbed axonal transport. The findings of the present study support the second suggestion, because of the observed vesicle-like immunoreactivity for Caspr, which was not restricted to the axonal surface. Therefore intraaxonal accumulation of Caspr might be a marker for axonal damage, but this issue has to be investigated in more detail in future studies.

\subsubsection{Conclusion}

In the first part of my thesis I focused initially on the CNS pathology in the cuprizone model, in particular in the corpus callosum of mice after cuprizone treatment and after cuprizone diet cessation, to investigate the relation of remyelination and axonal damage. The pathology of the cuprizone-treated mice revealed regenerative processes such as remyelination after cuprizone withdrawal. The remyelination of the CNS appeared as follows: few mature oligodendrocytes were present after six weeks of cuprizone challenge and the oligodendrocyte proliferation and differentiation were accomplished after one week of recovery accompanied by an efficient remyelination. Furthermore, the prominent decrease of activated microglia two days after cuprizone withdrawal points also to CNS regeneration and repair. The continuous reduction of acutely damaged axons during remyelination is in line with these findings. Although reactive astrocytes persists in this model the upregulated cytokines are described to be beneficial for remyelination in the cuprizone model. To unravel the relation of acute axonal damage and remyelination in more detail a comprehensive analysis by confocal microscopy was performed. The analysis by confocal microscopy revealed three subpopulation of axonal spheroids: un-/ or demyelinated, partially myelinated and myelinated spheroids. The number of myelinated axonal spheroids increased significantly after one week of remyelination in spite of a decrease of the total number of axonal spheroids and strong regenerative processes. The obtained data implicate that most of the spheroids detected during remyelination in the model are a sign of damaged axons which sustained damage during the demyelinating period before. This would imply that those damaged axons are capable of gaining a new myelin sheath - a finding with far reaching consequences. A functional axonal transport is not a necessary precondition for the proper establishment of an impeccable myelin sheath. This finding shifts the common understanding of remyelination as an axon-protective mechanism towards an active role in preserving axon integrity. Remyelination and the direct long-segment contact of the oligodendrocyte with the damaged axon might act as a kind of patch which assists the axonal regenerative process. Moreover these findings suggest that remyelination may 
occur randomly without the perfect molecular match between an oligodendrocyte and an axon.

Besides the animal model of cuprizone-induced de- and remyelination, the content of remyelination and axonal damage was also investigated in MS lesions. For this, human brain biopsy tissue samples were taken from the archived material at the Department of Neuropathology of the University Medical Center Göttingen.

Myelinated spheroids were also observed in early remyelinating, early active MS lesions, which implies probably similar mechanisms of remyelination in MS. However, the cuprizone mouse model is a mechanistic model and not disease-related. Hence, the detailed investigation in MS lesions may result in another mechanism of remyelination.

\subsection{Ion channel blockage during demyelinating and remyelinating processes in the CNS}

\subsubsection{Axonal pathology during demyelination and remyelination in mice treated with ion channel inhibitors-potentially beneficial?}

APP, as a marker for acute axonal damage, colocalizes often with voltage-gated sodium channels and sodium calcium exchanger (Craner et al., 2004a;Craner et al., 2004b;Herrero-Herranz et al., 2008). Additionally, the distribution of nodal and paranodal proteins is modified by demyelination as well as remyelination (Coman et al., 2006; Craner et al., 2004b;Dupree et al., 2004). It is discussed whether the redistribution of nodal and paranodal proteins and the resulting dysregulation of the ion homeostasis makes axons more vulnerable (Black et al., 2006;Black et al., 2007;Craner et al., 2004b;Smith, 2006;Smith, 2007). Furthermore, studies demonstrated an amelioration of EAE by the ion channel inhibitors 4-aminopyridine and amiloride (Friese et al., 2007; Gobel et al., 2013; Vergo et al., 2011). However the inhibition of voltage-gated potassium channels with 4-aminopyridine and the acid sensing ion channels with amiloride revealed no protective effect on acutely damaged axons during demyelination and remyelination in the present study. Furthermore the administration of 4aminopyridine preceding remyelination did not show any protective effect on acutely damaged axons. For this reason the failure to protect from axonal transport disturbance cannot be explained.

In general two pathogenic models were proposed for MS: the 'outside-in model' and the 'inside-out model' (Witte et al., 2014). In the first model the immune cells from the periphery and activated microglia attack myelin and oligodendrocytes, which results in axonal damage as a bystander effect and, later on, as a consequence of the loss of myelin trophic support. On the other hand inflammatory processes may occur secondarily to a primary cytodegenerative process in resident CNS cells (e.g. oligodendrocytes and 
neurons/ axons) or alterations in the myelin-axon interaction. There is evidence for both scenarios in the literature. In both models a mitochondrial dysfunction is proposed to be the major reason for axonal damage and degeneration. Pathological alterations of mitochondria were detected in EAE and MS (Witte et al., 2014). ROS and RNS produced by macrophages and activated microglia were most likely one important reason for mitochondrial dysfunction during active lesion formation (Nikic et al., 2011; Witte et al., 2014). Remarkably, the detoxification of ROS reversed mitochondrial dysfunction and rescued axons from axon degeneration in EAE (Nikic et al., 2011;Witte et al., 2014). Most axons survived the inflammatory attack in progressive MS cases and became chronically demyelinated (Witte et al., 2014). Some of these axons degenerated and showed mitochondrial pathology, while over half of the demyelinated axons appeared to be intact with uninjured mitochondria. Anyhow these mitochondria exhibited enlarged size and respiratory chain enzyme activity (Mahad et al., 2009). Furthermore motile mitochondria were reported to be transported with greater speed in retrograde and anterograde direction upon demyelination in vitro (Kiryu-Seo et al., 2010). The increased mitochondrial content in axons might be adaptive or compensatory mechanism to demyelination. Once the energy demand is outreaching the capacity of energy supply by mitochondria, the increased mitochondrial content is not enough to protect an axon against damage and degeneration (Trapp and Stys, 2009). Especially the redistribution and increased expression of voltage-gated sodium channels might be a risk factor for demyelinated axons. These channels could result in an increased intracellular $\mathrm{Na}^{+}$ concentration, which in turn causes a high energy demand to remove the excess of $\mathrm{Na}^{+}$ by the $\mathrm{Na}^{+} / \mathrm{K}^{+}$ATPase from the axon. An insufficient mitochondrial energy supply would lead to the reversal of intraaxonal $\mathrm{Na}^{+}$of the $\mathrm{Na}^{+} / \mathrm{Ca}^{2+}$ exchanger by the excess of intraaxonal $\mathrm{Na}^{+}$and thereby to an increase of the intraaxonal $\mathrm{Ca}^{2+}$ concentration. The elevated $\mathrm{Ca}^{2+}$ concentration will eventually trigger deleterious events, which would result in further destabilization of the axon accompanied by ROS production and axon degeneration (Witte et al., 2014). The recent literature regarding ion channel inhibitors investigated in EAE (Friese et al., 2007;Gobel et al., 2013; Vergo et al., 2011) might indicate therefore an axon-protective potential of ion channel inhibitors for demyelinated axons. However the reports showed controversial results, i.e. it seems that some blockers have the capability to reduce the axonal pathology (Friese et al., 2007; Vergo et al., 2011), while others only improve the motility of mice with EAE (Gobel et al., 2013). The inhibitors analyzed in the present work appeared to have no impact on axonal preservation in the cuprizone mouse model. These data suggest that ion channel inhibition might not be efficient enough to prevent axonal pathology in every pathological 
context. Furthermore, the ion channel inhibition did not modify the proportion of myelinated damaged axons.

\subsubsection{Increased numbers of microglial cells during remyelination by ion channel inhibition, but unaltered reactive astrogliosis}

The number of microglial cells was increased due to ion channel inhibition, whereas the number of astrocytes remained unaltered.

Recently the expression of several ion channels on microglia and astrocytes was discovered to be upregulated in MS and EAE (Schattling et al., 2014). The function of the ion channels on astrocytes ( $\left.\mathrm{Na}_{\vee} 1.5, \mathrm{P} 2 \times 7\right)$ is speculated to be beneficial for regulating ion homeostasis, but awaits further investigation in MS and its animal models. Furthermore two ion channels ( $\mathrm{Na}_{\mathrm{v}} 1.5, \mathrm{~N}$-type $\mathrm{Ca} 2+\alpha 1 \mathrm{~B}$ subunit) were reported to be upregulated in microglia in EAE and MS. The inhibition or the genetic ablation resulted in reduced inflammatory infiltrates in EAE accompanied by reduced cytokine production, phagocytic activity and cell migration (Schattling et al., 2014). Despite this regulation of ion channels in astrocytes and microglia in MS and EAE, it is unknown whether these cells are influenced by the ion channel inhibitors 4-aminopyridine and amiloride. The increase in the number of activated microglial cells observed points to an effect of the inhibitors on these cells. In contrast, the inhibitors seemed to have no effect on astrocytes. For this reason further analysis of the effects on these cell types is necessary to verify the potential impact of these ion channel inhibitors.

\subsubsection{Demyelination and remyelination in the cuprizone mouse model- beneficial effects by ion channel inhibition}

The inhibition of voltage-gated potassium channels during remyelination revealed an increase in oligodendrocyte cell number. Furthermore, the demyelination was not significantly modified by $\mathrm{K}_{\mathrm{V}}$ channel inhibition.

It was shown by Bacia et al (Bacia et al., 2004) that 4-aminopyridine decreased the number of oligodendrocytes during remyelination. However they used a high dosage of cuprizone $(0.4 \%)$, which might cause a sophistication of the results obtained by a higher cuprizone challenge for oligodendrocytes. But in addition to that it is reported that oligodendrocytes express several voltage-gated potassium channels (Herrero-Herranz et al., 2007; Schmidt et al., 1999;Tiwari-Woodruff et al., 2006). Some channels were shown to be important for oligodendrocyte differentiation (Herrero-Herranz et al., 2007;TiwariWoodruff et al., 2006). Interestingly the ion channel $\mathrm{K}_{\mathrm{V}} 1.4$ was reported to be expressed in oligodendrocyte precursor cells and to be absent in the healthy CNS (Herrero-Herranz et al., 2007). After induction of EAE the ion channel was re-expressed in proliferating 
oligodendrocytes, which co-expressed CNPase and were actively remyelinating naked axons (Herrero-Herranz et al., 2007). Therefore the increased numbers of OPCs and mature oligodendrocytes during remyelination might demonstrate a potential of ion channel inhibitors to regulate oligodendrocyte maturation.

\subsubsection{Conclusion}

In the second part of my thesis I evaluated the potential of 4-aminopyridine, a potassium channel inhibitor, and amiloride, an acid sensing ion channel inhibitor, to reduce the axonal damage and thereby preserving the axonal integrity during demyelination and remyelination. The CNS pathology of mice, treated with 4aminopyridine or amiloride, was analyzed during cuprizone-induced demyelination and during remyelination after diet cessation. There was no evidence of reduction of the axonal damage by 4-aminopyridine administration. In contrast, the inhibition of potassium channels seemed to enhance microglial activation after remyelination, which may be more relevant in other animal models with a higher impact of inflammatory cells. Administration of 4-AP during remyelination leads to an increased number of oligodendrocytes after two weeks of recovery. Furthermore, demyelination was not modified by 4-aminopyridine administration during cuprizone treatment.

Amiloride administration showed a trend to increase microglial activation, but did not alter axonal damage or other pathological parameters.

In conclusion, these results suggest that neither 4-aminopyridine nor amiloride could change the axonal damage in the cuprizone model. However, the increased numbers of oligodendrocytes in the corpus callosum of 4-AP-treated mice after remyelination point to a protective and regenerative effect. This study provides evidence for ion channels as a potential target to promote remyelination. 


\section{$5 \quad$ SUMMARY AND CONCLUSIONS}

Axonal damage and degeneration are pervasive in the CNS of MS patients and cause persisting disabilities. The formation of new nodes of Ranvier is discussed in the literature as a potential factor that renders axons more vulnerable to axonal damage and degeneration in addition to demyelination, inflammation and oligodendroglial death. One mechanism that might contribute to the pronounced vulnerability of axons to axonal damage and degeneration during early remyelination is ion channel redistribution and reexpression in demyelinated axons which develop a new myelin sheath.

In my thesis I could show in the cuprizone mouse model that early remyelination did not render axons more vulnerable to axonal damage. Remarkably, the findings of this study implicate that remyelination of demyelinated axons may occur independently of functional axonal transport.

Furthermore, the pharmacologic inhibition of $\mathrm{K}_{\mathrm{V}}$ channels or ASICs did not modify the extent of axonal damage in the cuprizone model. Therefore the redistribution of $\mathrm{K}_{\mathrm{V}}$ channels or the activation of ASICs during remyelination may not play an important role in rendering axons more vulnerable to axonal damage.

In conclusion, my work contributes to the elucidation of the mechanisms that are important for axonal preservation and remyelination. Findings in the literature point to a distinct expression of proteins in the axonal membrane to achieve successful remyelination (Charles et al., 2002). However, the findings of my thesis demonstrate that axons with axonal transport disturbances can be remyelinated. Reports on oligodendroglial differentiation suggest that a differentiation block of oligodendrocytes contributes to impaired remyelination (Kuhlmann et al., 2008). On the basis of these findings and those in the literature, one may conclude that remyelination is dependent on several factors, one of which is distinct expression patterns of membrane proteins. Remyelination may not only restore saltatory conduction, but also actively assists in preserving axonal integrity. 


\section{REFERENCE LIST}

(1999). TNF neutralization in MS: results of a randomized, placebo-controlled multicenter study. The Lenercept Multiple Sclerosis Study Group and The University of British Columbia MS/MRI Analysis Group. Neurology 53, 457-465.

Arnett,H.A., Mason,J., Marino,M., Suzuki,K., Matsushima,G.K., and Ting,J.P. (2001). TNF alpha promotes proliferation of oligodendrocyte progenitors and remyelination. Nat. Neurosci. 4, 1116-1122.

Arun,T., Tomassini,V., Sbardella,E., de Ruiter,M.B., Matthews,L., Leite,M.I., Gelineau-Morel,R., Cavey,A., Vergo,S., Craner,M., Fugger,L., Rovira,A., Jenkinson,M., and Palace,J. (2013). Targeting ASIC1 in primary progressive multiple sclerosis: evidence of neuroprotection with amiloride. Brain 136, 106-115.

Bacia,A., Wollmann,R., and Soliven,B. (2004). K+ channel blockade impairs remyelination in the cuprizone model. Glia 48, 156-165.

Bakker,D.A., and Ludwin,S.K. (1987). Blood-brain barrier permeability during Cuprizone-induced demyelination. Implications for the pathogenesis of immunemediated demyelinating diseases. J. Neurol. Sci. 78, 125-137.

Bechtold,D.A., Kapoor,R., and Smith,K.J. (2004). Axonal protection using flecainide in experimental autoimmune encephalomyelitis. Ann. Neurol. 55, 607616.

Bechtold,D.A., Miller,S.J., Dawson,A.C., Sun,Y., Kapoor,R., Berry,D., and Smith,K.J. (2006). Axonal protection achieved in a model of multiple sclerosis using lamotrigine. J. Neurol. 253, 1542-1551.

Bechtold,D.A., Yue,X., Evans,R.M., Davies,M., Gregson,N.A., and Smith,K.J. (2005). Axonal protection in experimental autoimmune neuritis by the sodium channel blocking agent flecainide. Brain 128, 18-28.

Benardais,K., Kotsiari,A., Skuljec,J., Koutsoudaki,P.N., Gudi,V., Singh,V., Vulinovic,F., Skripuletz,T., and Stangel,M. (2013). Cuprizone 
[bis(cyclohexylidenehydrazide)] is selectively toxic for mature oligodendrocytes. Neurotox. Res. 24, 244-250.

Benarroch,E.E. (2013). Microglia: Multiple roles in surveillance, circuit shaping, and response to injury. Neurology $81,1079-1088$.

Biancotti,J.C., Kumar,S., and de,V.J. (2008). Activation of inflammatory response by a combination of growth factors in cuprizone-induced demyelinated brain leads to myelin repair. Neurochem. Res. 33, 2615-2628.

Bitsch,A., Schuchardt,J., Bunkowski,S., Kuhlmann,T., and Brück,W. (2000). Acute axonal injury in multiple sclerosis. Correlation with demyelination and inflammation. Brain 123 ( Pt 6), 1174-1183.

Bittner,S., and Meuth,S.G. (2013). Targeting ion channels for the treatment of autoimmune neuroinflammation. Ther. Adv. Neurol. Disord. 6, 322-336.

Bjartmar,C., and Trapp,B.D. (2001). Axonal and neuronal degeneration in multiple sclerosis: mechanisms and functional consequences. Curr. Opin. Neurol. 14, 271278.

Black,J.A., Liu,S., Hains,B.C., Saab,C.Y., and Waxman,S.G. (2006). Long-term protection of central axons with phenytoin in monophasic and chronic-relapsing EAE. Brain 129, 3196-3208.

Black,J.A., Newcombe,J., Trapp,B.D., and Waxman,S.G. (2007). Sodium channel expression within chronic multiple sclerosis plaques. J. Neuropathol. Exp. Neurol. $66,828-837$.

Blakemore,W.F. (1973). Remyelination of the superior cerebellar peduncle in the mouse following demyelination induced by feeding cuprizone. J. Neurol. Sci. 20, 73-83.

Bramlett,H.M., Kraydieh,S., Green,E.J., and Dietrich,W.D. (1997). Temporal and regional patterns of axonal damage following traumatic brain injury: a betaamyloid precursor protein immunocytochemical study in rats. J. Neuropathol. Exp. Neurol. 56, 1132-1141. 
Brown,G.C., and Borutaite,V. (2002). Nitric oxide inhibition of mitochondrial respiration and its role in cell death. Free Radic. Biol. Med. 33, 1440-1450.

Brück,W., Pförtner,R., Pham,T., Zhang,J., Hayardeny,L., Piryatinsky,V., Hanisch,U.K., Regen,T., van,R.D., Brakelmann,L., Hagemeier,K., Kuhlmann,T., Stadelmann,C., John,G.R., Kramann,N., and Wegner,C. (2012). Reduced astrocytic NF-kappaB activation by laquinimod protects from cuprizone-induced demyelination. Acta Neuropathol. 124, 411-424.

Brück,W., Porada,P., Poser,S., Rieckmann,P., Hanefeld,F., Kretzschmar,H.A., and Lassmann,H. (1995). Monocyte/macrophage differentiation in early multiple sclerosis lesions. Ann. Neurol. 38, 788-796.

Brück,W., Schmied,M., Suchanek,G., Brück,Y., Breitschopf,H., Poser,S., Piddlesden,S., and Lassmann,H. (1994). Oligodendrocytes in the early course of multiple sclerosis. Ann. Neurol. 35, 65-73.

Buschmann,J.P., Berger,K., Awad,H., Clarner,T., Beyer,C., and Kipp,M. (2012). Inflammatory response and chemokine expression in the white matter corpus callosum and gray matter cortex region during cuprizone-induced demyelination. J. Mol. Neurosci. 48, 66-76.

Caminero,A., Comabella,M., and Montalban,X. (2011). Tumor necrosis factor alpha (TNF-alpha), anti-TNF-alpha and demyelination revisited: an ongoing story. J. Neuroimmunol. 234, 1-6.

Carlton,W.W. (1967). Studies on the induction of hydrocephalus and spongy degeneration by cuprizone feeding and attempts to antidote the toxicity. Life Sci. 6, 11-19.

Carlton,W.W. (1969). Spongiform encephalopathy induced in rats and guinea pigs by cuprizone. Exp. Mol. Pathol. 10, 274-287.

Charles,P., Reynolds,R., Seilhean,D., Rougon,G., Aigrot,M.S., Niezgoda,A., Zalc,B., and Lubetzki,C. (2002). Re-expression of PSA-NCAM by demyelinated axons: an inhibitor of remyelination in multiple sclerosis? Brain 125, 1972-1979. 
Colombo,E., Cordiglieri,C., Melli,G., Newcombe,J., Krumbholz,M., Parada,L.F., Medico,E., Hohlfeld,R., Meinl,E., and Farina,C. (2012). Stimulation of the neurotrophin receptor TrkB on astrocytes drives nitric oxide production and neurodegeneration. J. Exp. Med. 209, 521-535.

Coman,I., Aigrot,M.S., Seilhean,D., Reynolds,R., Girault,J.A., Zalc,B., and Lubetzki,C. (2006). Nodal, paranodal and juxtaparanodal axonal proteins during demyelination and remyelination in multiple sclerosis. Brain 129, 3186-3195.

Craner,M.J., Hains,B.C., Lo,A.C., Black,J.A., and Waxman,S.G. (2004a). Colocalization of sodium channel Nav1.6 and the sodium-calcium exchanger at sites of axonal injury in the spinal cord in EAE. Brain 127, 294-303.

Craner,M.J., Newcombe,J., Black,J.A., Hartle,C., Cuzner,M.L., and Waxman,S.G. (2004b). Molecular changes in neurons in multiple sclerosis: altered axonal expression of Nav1.2 and Nav1.6 sodium channels and $\mathrm{Na}+/ \mathrm{Ca} 2+$ exchanger. Proc. Natl. Acad. Sci. U. S. A 101, 8168-8173.

Crawford,D.K., Mangiardi,M., Xia,X., Lopez-Valdes,H.E., and Tiwari-Woodruff,S.K. (2009). Functional recovery of callosal axons following demyelination: a critical window. Neuroscience 164, 1407-1421.

Diemel,L.T., Jackson,S.J., and Cuzner,M.L. (2003). Role for TGF-beta1, FGF-2 and PDGF-AA in a myelination of CNS aggregate cultures enriched with macrophages. J. Neurosci. Res. 74, 858-867.

Dikranian,K., Cohen,R., Mac,D.C., Pan,Y., Brakefield,D., Bayly,P., and Parsadanian,A. (2008). Mild traumatic brain injury to the infant mouse causes robust white matter axonal degeneration which precedes apoptotic death of cortical and thalamic neurons. Exp. Neurol. 211, 551-560.

Dupree,J.L., Mason,J.L., Marcus,J.R., Stull,M., Levinson,R., Matsushima,G.K., and Popko,B. (2004). Oligodendrocytes assist in the maintenance of sodium channel clusters independent of the myelin sheath. Neuron Glia Biol. 1, 179-192. 
Dziedzic,T., Metz,I., Dallenga,T., Konig,F.B., Muller,S., Stadelmann,C., and Brück,W. (2010). Wallerian degeneration: a major component of early axonal pathology in multiple sclerosis. Brain Pathol. 20, 976-985.

Eisenbach,M., Kartvelishvily,E., Eshed-Eisenbach,Y., Watkins,T., Sorensen,A., Thomson,C., Ranscht,B., Barnett,S.C., Brophy,P., and Peles,E. (2009). Differential clustering of Caspr by oligodendrocytes and Schwann cells. J. Neurosci. Res. 87, 3492-3501.

Ferguson,B., Matyszak,M.K., Esiri,M.M., and Perry,V.H. (1997). Axonal damage in acute multiple sclerosis lesions. Brain 120 ( Pt 3), 393-399.

Flatmark,T., Kryvi,H., and Tangeras,A. (1980). Induction of megamitochondria by cuprizone(biscyclohexanone oxaldihydrazone). Evidence for an inhibition of the mitochondrial division process. Eur. J. Cell Biol. 23, 141-148.

Foote,A.K., and Blakemore,W.F. (2005a). Inflammation stimulates remyelination in areas of chronic demyelination. Brain 128, 528-539.

Foote,A.K., and Blakemore,W.F. (2005b). Inflammation stimulates remyelination in areas of chronic demyelination. Brain 128, 528-539.

Friese,M.A., Craner,M.J., Etzensperger,R., Vergo,S., Wemmie,J.A., Welsh,M.J., Vincent,A., and Fugger,L. (2007). Acid-sensing ion channel-1 contributes to axonal degeneration in autoimmune inflammation of the central nervous system. Nat. Med. 13, 1483-1489.

Gelfand,J.M. (2014). Multiple sclerosis: diagnosis, differential diagnosis, and clinical presentation. Handb. Clin. Neurol. 122, 269-290.

Gobel,K., Wedell,J.H., Herrmann,A.M., Wachsmuth,L., Pankratz,S., Bittner,S., Budde,T., Kleinschnitz,C., Faber,C., Wiendl,H., and Meuth,S.G. (2013). 4Aminopyridine ameliorates mobility but not disease course in an animal model of multiple sclerosis. Exp. Neurol. 248, 62-71.

Goodin,D.S. (2014a). Glucocorticoid treatment of multiple sclerosis. Handb. Clin. Neurol. 122, 455-464. 
Goodin,D.S. (2014b). The epidemiology of multiple sclerosis: insights to disease pathogenesis. Handb. Clin. Neurol. 122, 231-266.

Gudi,V., Gingele,S., Skripuletz,T., and Stangel,M. (2014). Glial response during cuprizone-induced de- and remyelination in the CNS: lessons learned. Front Cell Neurosci. 8, 73.

Gudi,V., Moharregh-Khiabani,D., Skripuletz,T., Koutsoudaki,P.N., Kotsiari,A., Skuljec,J., Trebst,C., and Stangel,M. (2009). Regional differences between grey and white matter in cuprizone induced demyelination. Brain Res. 1283, 127-138.

Gudi,V., Skuljec,J., Yildiz,O., Frichert,K., Skripuletz,T., Moharregh-Khiabani,D., Voss,E., Wissel,K., Wolter,S., and Stangel,M. (2011). Spatial and temporal profiles of growth factor expression during CNS demyelination reveal the dynamics of repair priming. PLoS. One. 6, e22623.

Hanisch,U.K., and Kettenmann,H. (2007). Microglia: active sensor and versatile effector cells in the normal and pathologic brain. Nat. Neurosci. 10, 1387-1394.

Hayes,K.C. (2004). The use of 4-aminopyridine (fampridine) in demyelinating disorders. CNS. Drug Rev. 10, 295-316.

Herrero-Herranz,E., Pardo,L.A., Bunt,G., Gold,R., Stuhmer,W., and Linker,R.A. (2007). Re-expression of a developmentally restricted potassium channel in autoimmune demyelination: Kv1.4 is implicated in oligodendroglial proliferation. Am. J. Pathol. 171, 589-598.

Herrero-Herranz,E., Pardo,L.A., Gold,R., and Linker,R.A. (2008). Pattern of axonal injury in murine myelin oligodendrocyte glycoprotein induced experimental autoimmune encephalomyelitis: implications for multiple sclerosis. Neurobiol. Dis. 30, 162-173.

Hesse,A., Wagner,M., Held,J., Brück,W., Salinas-Riester,G., Hao,Z., Waisman,A., and Kuhlmann,T. (2010). In toxic demyelination oligodendroglial cell death occurs early and is FAS independent. Neurobiol. Dis. 37, 362-369. 
Hibbits,N., Yoshino,J., Le,T.Q., and Armstrong,R.C. (2012). Astrogliosis during acute and chronic cuprizone demyelination and implications for remyelination. ASN. Neuro. 4, 393-408.

Hickey,W.F., Hsu,B.L., and Kimura,H. (1991). T-lymphocyte entry into the central nervous system. J. Neurosci. Res. 28, 254-260.

Hinks,G.L., and Franklin,R.J. (1999). Distinctive patterns of PDGF-A, FGF-2, IGFI, and TGF-beta1 gene expression during remyelination of experimentally-induced spinal cord demyelination. Mol. Cell Neurosci. 14, 153-168.

Hiremath,M.M., Chen,V.S., Suzuki,K., Ting,J.P., and Matsushima,G.K. (2008). MHC class II exacerbates demyelination in vivo independently of $\mathrm{T}$ cells. $\mathrm{J}$. Neuroimmunol. 203, 23-32.

Hiremath,M.M., Saito,Y., Knapp,G.W., Ting,J.P., Suzuki,K., and Matsushima,G.K. (1998). Microglial/macrophage accumulation during cuprizone-induced demyelination in C57BL/6 mice. J. Neuroimmunol. 92, 38-49.

Huseby,E.S., Liggitt,D., Brabb,T., Schnabel,B., Ohlen,C., and Goverman,J. (2001). A pathogenic role for myelin-specific CD8(+) T cells in a model for multiple sclerosis. J. Exp. Med. 194, 669-676.

Irvine,K.A., and Blakemore,W.F. (2006). Age increases axon loss associated with primary demyelination in cuprizone-induced demyelination in C57BL/6 mice. J. Neuroimmunol. 175, 69-76.

Irvine,K.A., and Blakemore,W.F. (2008). Remyelination protects axons from demyelination-associated axon degeneration. Brain 131, 1464-1477.

Jurevics,H., Largent,C., Hostettler,J., Sammond,D.W., Matsushima,G.K., Kleindienst,A., Toews,A.D., and Morell,P. (2002). Alterations in metabolism and gene expression in brain regions during cuprizone-induced demyelination and remyelination. J. Neurochem. 82, 126-136. 
Kamal,A., Stokin,G.B., Yang,Z., Xia,C.H., and Goldstein,L.S. (2000). Axonal transport of amyloid precursor protein is mediated by direct binding to the kinesin light chain subunit of kinesin-I. Neuron 28, 449-459.

Kapoor,R., Davies,M., Blaker,P.A., Hall,S.M., and Smith,K.J. (2003). Blockers of sodium and calcium entry protect axons from nitric oxide-mediated degeneration. Ann. Neurol. 53, 174-180.

Kesterson,J.W., and Carlton,W.W. (1972). Cuprizone toxicosis in mice--attempts to antidote the toxicity. Toxicol. Appl. Pharmacol. 22, 6-13.

Kettenmann,H., Hanisch,U.K., Noda,M., and Verkhratsky,A. (2011). Physiology of microglia. Physiol Rev. 91, 461-553.

Kettenmann,H., Kirchhoff,F., and Verkhratsky,A. (2013). Microglia: new roles for the synaptic stripper. Neuron 77, 10-18.

Kiryu-Seo,S., Ohno,N., Kidd,G.J., Komuro,H., and Trapp,B.D. (2010). Demyelination increases axonal stationary mitochondrial size and the speed of axonal mitochondrial transport. J. Neurosci. 30, 6658-6666.

KLUVER,H., and BARRERA,E. (1953). A method for the combined staining of cells and fibers in the nervous system. J. Neuropathol. Exp. Neurol. 12, 400-403.

Kondo,A., Nakano,T., and Suzuki,K. (1987). Blood-brain barrier permeability to horseradish peroxidase in twitcher and cuprizone-intoxicated mice. Brain Res. 425, 186-190.

Koo,E.H., Sisodia,S.S., Archer,D.R., Martin,L.J., Weidemann,A., Beyreuther,K., Fischer,P., Masters,C.L., and Price,D.L. (1990). Precursor of amyloid protein in Alzheimer disease undergoes fast anterograde axonal transport. Proc. Natl. Acad. Sci. U. S. A 87, 1561-1565.

Kornek,B., Storch,M.K., Bauer,J., Djamshidian,A., Weissert,R., Wallstroem,E., Stefferl,A., Zimprich,F., Olsson,T., Linington,C., Schmidbauer,M., and Lassmann,H. (2001). Distribution of a calcium channel subunit in dystrophic axons 
in multiple sclerosis and experimental autoimmune encephalomyelitis. Brain 124, 1114-1124.

Kornek,B., Storch,M.K., Weissert,R., Wallstroem,E., Stefferl,A., Olsson,T., Linington,C., Schmidbauer,M., and Lassmann,H. (2000). Multiple sclerosis and chronic autoimmune encephalomyelitis: a comparative quantitative study of axonal injury in active, inactive, and remyelinated lesions. Am. J. Pathol. 157, 267-276.

Korner,H., Lemckert,F.A., Chaudhri,G., Etteldorf,S., and Sedgwick,J.D. (1997). Tumor necrosis factor blockade in actively induced experimental autoimmune encephalomyelitis prevents clinical disease despite activated $\mathrm{T}$ cell infiltration to the central nervous system. Eur. J. Immunol. 27, 1973-1981.

Krauthausen,M., Saxe,S., Zimmermann,J., Emrich,M., Heneka,M.T., and Muller,M. (2014). CXCR3 modulates glial accumulation and activation in cuprizone-induced demyelination of the central nervous system. J. Neuroinflammation. 11, 109.

Kremenchutzky,M., Rice,G.P., Baskerville,J., Wingerchuk,D.M., and Ebers,G.C. (2006). The natural history of multiple sclerosis: a geographically based study 9: observations on the progressive phase of the disease. Brain 129, 584-594.

Kuhlmann,T., Lingfeld,G., Bitsch,A., Schuchardt,J., and Brück,W. (2002). Acute axonal damage in multiple sclerosis is most extensive in early disease stages and decreases over time. Brain 125, 2202-2212.

Kuhlmann,T., Miron,V., Cui,Q., Wegner,C., Antel,J., and Brück,W. (2008). Differentiation block of oligodendroglial progenitor cells as a cause for remyelination failure in chronic multiple sclerosis. Brain 131, 1749-1758.

Kutzelnigg,A., and Lassmann,H. (2014). Pathology of multiple sclerosis and related inflammatory demyelinating diseases. Handb. Clin. Neurol. 122, 15-58.

Kutzelnigg,A., Lucchinetti,C.F., Stadelmann,C., Brück,W., Rauschka,H., Bergmann,M., Schmidbauer,M., Parisi,J.E., and Lassmann,H. (2005). Cortical demyelination and diffuse white matter injury in multiple sclerosis. Brain. 128, 2705-2712. 
Lassmann,H. (2011). Review: the architecture of inflammatory demyelinating lesions: implications for studies on pathogenesis. Neuropathol. Appl. Neurobiol. 37, 698-710.

Lee,D.H., Geyer,E., Flach,A.C., Jung,K., Gold,R., Flugel,A., Linker,R.A., and Luhder,F. (2012). Central nervous system rather than immune cell-derived BDNF mediates axonal protective effects early in autoimmune demyelination. Acta Neuropathol. 123, 247-258.

Lindner,M., Fokuhl,J., Linsmeier,F., Trebst,C., and Stangel,M. (2009). Chronic toxic demyelination in the central nervous system leads to axonal damage despite remyelination. Neurosci. Lett. 453, 120-125.

Lindner,M., Heine,S., Haastert,K., Garde,N., Fokuhl,J., Linsmeier,F., Grothe,C., Baumgartner,W., and Stangel,M. (2008). Sequential myelin protein expression during remyelination reveals fast and efficient repair after central nervous system demyelination. Neuropathol. Appl. Neurobiol. 34, 105-114.

Linker,R.A., Lee,D.H., Demir,S., Wiese,S., Kruse,N., Siglienti,I., Gerhardt,E., Neumann,H., Sendtner,M., Luhder,F., and Gold,R. (2010). Functional role of brain-derived neurotrophic factor in neuroprotective autoimmunity: therapeutic implications in a model of multiple sclerosis. Brain 133, 2248-2263.

Lo,A.C., Saab,C.Y., Black,J.A., and Waxman,S.G. (2003). Phenytoin protects spinal cord axons and preserves axonal conduction and neurological function in a model of neuroinflammation in vivo. J. Neurophysiol. 90, 3566-3571.

Lovett-Racke,A.E., Trotter,J.L., Lauber,J., Perrin,P.J., June,C.H., and Racke,M.K. (1998). Decreased dependence of myelin basic protein-reactive T cells on CD28mediated costimulation in multiple sclerosis patients. A marker of activated/memory T cells. J. Clin. Invest 101, 725-730.

Lubetzki,C., and Stankoff,B. (2014). Demyelination in multiple sclerosis. Handb. Clin. Neurol. 122, 89-99.

Lublin,F.D., and Reingold,S.C. (1996). Defining the clinical course of multiple sclerosis: results of an international survey. National Multiple Sclerosis Society 
(USA) Advisory Committee on Clinical Trials of New Agents in Multiple Sclerosis. Neurology 46, 907-911.

Lucchinetti,C., $\quad$ Brück,W., Parisi,J., $\quad$ Scheithauer,B., Rodriguez,M., and Lassmann,H. (2000). Heterogeneity of multiple sclerosis lesions: implications for the pathogenesis of demyelination. Ann. Neurol. 47, 707-717.

Mahad,D.J., Ziabreva,I., Campbell,G., Lax,N., White,K., Hanson,P.S., Lassmann,H., and Turnbull,D.M. (2009). Mitochondrial changes within axons in multiple sclerosis. Brain 132, 1161-1174.

Manrique-Hoyos,N., Jurgens,T., Gronborg,M., Kreutzfeldt,M., Schedensack,M., Kuhlmann,T., Schrick,C., Brück,W., Urlaub,H., Simons,M., and Merkler,D. (2012). Late motor decline after accomplished remyelination: impact for progressive multiple sclerosis. Ann. Neurol. 71, 227-244.

Mason,J.L., Jones,J.J., Taniike,M., Morell,P., Suzuki,K., and Matsushima,G.K. (2000a). Mature oligodendrocyte apoptosis precedes IGF-1 production and oligodendrocyte progenitor accumulation and differentiation during demyelination/remyelination. J. Neurosci. Res. 61, 251-262.

Mason,J.L., Langaman,C., Morell,P., Suzuki,K., and Matsushima,G.K. (2001a). Episodic demyelination and subsequent remyelination within the murine central nervous system: changes in axonal calibre. Neuropathol. Appl. Neurobiol. 27, 5058.

Mason,J.L., Suzuki,K., Chaplin,D.D., and Matsushima,G.K. (2001b). Interleukin1 beta promotes repair of the CNS. J. Neurosci. 21, 7046-7052.

Mason,J.L., Toews,A., Hostettler,J.D., Morell,P., Suzuki,K., Goldman,J.E., and Matsushima,G.K. (2004). Oligodendrocytes and progenitors become progressively depleted within chronically demyelinated lesions. Am. J. Pathol. 164, 1673-1682.

Mason,J.L., Ye,P., Suzuki,K., D'Ercole,A.J., and Matsushima,G.K. (2000b). Insulin-like growth factor-1 inhibits mature oligodendrocyte apoptosis during primary demyelination. J. Neurosci. 20, 5703-5708. 
Matsushima,G.K., and Morell,P. (2001). The neurotoxicant, cuprizone, as a model to study demyelination and remyelination in the central nervous system. Brain Pathol. 11, 107-116.

Mc,G.C., Beyaert,R., and van,L.G. (2011). Death receptor signalling in central nervous system inflammation and demyelination. Trends Neurosci. 34, 619-628.

McDonald,W.I., Compston,A., Edan,G., Goodkin,D., Hartung,H.P., Lublin,F.D., McFarland,H.F., Paty,D.W., Polman,C.H., Reingold,S.C., Sandberg-Wollheim,M., Sibley,W., Thompson,A., van den Noort,S., Weinshenker,B.Y., and Wolinsky,J.S. (2001). Recommended diagnostic criteria for multiple sclerosis: guidelines from the International Panel on the diagnosis of multiple sclerosis. Ann. Neurol. 50, 121-127.

McKenzie,K.J., McLellan,D.R., Gentleman,S.M., Maxwell,W.L., Gennarelli,T.A., and Graham,D.I. (1996). Is beta-APP a marker of axonal damage in shortsurviving head injury? Acta Neuropathol. 92, 608-613.

McKinnon,R.D., Piras,G., Ida,J.A., Jr., and Dubois-Dalcq,M. (1993). A role for TGF-beta in oligodendrocyte differentiation. J. Cell Biol. 121, 1397-1407.

McMahon,E.J., Suzuki,K., and Matsushima,G.K. (2002). Peripheral macrophage recruitment in cuprizone-induced CNS demyelination despite an intact blood-brain barrier. J. Neuroimmunol. 130, 32-45.

Melief,J., Schuurman,K.G., van de Garde,M.D., Smolders,J., van,E.M., Hamann,J., and Huitinga,I. (2013). Microglia in normal appearing white matter of multiple sclerosis are alerted but immunosuppressed. Glia 61, 1848-1861.

Melzer,N., and Meuth,S.G. (2014). Disease-modifying therapy in multiple sclerosis and chronic inflammatory demyelinating polyradiculoneuropathy: common and divergent current and future strategies. Clin. Exp. Immunol. 175, 359-372.

Mildner,A., Schmidt,H., Nitsche,M., Merkler,D., Hanisch,U.K., Mack,M., Heikenwalder,M., Brück,W., Priller,J., and Prinz,M. (2007). Microglia in the adult brain arise from Ly-6ChiCCR2+ monocytes only under defined host conditions. Nat. Neurosci. 10, 1544-1553. 
Millecamps,S., and Julien,J.P. (2013). Axonal transport deficits and neurodegenerative diseases. Nat. Rev. Neurosci. 14, 161-176.

Murray,P.D., McGavern,D.B., Sathornsumetee,S., and Rodriguez,M. (2001). Spontaneous remyelination following extensive demyelination is associated with improved neurological function in a viral model of multiple sclerosis. Brain 124, 1403-1416.

Nikic,I., Merkler,D., Sorbara,C., Brinkoetter,M., Kreutzfeldt,M., Bareyre,F.M., Brück,W., Bishop,D., Misgeld,T., and Kerschensteiner,M. (2011). A reversible form of axon damage in experimental autoimmune encephalomyelitis and multiple sclerosis. Nat. Med. 17, 495-499.

Otsuka,N., Tomonaga,M., and Ikeda,K. (1991). Rapid appearance of beta-amyloid precursor protein immunoreactivity in damaged axons and reactive glial cells in rat brain following needle stab injury. Brain Res. 568, 335-338.

Patel,J.R., McCandless,E.E., Dorsey,D., and Klein,R.S. (2010). CXCR4 promotes differentiation of oligodendrocyte progenitors and remyelination. Proc. Natl. Acad. Sci. U. S. A 107, 11062-11067.

Pattison,I.H., and Jebbett,J.N. (1971a). Clinical and histological observations on cuprizone toxicity and scrapie in mice. Res. Vet. Sci. 12, 378-380.

Pattison,I.H., and Jebbett,J.N. (1971b). Histopathological similarities between scrapie and cuprizone toxicity in mice. Nature 230, 115-117.

Pierce,J.E., Trojanowski,J.Q., Graham,D.I., Smith,D.H., and Mclntosh,T.K. (1996). Immunohistochemical characterization of alterations in the distribution of amyloid precursor proteins and beta-amyloid peptide after experimental brain injury in the rat. J. Neurosci. 16, 1083-1090.

Polman,C.H., Reingold,S.C., Banwell,B., Clanet,M., Cohen,J.A., Filippi,M., Fujihara,K., Havrdova,E., Hutchinson,M., Kappos,L., Lublin,F.D., Montalban,X., O'Connor,P., $\quad$ Sandberg-Wollheim,M., Thompson,A.J., $\quad$ Waubant,E., Weinshenker,B., and Wolinsky,J.S. (2011). Diagnostic criteria for multiple sclerosis: 2010 revisions to the McDonald criteria. Ann. Neurol. 69, 292-302. 
Remington,L.T., Babcock,A.A., Zehntner,S.P., and Owens,T. (2007). Microglial recruitment, activation, and proliferation in response to primary demyelination. Am. J. Pathol. 170, 1713-1724.

RICHARDSON,K.C., JARETT,L., and FINKE,E.H. (1960). Embedding in epoxy resins for ultrathin sectioning in electron microscopy. Stain Technol. 35, 313-323.

Ruddle,N.H., Bergman,C.M., McGrath,K.M., Lingenheld,E.G., Grunnet,M.L., Padula,S.J., and Clark,R.B. (1990). An antibody to lymphotoxin and tumor necrosis factor prevents transfer of experimental allergic encephalomyelitis. J. Exp. Med. 172, 1193-1200.

Schattling,B., Eggert,B., and Friese,M.A. (2014). Acquired channelopathies as contributors to development and progression of multiple sclerosis. Exp. Neurol.

Schluesener,H.J., and Wekerle,H. (1985). Autoaggressive T lymphocyte lines recognizing the encephalitogenic region of myelin basic protein: in vitro selection from unprimed rat T lymphocyte populations. J. Immunol. 135, 3128-3133.

Schmidt,K., Eulitz,D., Veh,R.W., Kettenmann,H., and Kirchhoff,F. (1999). Heterogeneous expression of voltage-gated potassium channels of the shaker family (Kv1) in oligodendrocyte progenitors. Brain Res. 843, 145-160.

Scholz,C., Patton,K.T., Anderson,D.E., Freeman,G.J., and Hafler,D.A. (1998). Expansion of autoreactive $T$ cells in multiple sclerosis is independent of exogenous B7 costimulation. J. Immunol. 160, 1532-1538.

Schuh,C., Wimmer,I., Hametner,S., Haider,L., Van Dam,A.M., Liblau,R.S., Smith,K.J., Probert,L., Binder,C.J., Bauer,J., Bradl,M., Mahad,D., and Lassmann,H. (2014). Oxidative tissue injury in multiple sclerosis is only partly reflected in experimental disease models. Acta Neuropathol. 128, 247-266.

Sedgwick,J.D., and Mason,D.W. (1986). The mechanism of inhibition of experimental allergic encephalomyelitis in the rat by monoclonal antibody against CD4. J. Neuroimmunol. 13, 217-232. 
Selmaj,K., Raine,C.S., and Cross,A.H. (1991). Anti-tumor necrosis factor therapy abrogates autoimmune demyelination. Ann. Neurol. 30, 694-700.

Sherman,D.L., and Brophy,P.J. (2005). Mechanisms of axon ensheathment and myelin growth. Nat. Rev. Neurosci. 6, 683-690.

Simons,M., and Trajkovic,K. (2006). Neuron-glia communication in the control of oligodendrocyte function and myelin biogenesis. J. Cell Sci. 119, 4381-4389.

Skripuletz,T., Gudi,V., Hackstette,D., and Stangel,M. (2011). De- and remyelination in the CNS white and grey matter induced by cuprizone: the old, the new, and the unexpected. Histol. Histopathol. 26, 1585-1597.

Skripuletz,T., Hackstette,D., Bauer,K., Gudi,V., Pul,R., Voss,E., Berger,K., Kipp,M., Baumgartner,W., and Stangel,M. (2013). Astrocytes regulate myelin clearance through recruitment of microglia during cuprizone-induced demyelination. Brain 136, 147-167.

Smith,K.J. (2006). Axonal protection in multiple sclerosis--a particular need during remyelination? Brain 129, 3147-3149.

Smith,K.J. (2007). Sodium channels and multiple sclerosis: roles in symptom production, damage and therapy. Brain Pathol. 17, 230-242.

Smith,K.J., Kapoor,R., Hall,S.M., and Davies,M. (2001). Electrically active axons degenerate when exposed to nitric oxide. Ann. Neurol. 49, 470-476.

Smith,K.J., and Lassmann,H. (2002). The role of nitric oxide in multiple sclerosis. Lancet Neurol. 1, 232-241.

Sofroniew,M.V. (2009). Molecular dissection of reactive astrogliosis and glial scar formation. Trends Neurosci. 32, 638-647.

Sofroniew,M.V., and Vinters,H.V. (2010). Astrocytes: biology and pathology. Acta Neuropathol. 119, 7-35.

Stadelmann,C., Kerschensteiner,M., Misgeld,T., Brück,W., Hohlfeld,R., and Lassmann,H. (2002). BDNF and gp145trkB in multiple sclerosis brain lesions: 
neuroprotective interactions between immune and neuronal cells? Brain 125, 7585.

Stidworthy,M.F., Genoud,S., Suter,U., Mantei,N., and Franklin,R.J. (2003). Quantifying the early stages of remyelination following cuprizone-induced demyelination. Brain Pathol. 13, 329-339.

Suvannavejh,G.C., Lee,H.O., Padilla,J., Dal Canto,M.C., Barrett,T.A., and Miller,S.D. (2000). Divergent roles for p55 and p75 tumor necrosis factor receptors in the pathogenesis of MOG(35-55)-induced experimental autoimmune encephalomyelitis. Cell Immunol. 205, 24-33.

Suzuki,K. (1969). Giant hepatic mitochondria: production in mice fed with cuprizone. Science 163, 81-82.

Taylor,L.C., Gilmore,W., and Matsushima,G.K. (2009). SJL mice exposed to cuprizone intoxication reveal strain and gender pattern differences in demyelination. Brain Pathol. 19, 467-479.

Taylor,L.C., Gilmore,W., Ting,J.P., and Matsushima,G.K. (2010). Cuprizone induces similar demyelination in male and female C57BL/6 mice and results in disruption of the estrous cycle. J. Neurosci. Res. 88, 391-402.

Tiwari-Woodruff,S., Beltran-Parrazal,L., Charles,A., Keck,T., Vu,T., and Bronstein,J. (2006). K+ channel KV3.1 associates with OSP/claudin-11 and regulates oligodendrocyte development. Am. J. Physiol Cell Physiol 291, C687c698.

Toosy,A., Ciccarelli,O., and Thompson,A. (2014). Symptomatic treatment and management of multiple sclerosis. Handb. Clin. Neurol. 122, 513-562.

Trapp,B.D., Peterson,J., Ransohoff,R.M., Rudick,R., Mork,S., and Bo,L. (1998). Axonal transection in the lesions of multiple sclerosis. N. Engl. J. Med. 338, 278285.

Trapp,B.D., and Stys,P.K. (2009). Virtual hypoxia and chronic necrosis of demyelinated axons in multiple sclerosis. Lancet Neurol. 8, 280-291. 
van Oosten,B.W., Barkhof,F., Truyen,L., Boringa,J.B., Bertelsmann,F.W., von Blomberg,B.M., Woody,J.N., Hartung,H.P., and Polman,C.H. (1996). Increased MRI activity and immune activation in two multiple sclerosis patients treated with the monoclonal anti-tumor necrosis factor antibody cA2. Neurology 47, 15311534.

Vergo,S., Craner,M.J., Etzensperger,R., Attfield,K., Friese,M.A., Newcombe,J., Esiri,M., and Fugger,L. (2011). Acid-sensing ion channel 1 is involved in both axonal injury and demyelination in multiple sclerosis and its animal model. Brain $134,571-584$.

Voss,E.V., Skuljec,J., Gudi,V., Skripuletz,T., Pul,R., Trebst,C., and Stangel,M. (2012). Characterisation of microglia during de- and remyelination: can they create a repair promoting environment? Neurobiol. Dis. 45, 519-528.

Westland,K.W., Pollard,J.D., Sander,S., Bonner,J.G., Linington,C., and McLeod,J.G. (1999). Activated non-neural specific T cells open the blood-brain barrier to circulating antibodies. Brain 122 ( Pt 7), 1283-1291.

Witte,M.E., Mahad,D.J., Lassmann,H., and van,H.J. (2014). Mitochondrial dysfunction contributes to neurodegeneration in multiple sclerosis. Trends Mol. Med. 20, 179-187.

Wolswijk,G., and Balesar,R. (2003). Changes in the expression and localization of the paranodal protein Caspr on axons in chronic multiple sclerosis. Brain 126, 1638-1649.

Zambonin,J.L., Zhao,C., Ohno,N., Campbell,G.R., Engeham,S., Ziabreva,I., Schwarz,N., Lee,S.E., Frischer,J.M., Turnbull,D.M., Trapp,B.D., Lassmann,H., Franklin,R.J., and Mahad,D.J. (2011). Increased mitochondrial content in remyelinated axons: implications for multiple sclerosis. Brain 134, 1901-1913.

Zoupi,L., Markoullis,K., Kleopa,K.A., and Karagogeos,D. (2013). Alterations of juxtaparanodal domains in two rodent models of CNS demyelination. Glia 61, 1236-1249. 


\section{CURRICULUM VITAE}

\section{PERSONAL DATA}

Name, Surname:

Verena Schultz

Date of birth:

11.09.1985

Place of birth: Wilhelmshaven

Address: $\quad$ Ginsterweg 4

D-37077 Göttingen

Germany

Nationality: German

\section{EDUCATION AND RESEARCH EXPERIENCE}

08/1998 - 06/2005 Käthe-Kollwitz-Gymnasium

Wilhelmshaven

Certificate: Abitur

10/2005 - 10/2010 Eberhard Karls University of Tübingen

Studies in biochemistry,

Degree: Diplom-Biochemikerin

04/2010 - 10/2010 Diploma thesis in the research group of Dr. H. Kalbacher, IFIB (Interfaculty Institute of Biochemistry, Eberhard Karls University of Tübingen)

„Citrullination of proteins: a diagnostic marker for autoimmune diseases?"

05/2011 - 11/2014 PhD thesis in the research group of Dr. A. Junker, GeorgAugust-University of Göttingen

"Axonal degeneration and protection during early remyelination in multiple sclerosis and an animal model"

\section{EMPLOYMENT HISTORY}

05/2009-12/2009 Student teaching assistant in the research group of Prof. G. Dodt, IFIB

Assistance of the practical course "Cellbiochemistry" for students of biochemistry in the $5^{\text {th }}$ and $6^{\text {th }}$ semester 
11/2010-03/2011 Scientific assistant in the research group of Dr. C. Neufert, Medical Clinic 1, Centre for molecular research of inflammation and cancer, Friedrich-Alexander-University of ErlangenNürnberg

„Role of Th17 cells in inflammatory bowel diseases“

05/2011 - 11/2014 Scientific assistant in the research group of Dr. A. Junker, Georg-August-University of Göttingen

"Axonal degeneration and protection during early remyelination in multiple sclerosis and an animal model"

\section{PUBLICATIONS}

\section{Original articles}

Lescher J, Paap F, Schultz V, Redenbach L, Scheidt U, Rosewich H, Nessler S, Fuchs E, Gärtner J, Brück W, Junker A. (2012) MicroRNA regulation in experimental autoimmune encephalomyelitis in mice and marmosets resembles regulation in human multiple sclerosis lesions. J. Neuroimmunol. 246, 27-33.

\section{Abstracts/ Presentations}

Schultz V, Paap F, Scheidt U, Brück W, Junker A. Axonal degeneration and protection during early remyelination in multiple sclerosis and an animal model. Presented as a poster at the $58^{\text {th }}$ Annual meeting of the German Society for Neuropathology and Neuroanatomy (DGNN), $11^{\text {th }}-14^{\text {th }}$ September 2012, in Göttingen, Germany

Schultz V, Paap F, Scheidt U, Junker A. Disrupted CD47 signaling in MS lesions leads to failed remyelination. Presented as a talk at the $5^{\text {th }}$ NEUROWIND e.V. meeting, $25^{\text {th }}-27^{\text {th }}$ October 2013, in Berlin, Germany

Schultz V, Paap F, Scheidt U, Stadelmann C, Brück W, Junker A. Axonal degeneration and protection during early remyelination in multiple sclerosis and an animal model. Presented as a poster at the $12^{\text {th }}$ International Congress of Neuroimmunology (ISNI), $09^{\text {th }}-13^{\text {th }}$ November 2014 , in Mainz, Germany 